\title{
Ploidy-dependent effect of prolonged photoperiod on mode of reproduction and photosynthesis in the Ranunculus auricomus complex (Ranunculaceae)
}

\author{
Dissertation \\ For the award of degree \\ "Doctor rerum naturalium" \\ at the Georg-August-Universität Göttingen \\ Within the doctoral program Biology \\ of the Georg-August University School of Science (GAUSS)
}

Submitted by

Fuad Bahrul Ulum

From Indonesia

Göttingen, 2021 


\section{Thesis committee}

Prof. Dr. Elvira Hörandl

Department of Systematics, Biodiversity and Evolution of Plants

Albrecht-von-Haller Institute for Plant Sciences

University of Goettingen, Germany

Prof. Dr. Hermann Behling

Department of Palynology and Climate Dynamic

Albrecht-von-Haller Institute for Plant Sciences

University of Goettingen, Germany

\section{Members of the Examination Board}

$1^{\text {st }}$ Reviewer Prof. Dr. Elvira Hörandl

Department of Systematics, Biodiversity and Evolution of Plants

Albrecht-von-Haller Institute for Plant Sciences

University of Goettingen, Germany

$2^{\text {nd }}$ Reviewer Prof. Dr. Hermann Behling

Department of Palynology and Climate Dynamic

Albrecht-von-Haller Institute for Plant Sciences

University of Goettingen, Germany

\section{Further members of the Examination Board}

PD Dr. Franz Hadacek

Dept. of Plant Biochemistry

University of Goettingen, Germany

Jun.-Prof. Dr. Jan de Vries

Institute for Microbiology and Genetics

Department of Applied Bioinformatics

University of Goettingen, Germany

Prof. Dr. Stefan Scheu

J.F. Blumenbach Institute of Zoology and Anthropology

University of Goettingen, Germany

Prof. Dr. Holger Kreft

Department of Biodiversity, Macroecology \& Conservation Biogeography Group

Faculty of Forest Sciences and Forest Ecology

University of Goettingen, Germany

Date of the oral examination: 12 July 2021 


\section{Table of content}

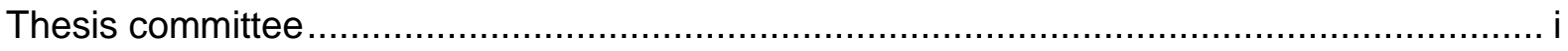

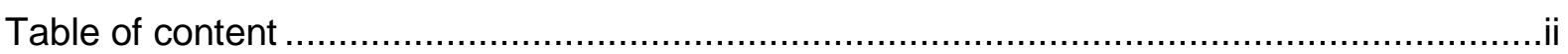

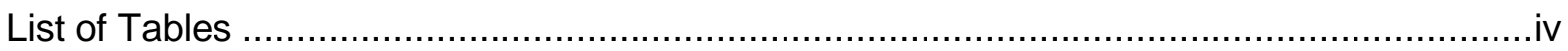

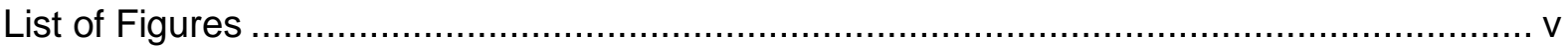

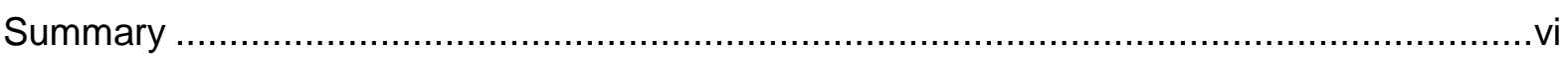

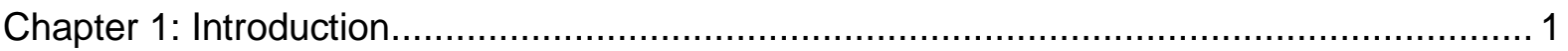

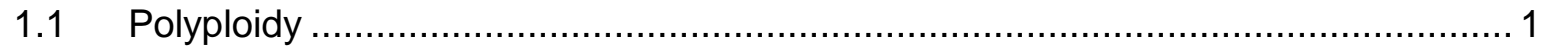

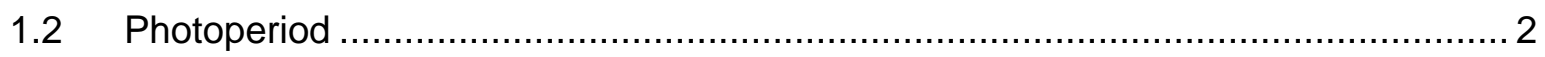

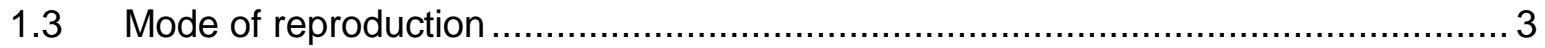

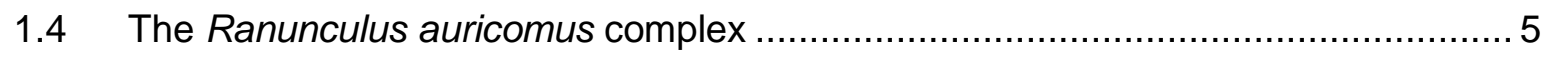

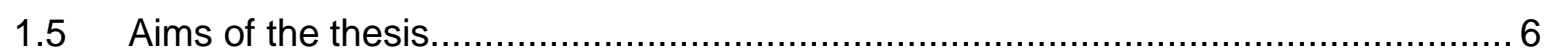

Chapter 2: Ploidy-dependent effects of light stress on the mode of reproduction in the Ranunculus auricomus complex (Ranunculaceae).........................................................

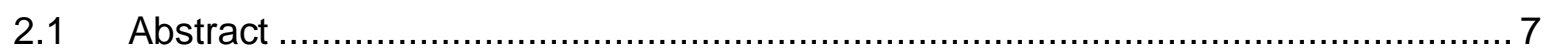

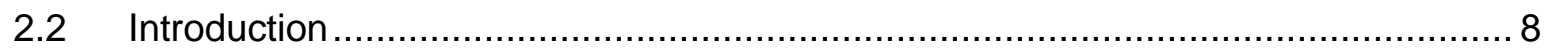

2.3 Materials and Methods..................................................................................... 10

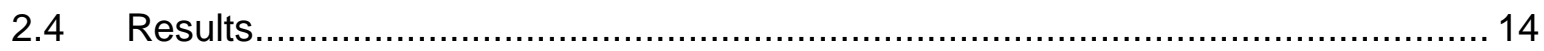

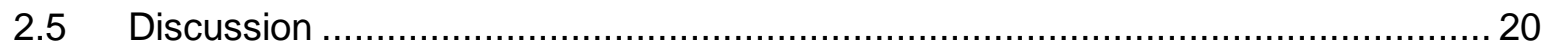

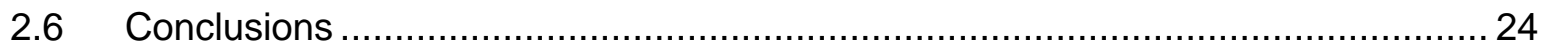

Chapter 3: Ploidy-dependent effects of prolonged photoperiod on photosynthesis performance of the Ranunculus auricomus complex (Ranunculaceae) ................................................ 40

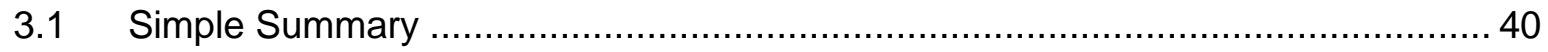

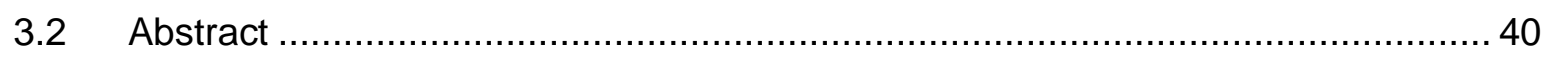

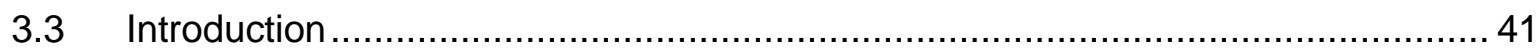

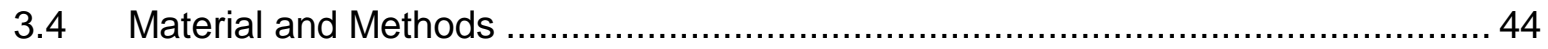

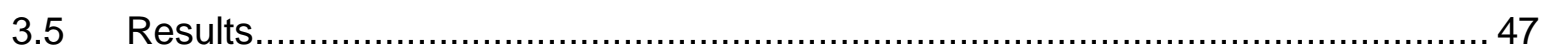

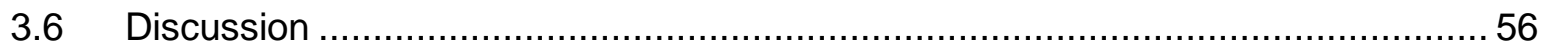

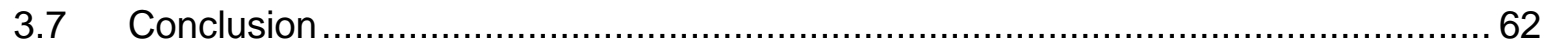




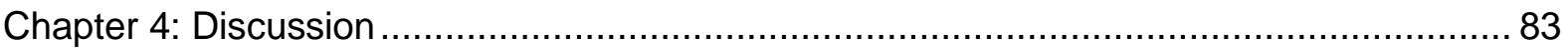

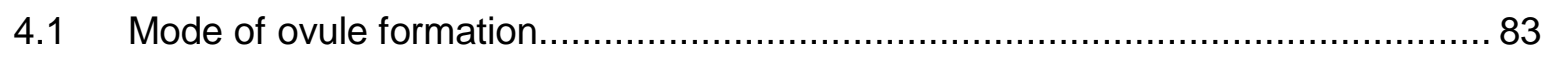

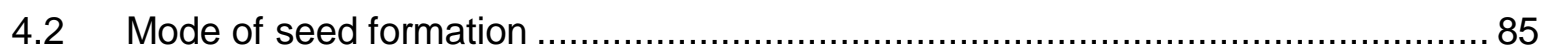

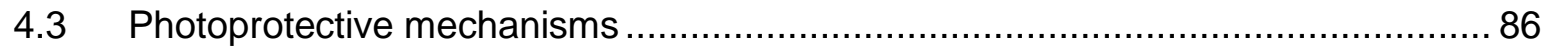

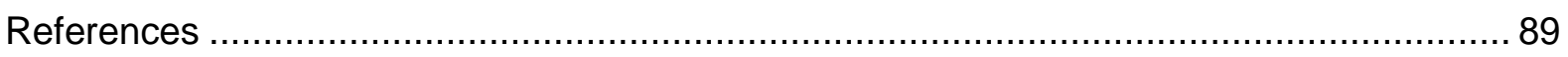

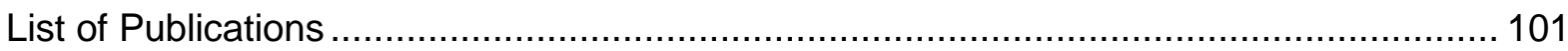

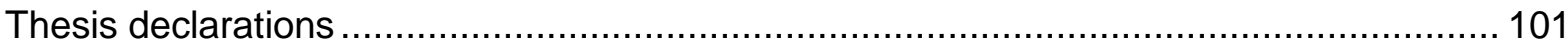

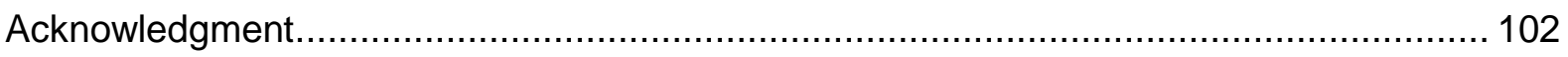

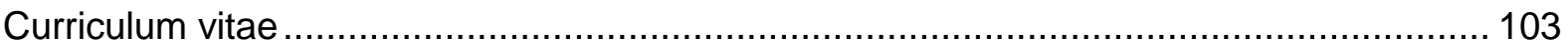




\section{List of Tables}

Table 2.1 P-values for the two way ANOVAs to determine the interaction effect of stress treatment and ploidy level on the proportion of sexual ovules. 16

Table 2.2 Observed reproductive pathways of three cytotypes of the $R$. auricomus complex. 19

Table 3.1 Summary statistics and P- values of Wilcoxon-Mann-Whitney-Test for determination effects of prolonged photoperiod on relative electron transport rate 52 Table 3.2 $P$-values of Kruskal-Wallis-Tests for determination of significant differences of relative electron transport rate, induction curve parameters, and specific energy fluxes parameters of JIP-test. 53

Table 3.3 Summary statistic and $P$ - values of Wilcoxon-Mann-Whitney-Test for determination of effects of prolonged photoperiod on induction curves parameters between treatments... 55 Table 3.4 Summary statistics and $P$ - values of Wilcoxon-Mann-Whitney-Test for determination of effects of prolonged photoperiod on specific energy fluxes parameters of JIP-test between treatments. 59 


\section{List of Figures}

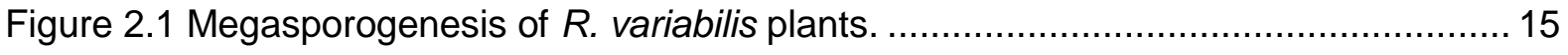

Figure 2.2 Proportions of sexual ovules in the $R$. auricomus complex............................. 15

Figure 2.3 Proportions of well-developed seeds in the $R$. auricomus complex ................... 17

Figure 2.4 Proportions of sexual seeds in the $R$. auricomus complex.............................. 18

Figure 3.1 Photosynthesis performance of Ranunculus auricomus complex ....................... 49

Figure 3.2 Effect photoperiod on the relative electron transport rate, induction curves, and fast

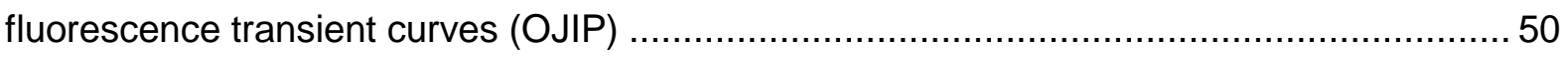

Figure 3.3 Photoperiod effect on relative electron transport rate ................................... 51

Figure 3.4 Photoperiod effect on induction curve parameters....................................... 54

Figure 3.5 Photoperiod effect on specific energy fluxes parameters of JIP-test..................57 


\section{Summary}

Polyploidy, whole-genome duplication, enhances stress-tolerance to drastic environmental compared to their diploid progenitor by enabling more extensive adaptation as advantages of gene and genome duplication. Polyploidy acts as drivers of evolution and speciation in plants. Polyploidy in angiosperms is an influential factor to trigger apomixis, the reproduction of asexual seeds. Apomixis is usually facultative, which means that both sexual and apomictic seeds can be formed by the same plant. Environmental abiotic stress, e.g., light stress, can change the frequency of apomixis. Photoperiod stress in plants influences flowering, photosynthesis, growth, metabolite profiles, and production of reactive oxygen species (ROS). The light stress creates photodamage due to the inhibition of photosystem II (PSII) repair and alternation in the photosynthetic redox signaling pathways. Apomeiosis, the production of unreduced embryo sacs, versus meiotic development is influenced by ROS scavenging. The excess of ROS in reproductive tissue generates oxidative stress. In the archespor, oxidative stress might lead to DNA double-strand breaks (DSBs) and induction of meiosis as a DNA repair mechanism. Stress-adapted plants are able to maintain the metabolic network in ROS scavenging, including compatible solutes, antioxidants, and stress-responsive proteins. In polyploid plants, the higher stress tolerance reduces oxidative stress. Hence, in facultative apomictic polyploids, lowered stress levels could result in a decrease in proportions of meiotic ovules and favor apomeiotic development. The main aims of this research were to explore with prolonged photoperiods whether polyploidy alters proportions of sexual ovule and sexual seed formation under light stress conditions and to observed the extent of stress effect on photosynthesis in the leaves that appear together with the flower buds. I used three facultative apomictic, pseudogamous cytotypes of the Ranunculus auricomus complex (diploid, tetraploid, and hexaploid). Stress treatments were applied by extended light periods $(16.5 \mathrm{~h})$ and control $(10 \mathrm{~h})$ in climate growth chambers. Proportions of apomeiotic vs. meiotic development in the ovule were evaluated with clearing methods, and the mode of seed formation was examined by single seed flow cytometric seed screening (ssFCSS). I further studied pollen stainability to understand the effects of pollen quality on seed formation. In basal leaves, I analyzed the effect of extended photoperiod on photosynthesis efficiency as a proxy of stress conditions. The flower buds are covered by green sepals as photosynthetic tissue, and hence we expect the same photosynthetic performance and stress effects as in the basal leaves. Photosynthesis performance was measured by applying an extensive analysis of chlorophyll a fluorescence to record the parameters: PSII maximum efficiency ( $\phi P S I I)$, the maximum quantum efficiency of PSII photochemistry (QY_max), relative electron transport rate (rETR), fluorescence induction curve (IC) of non-photochemical quenching (NPQ), and fast fluorescence transient curve (OJIP curve). Results revealed that under 
extended photoperiod, all cytotypes produced significantly more sexual ovules than in the controls, with the strongest effects on diploids. The stress treatment affected neither the frequency of seed set nor the proportion of sexual seeds nor pollen quality. Prolonged photoperiod did not enhance the photosynthesis efficiency (QY_max and $\phi P S I I)$ of three cytotypes of $R$. auricomus. Among cytotypes, diploids were the most sensitive to the extended photoperiod compared to polyploids as indicated by the alternation of non-photochemical quenching parameters (NPQ, $q_{E}, N P Q_{E}$, and $q N$ ), specific energy flux parameters ( $A B S / R C$, $\mathrm{DI}_{0} / \mathrm{RC}$, and $\mathrm{TR} / \mathrm{RC}$ ), and performance index on absorption basis (PI_Abs). In tetraploids, the fraction of light excess was quenched into photochemistry $(q P)$, but another fraction exceeded the capacity of photon trapping $\left(\mathrm{TR}_{0} / \mathrm{RC}\right)$, hence dissipated as non-photochemical quenching $(q L)$. The hexaploids presented a variation of photosynthesis performance among two clones which might relate to different habitats. These findings confirm our hypothesis that megasporogenesis is triggered by light stress treatments. Comparisons of cytotypes support the hypothesis that ovule development in polyploid plants is less sensitive to prolonged photoperiods and responds to a lesser extent with sexual ovule formation. Polyploids may better buffer environmental stress, which releases the potential for aposporous ovule development from somatic cells, and may facilitate the establishment of apomictic seed formation. The photosynthesis performance of $R$. auricomus relates to the mode of ovule formation, as diploids showed the highest sensitivity to prolonged photoperiod concomitant to the highest proportions of sexual ovules, followed by tetraploids. Hexaploids, however, exhibited a very large variance in the proportions of sexual ovules, which we also observed here in photosynthesis performance. I suppose that this variation is mostly referable to two different ecotypes. 


\section{Chapter 1: Introduction}

\subsection{Polyploidy}

Polyploidy, the presence of more than two complete sets of chromosomes, is regarded as an extensive feature of chromosome evolution in many eukaryote taxa (Ramsey and Schemske, 1998; Soltis and Soltis, 1999; Comai, 2005; Otto, 2007; Van de Peer et al., 2020). In angiosperms, more than $30 \%$ have one ancient polyploidization event in their history (Masterson, 1994; Landis et al., 2018; Van de Peer et al., 2020). Polyploids arise through hybridization of two species with associated genome doubling (allopolyploidy) or genome doubling in a single species (autopolyploidy) (Grant, 1981; Soltis and Soltis, 2000; Comai, 2005; Landis et al., 2018). Polyploidy provides at least three advantages, i.e., heterosis or hybrid vigor that display polyploids is more vigorous than diploids; gene redundancy protects polyploids from the deleterious effect of mutations; and asexual reproduction facilitates reproduction in the absence of sexual mates. In other words, polyploidy also inhibits several disadvantages, e.g., disruption effect of nuclear and cell enlargement, presence of aneuploidy, and epigenetic instability (Comai, 2005).

In a stable environment, organisms perform slow optimization over time and polyploid individuals may be less competitive to their diploid progenitors, but in a changing environment, polyploids show a better mechanism for more extensive change (Van de Peer et al., 2020). Several studies reported the better adaptive potential of polyploids than diploids to extreme conditions as consequence of higher genetic variation and buffering effect of their duplicate genes in short-term adaptation (Van de Peer et al., 2009; Van de Peer et al., 2017; Doyle and Coate, 2019) and in long-term adaptation (Van de Peer et al., 2020). Polyploid plants are considered to be a common mode of speciation with expanded ecological niches (Baniaga et al., 2020; Van de Peer et al., 2020) and greater colonizing ability over latitudinal and longitudinal gradients (Brochmann et al., 2004; Schinkel et al., 2016; Rice et al., 2019). Polyploids exhibit improvement such as adaptivity to abiotic stress, e.g. drought (del Pozo and Ramirez-Parra, 2014; Martínez et al., 2018), cold (Klatt et al., 2018), heat (Godfree et al., 2017), salt (Chao et al., 2013), and light (Coate et al., 2013), biotic-stress tolerance e.g., against pathogen (Keane et al., 2014; Hannweg et al., 2016; Hias et al., 2017), against competitors (Wu et al., 2019; Harms et al., 2020), in mutualistic interaction photosynthesis efficiency (Anneberg and Segraves, 2019; Acuña-Rodríguez et al., 2020), metabolite alternation (lannicelli et al., 2020), and reactive oxygen species (ROS) scavenging (Deng et al., 2012; Wei et al., 2019). Polyploidy also influences plant reproduction and fitness, especially apomixis, i.e., the asexual reproduction via seed (Asker and Jerling, 1992). The 
establishment of apomixis correlated to the polyploidy has been presented in several studies (Quarin, 1986; Zappacosta et al., 2014; Delgado et al., 2016).

\subsection{Photoperiod}

Photoperiod is the total amount of daily light and darkness exposure to organisms, naturally promoted by the tilt of the earth's axis (Jackson, 2009). In a plant, photoperiod alternation influences light signal in the leaves, circadian rhythm synchronization, bud set, flowering, and vegetative development (Jeong and Clark, 2005; Jackson, 2009), photosynthesis (Bauerle et al., 2012; Kinoshita et al., 2020), growth (Wu et al., 2004), metabolite (Sulpice et al., 2014; de Castro et al., 2019), and mode of reproduction (Saran and de Wet, 1976; Quarin, 1986; Klatt et al., 2016). Based on the plants preference to the daily light period, Major (1980) and Thomas and Vince-Prue (1984) in (Mungoma, 1988) classified plants into three categories:

1. Short-day plants: flowering occurs only in shorter day-lengths than the critical daylength.

2. Long-day plants: flowering is initiated only in day-lengths that exceed the critical daylength.

3. Day-neutral plans: flowering is not affected by day-length.

Among plant structure, the green leaf is the most affected organ by photoperiod stress (Wu et al., 2004) which is associated with the photosynthesis function (Bauerle et al., 2012). Prolongation of the light period induces photoperiod stress and causes damage in photosystem II and resulting photoinhibition (Roeber et al., 2021). Photoperiod stress leads to the accumulation of oxidative stress during the dark period of following extended daylight. On the next day, the stress reduced the photosynthesis performance (Abuelsoud et al., 2020). The enhancement of ROS later turns to block the synthesis of PSII protein in chloroplast (Takahashi and Murata, 2008). Photosynthesis organisms regulate photoprotection mechanisms to avoid net photoinhibition by the movement of leaves and chloroplasts, screening photo radiation, ROS scavenging, dissipation light energy into heat $(q E)$, cyclic electron flow (CEF) around photosystem I (PSI), and photorespiratory pathway (Takahashi and Badger, 2011).

\subsubsection{Stress effect on photosynthesis performance}

Chlorophyll within leaf exists as pigment-protein complexes in PSII, PSI, and within the lightharvesting complexes (LHC) associated with each of these reaction centers (Murchie and Lawson, 2013). During photosynthesis, light energy is absorbed by a light-harvesting complex of PSII and induces a photochemical process in which an electron is transferred from the reaction center chlorophyll, P680, to the primary quinone acceptor of PSII, $Q_{A}$. However, some 
absorbed light energy can be lost from PSII as chlorophyll fluorescence or dissipated into heat. These three fates of absorbed light are in direct competition for excitation energy. If the rate of one direction increases the rate of the other two will decrease (Kautsky and Hirsch, 1931; Maxwell and Johnson, 2000; Baker, 2008). Chlorophyll a fluorescence analysis is widely used method for measurement of photosynthesis performance and represents the plant response to the environmental stress (Krause and Weis, 1991; Maxwell and Johnson, 2000; Müller et al., 2001; Strasser et al., 2004; Ralph and Gademann, 2005; Baker, 2008; Roháček et al., 2008; Murchie and Lawson, 2013; Stirbet et al., 2018). Thus the yield of chlorophyll fluorescence emission gives information about the quantum efficiency of photochemistry and heat dissipation (Murchie and Lawson, 2013). Based on the Chl a fluorescence, we can extract several parameters, i.e., PSII maximum efficiency ( $\phi P S I)$, the maximum quantum efficiency of PSII photochemistry (QY_max), relative electron transport rate ( $r E T R)$, fluorescence induction curve (IC) of non-photochemical quenching (NPQ), and fast fluorescence transient curve (OJIP curve). QY_max gives a robust indicator of the maximum quantum efficiency of PSII photochemistry, and the value of the non-stressed plant is remarkably consistent at ca 0.83 (Björkman and Demmig, 1987). фPSIl gives the proportion of absorbed light that is actually used in PSII. Any decrease in this parameter is reflecting an increase in NPQ. The NPQ estimates heat dissipation and can be separated into higher energy state quenching $q_{E}$, quenching caused by state transitions $\left(q_{T}\right)$ which refer to the migration of peripheral LHCIls from PSII to PSI, and photoinhibition quenching $\left(q_{l}\right)$ that important in high light level and refers to any sustained quenching (Murchie and Lawson, 2013). The OJIP curve shows polyphasic rise as a proxy for the QY_max. Several performance indices from JIP test provide measures of efficiencies of specific electron transport reaction in the thylakoid membrane and can be used to quantify the stress tolerance of the plant (Stirbet et al., 2018).

\subsection{Mode of reproduction}

Reproduction in eukaryotes is the original process of "recapitulation of ontogeny". The reproduction begins with the formation of a zygote that involves syngamy (sexual reproduction) or development from an egg cell without syngamy (asexual reproduction) (Mogie, 1992). These modes of reproduction provide distinct advantages in the natural population. Sexual reproduction in plants serves genetic and phenotypic variation for better adaptation to environmental changes and the breeding of new varieties. In contrast, asexual reproduction yields clonal offspring that are genetically identical to the mother plant, thus fixing complex genotypes (Schmidt et al., 2015). In flowering plans, sexual reproduction is the most common mode of reproduction (Koltunow, 1993; Tucker and Koltunow, 2009), but asexual reproduction via seed (apomixis) (Asker and Jerling, 1992) nevertheless is widespread in 293 genera (Hojsgaard et al., 2014b). 
Sexual reproduction in angiosperm is initiated by double fertilization involving the fusion of reducing male and female gametes in the ovule and producing embryo and endosperm as the organ of seeds. In contrast, apomixis evolved seed formation by the development of functional female gamete without meiosis (apomeiosis), developing embryo without fertilization (parthenogenesis), and a functional endosperm. In terms of timing of development, apomixis is divided into gametophytic apomixis and sporophytic apomixis. The gametophytic apomixis is initiated early in gametophyte development during ovule development, but sporophytic apomixis develops at the later development of ovules or usually occurs in mature ovules (Koltunow, 1993). In gametophytic apomixis, diplospory is developed via restitution of meiosis, whereas apospory is developed from a non-meiotic cell of the nucellus. Both diplospory and apospory result in an embryo sac with an unreduced embryo and several unreduced polar nuclei (Nogler, 1984a; Asker and Jerling, 1992; Carman, 1997; Hojsgaard and Hörandl, 2019). In sporophytic apomixis, adventitious embryony is initiated directly from somatic cells in ovules tissues that do not belong to megagametophyte structure (Nogler, 1984b; Asker and Jerling, 1992; Koltunow, 1993). The seed formation of apomictic plants requires fertilization of polar nuclei (pseudogamy) or without fertilization (autonomously), and pseudogamy is usually combine with facultative apomixis (Hojsgaard and Hörandl, 2019).

\subsubsection{Stress effect on mode of reproduction}

Apomixis is expressed facultatively in most plants. The coexistence of sexuality and apomixis makes some degree of sexuality in apomixis plants possible. The formation of apomictic seeds alone as obligate apomixis is very rare (Asker and Jerling, 1992). In facultative apomixis, the level of viable asexual seed formation can vary considerably between individuals (Bicknell and Koltunow, 2004). Environmental factors play an important role in controlling reproduction and fitness trade-offs (Quarin, 1999; Šarhanová et al., 2012; Schinkel et al., 2016). Since plants are sessile organisms, they are exposse to unfavorable abiotic conditions such as severe drought, temperature, alkaline, light, nutrients (Shah et al., 2016). Increasing of sexuality of facultative apomixis after stress had been reported, e.g. in Eragrostis (Selva et al., 2020), Paspalum (Quarin, 1986; Delgado et al., 2016; Karunarathne et al., 2020), Boechera (Aliyu et al., 2010; Mateo de Arias, 2015; Gao, 2018; Carman et al., 2019), and in Ranunculus (Klatt et al., 2016; Klatt et al., 2018). Nevertheless, the mechanisms of how the stress alters the proportion of sexual and asexual seed formation are still not so well understood.

Environmental stress alters the accumulation of ROS, which triggers oxidative stress, and enhances the frequency of homologous recombination during meiosis (De Storme and Geelen, 2014). In germline precursor cells of diploid plants, mild oxidative stress may increase the level of DNA double-strand breaks (DSBs) as an initiator of meiosis (Hörandl and Hadacek, 
2013), while strong stress causes oxidative damage and abortion of gametophyte (De Storme and Geelen, 2014). In polyploids, however, a better stress tolerance might reduce the stimulus of meiosis, hence the asexual pathway to the apomictic seed formation as a surrogate of the sexual reproduction (Hörandl and Hadacek, 2013).

\subsection{The Ranunculus auricomus complex}

The Ranunculus auricomus complex is a Eurasian predominantly apomixis group that comprises ca. 800 polyploid apomictic species and four diploid sexual species (Hörandl, 1998). In central Europe, These species grow at a broad range of habitats from riverside area to forest margin and semi-dry anthropogenic meadow (Paun et al., 2006; Hörandl et al., 2009; Hodač et al., 2014; Hojsgaard et al., 2014a). R. auricomus is well-established a model system for studying reproduction modes and the mechanism that trigger evolution of sex and apomixis compared due to the higher frequency of facultative apomixis (Nogler, 1984b; Hörandl and Temsch, 2009; Aliyu et al., 2010; Hojsgaard et al., 2014a; Klatt et al., 2016). Our model system of $R$. auricomus complex comprises three cytotypes (diploid, tetraploid, and hexaploid) that closely related and genetically similar to sexual progenitor species and autopolyploid hybrid that originated from three species $(R$. cassubicifolius W. Koch, $R$. carpaticola Soó and $R$. notabilis notabilis Hörandl \& Guterm) (Paun et al., 2006; Hodač et al., 2014; Barke et al., 2018; Barke et al., 2020). Recently, Karbstein et al. (2020) proposed a taxonomic revision uniting the two former taxa under $R$. cassubicifolius, but for simplicity, I keep the original names.

The natural diploid apomictic in $R$. auricomus was not known; therefore, I use diploid plants of F2 synthetic hybrids crosses of the sexual taxa $R$. carpaticola $R$. notabilis (Barke et al., 2018; Barke et al., 2020) in this study. The tetraploid of $R$. variabilis plants are putative natural allopolyploids of the $R$. carpaticola/cassubicifolius lineage and $R$. notabilis lineage that occurs sympatrically with the parental species (Hodač et al., 2014). Tetraploids were grown from seeds of plants that were originally collected near Schönau, Mühlkreis, Austria (48²2'46.00"N $14^{\circ} 44^{\prime} 46.00 " E$, wet meadow) by L. Hodač and K. Spitzer (LH002, GOET). The hexaploids of $R$. carpaticola $\times$ cassubicifolius were grown from seeds of natural hybrids of $R$. carpaticola $\mathrm{x}$ R. cassubicifolius (original clone 29 from a forest margin and clone 35 from a meadow (Hörandl et al., 2009; Klatt et al., 2016). The hexaploids were previously studied for light stress effect on reproductive mode and metabolite alternation in the climatic chamber (Klatt et al., 2016). In the study, hexaploids were grown under a controlled environment that has been optimized following the original habitat of the provenance of $R$. auricomus complex in forest margin or understory habitats in Slovakia (Paun et al., 2006). I assume that three cytotypes of $R$. auricomus complex are pre-adapted to the same natural light condition since their 
progenitors and the natural hybrids cover the similar geographical area and altitudinal zone (Hörandl et al., 2009).

\subsection{Aims of the thesis}

This thesis aims to shed light on the effect of photoperiod stress on the mode of reproduction and photosynthesis performance and whether polyploidy alters stress response. Based on the model system of the facultative apomictic $R$. auricomus complex, I applied a comprehensive analysis to test how stress regulation differs between three cytotypes on the reproductive development within three-component gametophytic apomixis: 1) apomeiosis (formation of unreduced embryo sacs); 2) parthenogenesis (embryo development without fertilization of egg cell); 3) functional endosperm development with male genome contribution from the pollen (pseudogamously) or independent from pollen (autonomously), the treatments effect on pollen quality as the pollen-dependent character of the seed formation, and photoprotective mechanisms of the photosynthetic organ that support the reproductive tissue.

Chapter 2- The main objectives of this chapter were to assess the stress expression between the treatments on (1) the alternation of apomeiosis to meiosis; (2) the pollen quality: (3) the mode of seed formation; and (4) seed set and to evaluate the sign of buffer stress in polyploids. In this chapter, I also presented the plant's clonality and the relation among cytotypes based on the genotyping with simple sequence repeater (SSR).

Chapter 3- The main objective of chapter 3 was to examine the photoprotective mechanism of three cytotypes based on their alternation in chlorophyll a fluorescence measurement and to combine the result of photoperiod stress on photosynthesis performance with the alternation of ovule formation. 


\section{Chapter 2: Ploidy-dependent effects of light stress on the mode of reproduction in the Ranunculus auricomus complex (Ranunculaceae)}

Fuad Bahrul Ulum, Camila Castro, Elvira Hörandl

Published in Frontiers in Plant Science 11 (2020): 104

DOI:10.3389/fpls.2020.00104

\subsection{Abstract}

Polyploidy in angiosperms is an influential factor to trigger apomixis, the reproduction of asexual seeds. Apomixis is usually facultative, which means that both sexual and apomictic seeds can be formed by the same plant. Environmental abiotic stress, e.g. light stress, can change the frequency of apomixis. Previous work suggested effects of stress treatments on meiosis and megasporogenesis. We hypothesized that polyploidy would alter the stress response and hence reproductive phenotypes of different cytotypes. The main aims of this research were to explore with prolonged photoperiods, whether polyploidy alters proportions of sexual ovule and sexual seed formation under light stress conditions. We used three facultative apomictic, pseudogamous cytotypes of the Ranunculus auricomus complex (diploid, tetraploid, and hexaploid). Stress treatments were applied by extended light periods (16.5 h) and control $(10 \mathrm{~h})$ in climate growth chambers. Proportions of apomeiotic vs. meiotic development in the ovule were evaluated with clearing methods, and mode of seed formation was examined by single seed flow cytometric seed screening (sSFCSS). We further studied pollen stainability to understand effects of pollen quality on seed formation. Results revealed that under extended photoperiod, all cytotypes produced significantly more sexual ovules than in the control, with strongest effects on diploids. The stress treatment affected neither the frequency of seed set nor the proportion of sexual seeds nor pollen quality. Successful seed formation appears to be dependent on balanced maternal: paternal genome contributions. Diploid cytotypes had mostly sexual seed formation, while polyploid cytotypes formed predominantly apomictic seeds. Pollen quality was in hexaploids better than in diploid and tetraploids. These findings confirm our hypothesis that megasporogenesis is triggered by light stress treatments. Comparisons of cytotypes support the hypothesis that ovule development in polyploid plants is less sensitive to prolonged photoperiods and responds to a lesser extent with sexual ovule formation. Polyploids may better buffer environmental stress, which releases the potential for aposporous ovule development from somatic cells, and may facilitate the establishment of apomictic seed formation.

Keywords: apomixis, ssFCSS, light stress, meiosis, pollen, polyploidy, Ranunculus, seed formation 


\subsection{Introduction}

Polyploidy is a heritable trait of obtaining more than two sets of chromosomes in the nuclei (Comai, 2005). A polyploid arises either from intraspecific genome duplication (autopolyploidy) or the merging of the genome of distinct species through hybridization and subsequent genome duplication (allopolyploidy) (Grant, 1981). Polyploidy is quite common in flowering plants, estimated to occur in more than $50 \%$ of species (Soltis et al., 2015) and is considered as a major factor in plant evolution (Soltis et al., 2014). Even though polyploidy is potentially obstructed by several disadvantages, e.g., disruption effects of structural enlargement of nuclei, side effects of aneuploidy, and epigenetic mutation, it also provides advantages such as heterosis, gene redundancy, and novel gene combinations. Heterosis favors polyploids that are more vigorous than their diploid progenitors, while gene redundancy protects polyploids from the deleterious effect of mutation (Comai, 2005).

Polyploidisation, with higher DNA content, increases the cell size and promotes diversity of the genome, transcriptome, and metabolome. These improvements imply a greater resistance to environmental change (Schoenfelder and Fox, 2015). Several studies reported a better adaptivity of polyploid plants to abiotic stress conditions, such as salt (Chao et al., 2013), drought (del Pozo and Ramirez-Parra, 2014; Martínez et al., 2018), drought and heat stress (Godfree et al., 2017), cold (Klatt et al., 2018), and light (Coate et al., 2012). The better stress response and adaptation of polyploids to abiotic conditions are probably under epigenetic control (del Pozo and Ramirez-Parra, 2014). Polyploidy changes the methylation profile under stressful environments, as reported, e.g. for Brassica napus after drought (Jiang et al., 2019).

Notably, stress conditions can also influence mode of reproduction, especially apomixis, the asexual reproduction via seed (Nogler, 1984a). Apomixis is widespread in angiosperms (Hojsgaard et al., 2014b) and occurs most frequently in polyploid cytotypes, but occasionally also in diploids (Grant, 1981; Carman, 1997; Hojsgaard and Hörandl, 2019). Gametophytic apomixis, the form of interest here, involves formation of an unreduced embryo sac from an unreduced megaspore via meiotic restitution of the megaspore mother cell (diplospory) or from a somatic cell of the nucellus tissue (apospory) (Asker and Jerling, 1992; Koltunow and Grossniklaus, 2003). Functional seed development through gametophytic apomixis involves three components: (1) apomeiosis (formation of unreduced embryo sac); (2) parthenogenesis (embryo development without fertilization of egg cell); and (3) functional endosperm development with male genome contributions from the pollen (pseudogamously) or independent from pollen (autonomously) (Nogler, 1984a). Male development is usually meiotic, but microsporogenesis is often disturbed, and hence final pollen quality is often strongly reduced (Asker and Jerling, 1992; Izmaiłow, 1996; Horandl et al., 1997; Mráz et al., 
2009). Apomixis is heritable (Ozias-Akins and van Dijk, 2007), and under genetic and epigenetic control (Grimanelli, 2012; Hand and Koltunow, 2014). Natural apomixis is frequently facultative, which means that the plant produces sexual and asexual seeds within one generation, often within the same flower or inflorescence (Bicknell et al., 2003; Aliyu et al., 2010; Cosendai and Hörandl, 2010; Hojsgaard et al., 2013; Schinkel et al., 2016).

Alternation of frequencies of asexual vs. sexual reproduction was observed under abiotic stress conditions, e.g., temperature, drought stress, salt stress, and photoperiod in many different genera (Evans and Knox, 1969; Saran and de Wet, 1976; Quarin, 1986; Gounaris et al., 1991; Klatt et al., 2016; Rodrigo et al., 2017; Klatt et al., 2018). Such a condition-dependent sex is also known from other asexual eukaryotes (Ram and Hadany, 2016). Abiotic stress leads to the accumulation of ROS (Reactive oxygen species) in plant tissues, which triggers oxidative damage, but also can initiate various epigenetic, genetic and hormonal signaling pathways for plant development (Halliwell, 2006; Foyer and Noctor, 2009; Huang et al., 2019). In the germline precursor cells, oxidative stress may increase the level of DNA double-strand breaks (DSBs) as initiator of meiosis. Here meiosis could act as DNA repair system (Hörandl and Hadacek, 2013). The above-mentioned studies on condition-dependent sex in plants support this hypothesis. In polyploids, however, an improved tolerance of stress conditions might decrease the stimulus for meiosis, and consequently trigger the alternative asexual development (Hörandl and Hadacek, 2013). However, a putative differential response of cytotypes to stress conditions with respect to mode of reproduction was so far not investigated.

We use here as a model system three cytotypes of the Ranunculus auricomus complex, a Eurasian polyploid complex with facultative, aposporous and pseudogamous apomixis (Nogler, 1984b; Hojsgaard et al., 2014a). In Central Europe, the R. auricomus complex comprises three closely related and genetically similar sexual progenitor species, and polyploid apomictic hybrids of these taxa (Hörandl et al., 2009; Hodač et al., 2014). One of the hexaploid hybrids ( $R$. carpaticola x cassubicifolius) with facultative apomixis (Hojsgaard et al., 2014a) was used previously for testing the response to light stress. This previous experiment using extended photoperiod enhanced sexual megaspore formation in these hexaploid $R$. auricomus clones concomitant with oxidative stress (Klatt et al., 2016). In our study, we test the hypothesis that with the light stress treatment, diploids would respond more intensively to stress conditions with higher frequencies of sexual development than higher ploidy levels. Here we extend the treatment of (Klatt et al., 2016) to diploid, lower polyploid (tetraploid), and the same hexaploid plants to observe effects on mode of reproduction in different ploidy levels. To simulate the effect of extended photoperiod on the components of gametophytic apomixis, we study here two developmental steps, namely ovule formation, and seed formation. Since 
microsporogenesis is meiotic without an alternative asexual developmental pathway, we focus here on pollen quality as a possible factor for successful seed formation. The main aims of this research are to explore with light stress treatments whether ploidy level alters stress response with respect to mode of reproduction, and whether stress response correlates positively to sexual megaspore formation and/or proportions of sexual seed formation.

\subsection{Materials and Methods}

\subsubsection{Plant material}

We used for the extended photoperiod experiment facultative apomictic plants of the Ranunculus auricomus complex from three different cytotypes. These cytotypes are hybrids that originated from three Central European parental species ( $R$. cassubicifolius, $R$. carpaticola, and $R$. notabilis). The diploid plants were synthetic F2 hybrids of $R$. carpaticola $x$ notabilis and represent sister or sibling individuals from two parental lines; see details of crossing design in Barke et al. (2018). We used these plants because natural diploid apomicts are not known for the $R$. auricomus complex. The tetraploids were garden offspring of Ranunculus variabilis, which is a putative natural allopolyploid of the $R$. carpaticola/cassubicifolius lineage and the $R$. notabilis lineage, and occurs sympatrically with the parental species in Central Europe (Hodač et al., 2014). The hexaploids were garden offspring of Ranunculus carpaticola $x$ cassubicifolius, the same plants as used by Klatt et al. (2016). Hence, all cytotypes are hybrids, and they share the genetic background of closely related parental species (Hörandl et al., 2009). Since the parental taxa and the natural hybrids occur all in the same geographical area and altitudinal zone (Hörandl et al., 2009), we can also assume that they are all pre-adapted to the same natural light conditions. The ploidy level of tetraploids was ascertained using flow cytometry following methods of (Klatt et al., 2016). A list of materials with an identity number and ploidy levels is given in the Appendix (Suppl. Table. 1). Plants were cultivated in the old botanical garden of the University of Goettingen from summer to winter for exposure to natural conditions, to stimulate the flower initiation.

\subsubsection{Growth Chamber Setup}

The plants were moved into the climate growth chamber when sprouting at the beginning of the spring season. We run experiments for two years to get a more complete sampling. The first-year experiment was started from the first week of March 2017; the second year was started from first February 2018. A total of c. 25 plants from each cytotype were grown with 10 -hour photoperiod (control) and 16 plus 0.5 hours photoperiod (stress treatment) following (Klatt et al., 2016). Temperature setup and relative humidity were kept stable at $18^{\circ} \mathrm{C}$ and 60 $\%$ respectively. The light intensity was measured with a photometer (3415F Quantum Light 
Meter, Spectrum Technologies, Inc, Plainfield, USA) as photoactive radiation (PAR) c. 250 $\mu \mathrm{mol} \mathrm{m} \mathrm{m}^{-2} \mathrm{~s}^{-1}$ (measured at shoot tips).

\subsubsection{Plant Genotyping}

Genotyping by Simple sequence repeats (SSRs) was applied to verify the plant's clonality and the relationships of cytotypes. We conducted SSRs only to tetraploid plants following methods by (Klatt et al., 2016). The SSR data for the other two cytotypes were derived from (Barke et al., 2018) for diploids and (Klatt et al., 2016) for hexaploids. Genomic DNA was performed by extracting dried leaf samples using Invisorb ${ }^{\circledR}$ Spin Plat Mini Kit (Qiagen, Hilden, Germany) according to the manufacturer's protocol. Multiplex Polymerase Chain reaction (PCR) was conducted at $25 \mu$ volumes, containing $1 \mu \mathrm{l}$ template DNA, 12.5 Roti $^{{ }}$-Pol TaqS Master mix (Carl Roth $\mathrm{GmbH}+$ Co. KG, Karlsruhe, Germany), $1 \mu$ I Forward Primer, $1 \mu$ l Reverse Primer, $0.125 \mu \mathrm{MgCl}_{2}, 1 \mu \mathrm{l} \mathrm{CAG-Primer} \mathrm{(FAM} \mathrm{or} \mathrm{HEX} \mathrm{labeled).} \mathrm{PCR} \mathrm{reactions} \mathrm{were} \mathrm{run} \mathrm{in} \mathrm{a}$ BIORAT TM Thermal Cycler. PRC machine setting was: $94{ }^{\circ} \mathrm{C}$ for $10 \mathrm{~min}$, then $14 \mathrm{x}$ (denaturation at $94^{\circ} \mathrm{C}$ for $60 \mathrm{~s}$, annealing at $62^{\circ} \mathrm{C}+0.5^{\circ} \mathrm{C}$ per cycle for $90 \mathrm{~s}$, extension at 72 ${ }^{\circ} \mathrm{C}$ for $60 \mathrm{~s}$ ), followed subsequently by $35 \times$ (denaturation at $94^{\circ} \mathrm{C}$ for $30 \mathrm{~s}$, annealing at $55^{\circ} \mathrm{C}$ for $30 \mathrm{~s}$ and extension at $72{ }^{\circ} \mathrm{C}$ for $30 \mathrm{~s}$ ), last extension step at $72{ }^{\circ} \mathrm{C}$ for $60 \mathrm{~s}$ and final storage conditions at $4{ }^{\circ} \mathrm{C}$. PCR samples were adjusted before $85 \mu$ formamide (HiDi) was added. This mixture was run in an automatic capillarity sequencer Genetic Analyzer 3130 (Applied Biosystems, Forster City, CA, USA) using Gene Scan 500 Rox (Applied Biosystems) as size standard after a denaturing pretreatment for $3 \mathrm{~min}$ at $92^{\circ} \mathrm{C}$. Scoring of the electropherograms was done using Genemarker V2.4.2 (SoftGenetics LLC, State College, PA, USA) and exported as a binary matrix presence/absence of alleles to characterize multilocus genotypes. We applied Neighbour-joining analysis based on Jaccard similarity index in FAMD to test the SSR profiles (Schlüter and Harris, 2006). Branch support values were derived from the majority consensus tree from 1000 bootstrap replicates. FigTree v1.4.2 (Rambaut, 2007) visualized the result.

\subsubsection{Female development}

Development of embryo sacs was already previously characterized within the R. auricomus complex on both apomictic and sexual species and is quite uniform (Nogler, 1984a; Hojsgaard et al., 2014a; Klatt et al., 2016; Barke et al., 2018): the megaspore mother cell differentiates near the micropyle and undergoes meiosis, resulting in a megaspore tetrad. In sexual development, only the chalazal megaspore develops further and produces after three mitotic divisions a typical 7-celled, 8-nucleate Polygonum type embryo sac (with three antipodals, a binucleate central cell, two synergids, and one egg cell). Apomictic development is characterized by enlargement of a somatic cell in the nucellus which emerges in parallel and 
aside the megaspore tetrad, and continues embryo sac development into an unreduced Polygonum type embryo sac, whereas all megaspores abort. Embryological analysis of the female development was made at the end of sporogenesis and the beginning of gametogenesis, following Hojsgaard et al., (2014a) and Barke et al. (2018). R. variabilis, the only taxon that was analyzed for the first time here, did not show any deviations in timing or type of development. Flower buds were fixed at Formalin : acetic acid : ethanol : $\mathrm{dH}_{2} \mathrm{O}(2: 1$ : 10 : 3.5) (FAA) for $48 \mathrm{~h}$, and stored in $75 \%$ ethanol (Hojsgaard et al., 2014a). The flower bud was treated by dehydrating in four steps of $30 \mathrm{~min}$ incubation in $1 \mathrm{ml}$ of $70 \%, 95 \%$, and $100 \%$ (two times). Then the flower buds were treated by clearing method in five steps of $30 \mathrm{~min}$ in $300 \mu \mathrm{l}$ of upgrading series of methyl salicylate diluted in ethanol $(25 \%, 50 \%, 70 \%, 85 \%$, and $100 \%$ ) (Young et al., 1979). The perianth of selected flower buds was removed, ovaries were dissected and mounted in methyl salicylate on glass slides. Female sporogenesis and early stages of sexual or aposporous gametophyte development were analysed with differential interface contrast (DIC) in a light transmission microscope (Leica DM5500B with DFC 450 Camera, LAS V41 software, Leica Microsystems, Wetzlar, Germany). The determination of sexual and asexual ovules was made by the absence or presence of aposporous initial cells (AIC), respectively (van Baarlen et al., 2002). We excluded ovules with unclear structure and aborted ones. We only considered the data from a plant that had a minimum of five observable ovules. Additional data from (Klatt et al., 2016) were added to increase the $\mathrm{N}$ value for the hexaploid cytotype.

\subsubsection{Seed set}

After we collected the sample for embryological analysis, the remaining flowers were then manually pollinated to increase fertilization rates. In fruiting stages, we bagged a minimum of five peduncles with collective fruits with porous plastic bags to avoid seed loss. We harvested the mature collective fruits and evaluated the proportion of well-developed seeds (seed-set percentage) among ploidies per flower on individual according to Hörandl (2008). Welldeveloped seeds were stored at room temperature and were used for reproductive pathway analysis.

\subsubsection{Reproductive pathway of seed formation}

The reproductive pathway was evaluated by single seed flow cytometric seed screening (ssFCSS) (Matzk et al., 2000). Two steel balls grounded a single seed ( $\varnothing 4 \mathrm{~mm}$ ) in a $2 \mathrm{ml}$ Eppendorf tube in a TissueLyzer II (Qiagen, Hilden, Germany; $30 \mathrm{~Hz} \mathrm{s-1}$ for $7 \mathrm{~s}$ ). Nuclear isolation and staining were attained in two steps using Otto buffers (Otto, 1990). In the first step, nuclear isolation, $200 \mu$ l Otto I buffer $(0.1 \mathrm{M}$ citric acid monohydrate, $0.5 \% \mathrm{v} / \mathrm{v}$ Tween 20) was added and hand shacked with the ground material for $30 \mathrm{~s}$. The solution was then 
filtered (30 $\mu \mathrm{m}$ mesh, Celltrics ${ }^{\circledR}$ Münster, Germany) into plastic tubes (3.5 ml, $55 \mathrm{~mm}$ x $12 \mathrm{~mm}$, Sarstedt, Nümbrecht, Germany). In the second step, staining, $800 \mu \mathrm{l}$ otto II buffer (0.4 M $\mathrm{Na}_{2} \mathrm{HPO}_{2}, \mathrm{ddH}_{2} \mathrm{O}$ and charged with $3 \mathrm{ng} / \mathrm{ml}$ 4,6-diamidinophenyl-indole (Sigma-Aldrich, Munich, Germany)) was added to the filtrate, and the solution was measured directly in Flow cytometer (CyFlow ${ }^{\circledR}$ Ploidy Analyser (Sysmex Partec GmbH, Görlitz, Germany) in the Blue fluorescence (UV LED, gain 365). Histograms were analyzed using CyView ${ }^{\mathrm{TM}}$ V.1.6 software (Partec $\mathrm{GmbH}$ ). The coefficients of variation were less than $8 \%$. The ploidy levels of embryo and endosperm were determined, and peak indices (PI) (mean peak value of the embryo compared to the mean peak of endosperm) were assessed (Suppl. Fig. 5). For a Polygonum type embryo sac with two polar nuclei, the peak index for a sexual seed is c. 1.5, while for asexual seeds it can be $2.0,2.5$, or 3.0 , depending on the contribution of pollen nuclei to endosperm formation. We observed the following developmental pathways: Sexual, pseudogamous apomixis, autonomous apomixis, and $\mathrm{B}_{\mathrm{II}}$-hybrids (Hojsgaard and Hörandl, 2019). $B_{\| I}$-hybrids arise from an unreduced embryo sac, whereby egg cell and polar nuclei were fertilized. The $B_{111}$-hybrids were excluded for the determination of the proportion of sexual seeds since this mode of reproduction is intermediate between sexual and asexual seed formation.

\subsubsection{Pollen stainability}

Pollen stainability was determined on a minimum of 500 pollen grains per plant from all cytotypes in both chambers by using $10 \%$ Lugol's iodine $\left(\mathrm{I}_{2} \mathrm{KI}\right)$ solution, following methods by (Schinkel et al., 2017). The stainability of starch content was used as an indicator of viable pollen under a light microscope (LEICA DM5500B with DFC $450 \mathrm{C}$ camera, LAS V41 software, Leica Microsystems, Wetzlar, Germany) at 400x magnification. The viable pollen grains were indicated by black-stained color, but brownish, reddish, and translucent (empty) pollen was counted as non-viable.

\subsubsection{Statistical analyses}

All data were tested for their normality distribution by Kolmogorov-Smirnov and Shapiro-Wisk test and for their homogeneity of variance with the Levene test. Female development, seed set, reproduction pathway of seed formation, and pollen viability were determined per flower as a percentage and subsequently averaged per plants. The percentage of data were arcsine transformed before statistical analysis. We tested the influence of treatment on mean sexual ovules and seed set among ploidies with General Linear Model (GLM) univariate (Two-way ANOVA) for completely randomized factorial design model, and means were compared according to the least significant difference (LSD) test at 0.05 probability level ( $p$-value $<0.05$ ). Tukey HSD was performed to the means of sexual ovules to determine the main factors. 
Nonparametric Kruskal-Wallis and Mann-Whitney U-test were applied to test the influence of treatment on sexual seed formation per ploidy. Boxplots were plotted with untransformed percentage values and show the $25^{\text {th }}$, and $75^{\text {th }}$ percentile ranges as a box, and the median as a black line: circles are outliers; asterisks are extreme values. All statistical analyses were performed with IBM SPSS Statistic 25 (IBM Deutschland GmbH).

\subsection{Results}

\subsubsection{Female Development}

The ovule development of all three cytotypes of the $R$. auricomus complex showed the same pattern of a typical Polygonum type embryo sac (Suppl. Figs. 1-4). We had observed 6,505 ovules (c. 18 ovules per flower bud) among cytotypes at megasporogenesis and early megagametogenesis. At this stage, sexual and asexual ovules can be discriminated (Suppl. Fig. 4). At the megasporogenesis stage, a meiotic division of a megaspore mother cell produced four cells, i.e. a megaspore tetrad. During the next step, three cells aborted, and only the chalazal cell remained as functional megaspore. At megagametogenesis stage, the functional megaspore enlarged with the presence of vacuoles and continued with three nuclear divisions, resulting in a total of eight nuclei. Development of sexual ovules was indicated by the absence of any aposporous initial cell (AIC) during megasporogenesis and early megagametogenesis. On the other hand, in asexual ovules, one or more AIC was observed directly near the megaspores at the chalazal pole or near to this area, but at a different optical layer (Figure 2.1).

\subsubsection{Effects of ploidy, treatment, and combined effect of ploidy/treatment to the proportion of female development}

Extended photoperiod enhanced the proportion of sexual ovules in all three cytotypes of the $R$. auricomus complex. The mean proportion of sexual ovules significantly increased from control treatment to stress treatment (80.37 (mean) \pm 19.38 (sd) \% to $99.26 \pm 1.26 \%$; p-value $<0.001)$ in diploid, $(57.90 \pm 8.79 \%$ to $80.29 \pm 10.67 \%$; p-value < 0.001$)$ in tetraploids, and $52.61 \pm 26.11 \%$ to $70.36 \pm 20.04 \%$; p-value $=0.006$ ) in hexaploids (Figure 2.2). ANOVA revealed significant alterations by the main effect photoperiod ( $p$-value $<0.001$ ) and ploidy ( $p$ value $<0.001$ ), but not by the interrelationship between them ( $p$-value $=n s)$. Tukey HSD revealed significant differences in control treatment between diploids and hexaploids ( $p$-value $=0.047$ ) and in stress treatment between diploids and polyploids ( $p$-value $<0.001$ ) but there is neither a significant difference between tetraploids and hexaploids in the both treatments nor among diploids and tetraploid in the control treatment ( $p$-value =ns) (Suppl. Table 3). 

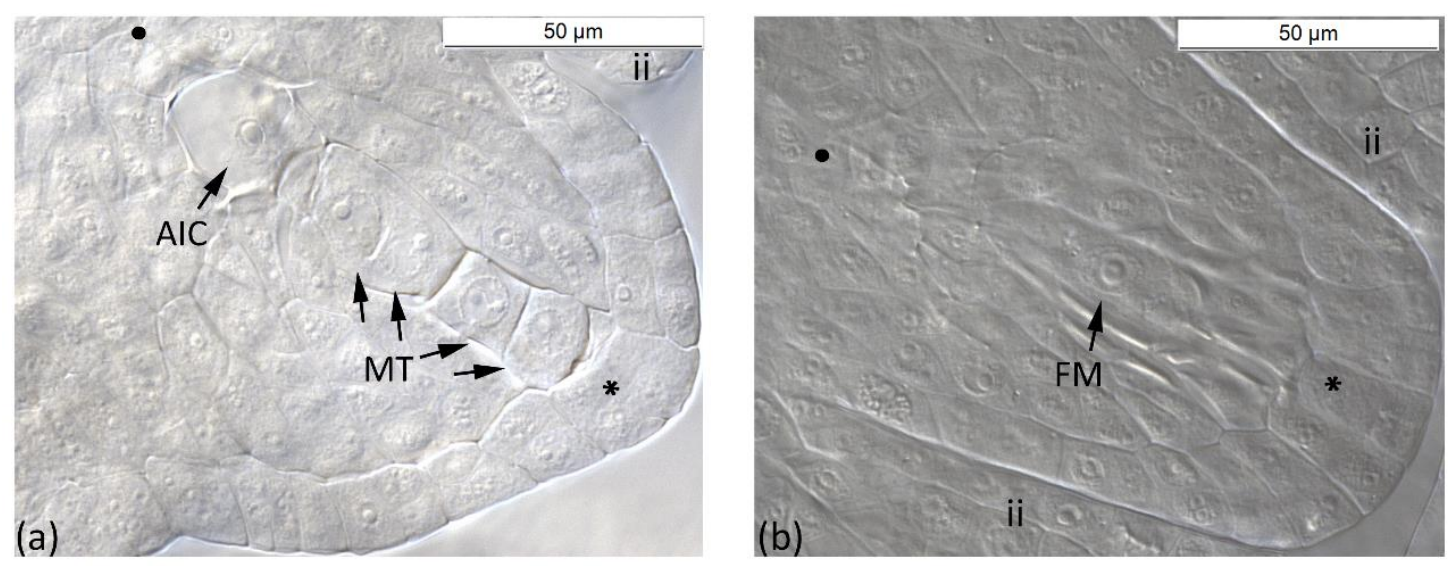

Figure 2.1 Megasporogenesis of $R$. variabilis plants. (a) Asexual ovule during megaspore formation. The germline with megasporocyte tetrad and one aposporous initial cell near the chalazal pole is shown. (b) Sexual ovule during functional megaspore formation. Only one cell near the chalazal pole survived and developed into a functional megaspore whereas the other three cells are aborted. Plant individual: (a) LH1406030B4-7 (Tetraploid); (b) LH1406030B419 (Tetraploid). AIC, Aposporous Initial Cell; FM, Functional Megaspore; ii, inner integument; MT, Megaspore Tetrad; SY, Synergid; •, chalazal pole; " , micropylar pole. Scale bar: $50 \mu \mathrm{m}$.

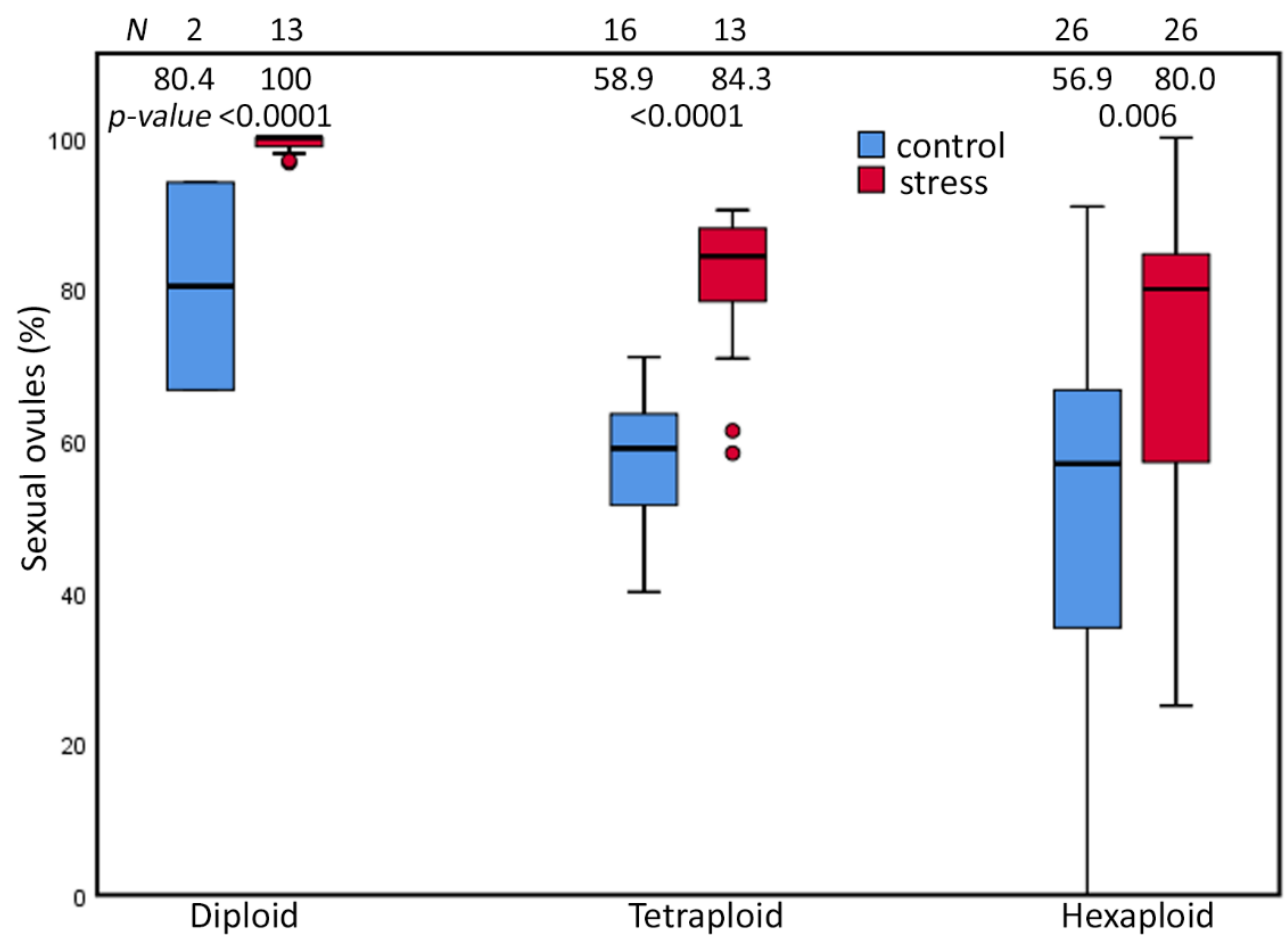

Figure 2.2 Proportions of sexual ovules in the $R$. auricomus complex plants grown in climatic chamber under prolonged photoperiod (stress) and shortened photoperiod (control). Mean values and statistical significance are given in figure. $\mathrm{N}=$ number of individuals. For the test statistics, see Suppl. Table 2. 
Table 2.1 P-values for the two way ANOVAs to determine the interaction effect of stress treatment and ploidy level on the proportion of sexual ovules.

\begin{tabular}{llllll}
\hline Source & Type III Sum of Squares & df & Mean Square & F & Sig. \\
\hline Ploidy & 1.769 & 2 & 0.885 & 14.091 & 0.001 \\
Treatment & 1.529 & 1 & 1.529 & 24.357 & 0.001 \\
Ploidy x Treatment & 0.132 & 2 & 0.066 & 1.053 & 0.353 \\
\hline
\end{tabular}

a. $\mathrm{R}$ Squared $=0.574$ (Adjusted R Squared $=0.551$ )

\subsubsection{Seed set}

Extended photoperiod did not influence the proportion of well-developed seeds among cytotypes of $R$. auricomus complex. Our investigation of 83 individuals revealed that no significant difference in seed set between plants grown in control and stress chamber ( $p$-value = ns) (Figure 2.3). Diploid plants under stress treatment produced a higher mean but not significant different proportions of well-developed seeds (mean value $=50.22 \%$ ) compared to control treatments (mean value $=39.84 \% ; p$-value $=0.300$ ). Tetraploid plants under stress treatment produced a mean of $28.97 \%$ compared to a mean of $31.09 \%$ ( $p$-value $=0.459$ ) under control treatment. Hexaploid plants under stress treatment produced a mean of 43.04 $\%$ compared to a mean of $42.17 \%(p$-value $=0.880)$ under control treatment. A two-way ANOVA revealed only significant differences between the ploidies ( $p$-value $<0.001$ ), but neither a significant effect on treatment nor an interaction effect ( $p$-value $=n s$ ) (Suppl. Table 4). Multiple comparison tests revealed that significant differences were observed between diploids and tetraploids ( $p$-value $<0.001$; Tukey HSD) and between tetraploids and hexaploids (p-value < 0.001; Tukey HSD) (Suppl. Table 5).

\subsubsection{Reproductive pathways of seed formation}

Extended photoperiod did not enhance the proportion of sexual seed over ploidies. The mean value of the proportion of sexual seeds was not significantly different between treatments among ploidies ( $p$-value $=n s$, Mann-Whitney U-test) (Figure 2.4, Suppl. Table 6). Analysis of 1,468 seeds among ploidies indicated several reproductive pathways in the $R$. auricomus complex (Table 2.2). In diploid plants, the majority of seeds was formed sexually while in tetraploid and hexaploid plants, asexuality was the most frequent reproduction mode (Figure 2.4). In diploid sexual seeds, we observed the ratio of embryo to endosperm DNA content of $2 \mathrm{C}: 3 \mathrm{C}$, which is the indication of double fertilization between reduced egg cell with one sperm cell $(1(m)+1(p))$ and two polar nuclei with the other sperm cell $(1(m)+1(m)+1(p))$, producing a Peak Index (PI) of 1.5. A few apomictic seeds were observed (two with pseudogamous endosperm and one with autonomous endosperm) only in the stress treatment. The pseudogamous endosperm comes from the development of an unreduced embryo $(2(\mathrm{~m}))$ and fertilization of two polar nuclei with one or two reduced or unreduced sperm cells 
$(2(m)+2(m)+1(p)$ or $2(p))$, with ratios of embryo to endosperm of $2 \mathrm{C}: 5 \mathrm{C}(\mathrm{PI}=2.5)$ and $2 \mathrm{C}: 6 \mathrm{C}$ $(P I=3.0)$. Autonomous endosperm develops from an unreduced embryo $(2(m))$ and unfertilized of two polar nuclei $(2 \mathrm{Cm}+2 \mathrm{Cm})$ with the ratio of embryo to endosperm of $2 \mathrm{C}: 4 \mathrm{C}$ $(P I=2.0)$, which is caused by the absence of paternal genome in seed development.

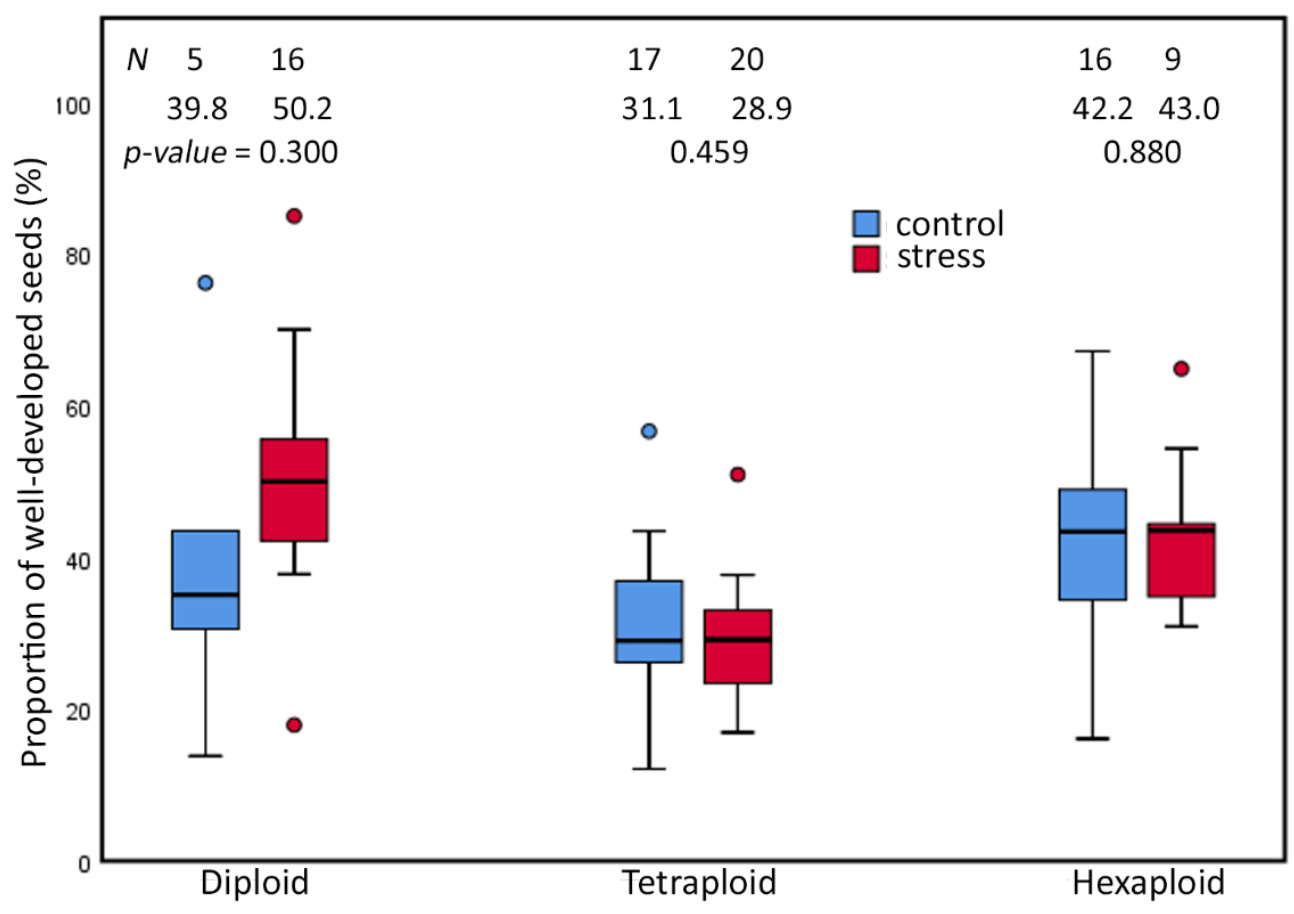

Figure 2.3 Proportions of well-developed seeds in the $R$. auricomus complex plants grown in climatic chambers under prolonged photoperiod (stress) and shortened photoperiod (control). Mean values and statistical significance are given in figure. $\mathrm{N}=$ number of individuals. For the test statistic, see Suppl. Table 2.

Tetraploid and hexaploid plants displayed more variation on the mode of seed reproduction. Sexual reproduction mode was present in 39 (6.2\%) tetraploid seeds and 36 (7.5\%) hexaploid seeds. Pseudogamous endosperm was the most frequent mode of seed formation and appeared in 543 (86.3\%) tetraploid seeds and 433 (90.7\%) hexaploid seeds. Generally, this mode of reproduction produced a PI value of 3.0. The less frequent forms of pseudogamous endosperm with a $\mathrm{PI}=2.5$ and $\mathrm{PI}=4.0$ originated from the contribution of one reduced sperm nucleus or two unreduced sperm nuclei. Autonomous endosperms $(P I=2.0)$ were the most infrequent mode of seed formation, in a total of four seeds $(0.55 \%)$ from tetraploids and nine seeds (1.93\%) from hexaploids. Another type of reproduction mode, i.e. partial apomixis with an unreduced egg cell fertilized by reduced pollen ( $B_{\| 1}$-hybrid), was more frequent in tetraploid plants (45 seeds or $12.43 \%$ ) compared with only one case in hexaploid plants (Table 2.2). 


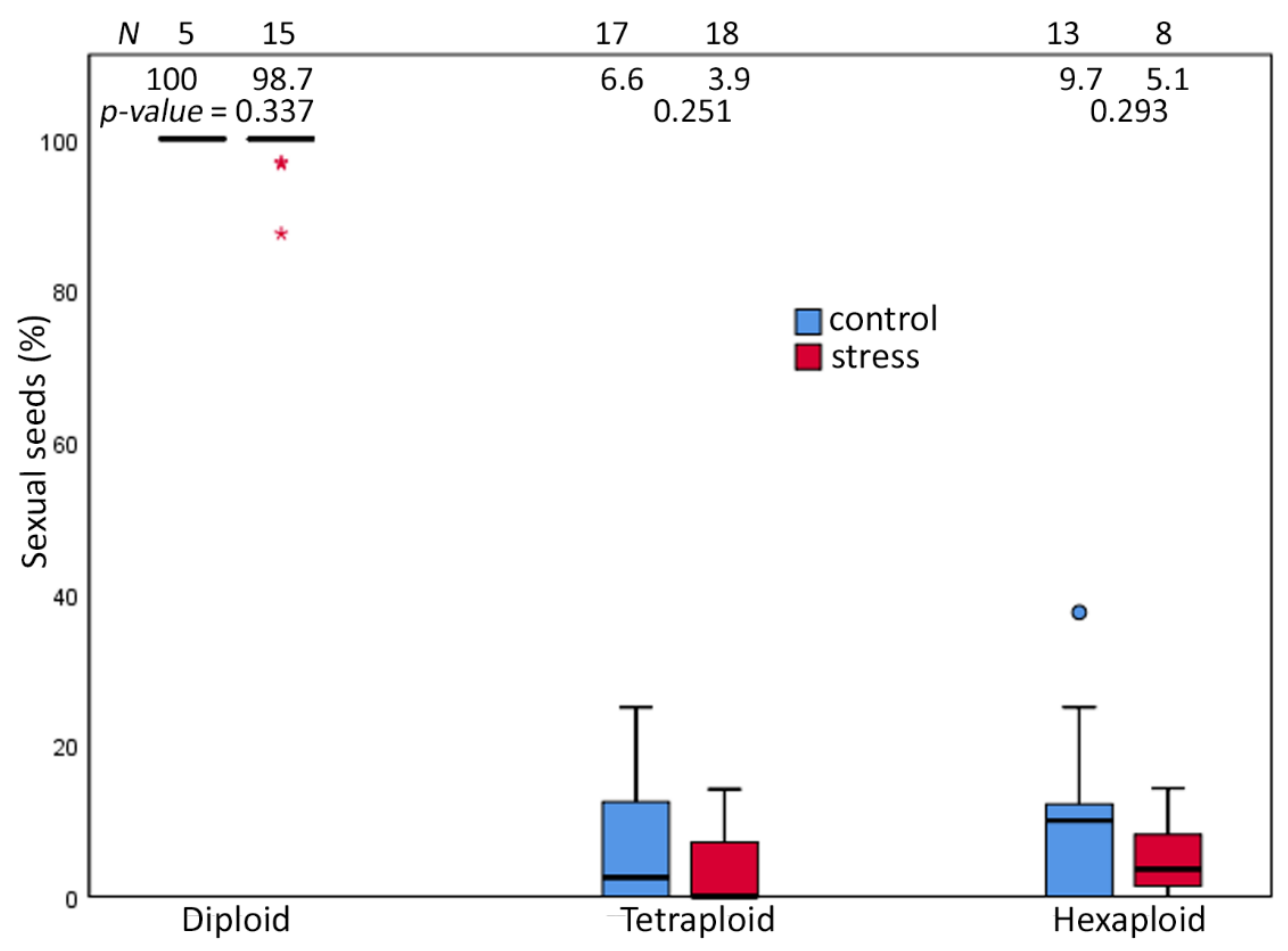

Figure 2.4 Proportions of sexual seeds in the $R$. auricomus complex plants grown in climatic chambers under prolonged photoperiod (stress) and shortened photoperiod (control). Mean values and statistical significance are given in figure. $\mathrm{N}=$ number of individuals. For the test statistic, see Suppl. Table 2. 
Table 2.2 Observed reproductive pathways of three cytotypes of the $R$. auricomus complex. Cx reflects ploidy based on DNA content : $m$. maternal genome contribution; p. paternal genome contribution. PI, peak index.

\begin{tabular}{|c|c|c|c|c|c|c|c|c|}
\hline \multirow{2}{*}{ Ploidy } & \multirow{2}{*}{$\begin{array}{l}\text { Reproduction } \\
\text { mode }\end{array}$} & \multicolumn{3}{|c|}{ Genome contribution to embryo/endosperm } & \multirow{2}{*}{$\begin{array}{l}\text { Sperm nuclei } \\
\text { contribution to } \\
\text { endosperm }\end{array}$} & \multirow{2}{*}{ PI } & \multicolumn{2}{|c|}{$\begin{array}{l}\text { Number of observations } \\
\text { (ssFCSS) }\end{array}$} \\
\hline & & Embryo (Cx) & Endosperm (Cx) & Em:End & & & Control & Stress \\
\hline \multirow[t]{4}{*}{ Diploid } & Sexual & $1(m)+1(p)$ & $2(m)+1(p)$ & 2C:3C & 1 & 1.5 & 77 & 282 \\
\hline & Apomictic & $2(m)$ & $2(m)+2(m) \dagger$ & $2 \mathrm{C}: 4 \mathrm{C}$ & 0 & 2 & 0 & 1 \\
\hline & & $2(m)$ & $4(m)+1(p) \ddagger$ & $2 C: 5 C$ & 1 & 2.5 & 0 & 1 \\
\hline & & $2(m)$ & $\begin{array}{l}4(m)+1(p)+ \\
1(p) \text { or } 4(m)+2(p) \ddagger\end{array}$ & $2 C: 6 C$ & 2 or 1 & 3 & 0 & 1 \\
\hline \multirow[t]{7}{*}{ Tetraploid } & Sexual & $2(m)+2(p)$ & $4(m)+2(p)$ & $4 \mathrm{C}: 6 \mathrm{C}$ & 1 & 1.5 & 19 & 20 \\
\hline & Apomictic & $4(m)$ & $4(m)+4(m) \dagger$ & $4 \mathrm{C}: 8 \mathrm{C}$ & 0 & 2 & 0 & 2 \\
\hline & & $4(m)$ & $8(m)+2(p) \ddagger$ & $4 C: 10 C$ & 1 & 2.5 & 10 & 24 \\
\hline & & $4(m)$ & $\begin{array}{l}8(m)+2(p)+ \\
2(p) \text { or } 8(m)+4(p) \ddagger\end{array}$ & $4 C: 12 C$ & 2 or 1 & 3 & 196 & 307 \\
\hline & & $4(m)$ & $8(m)+4(p)+4(p) \ddagger$ & $4 C: 16 C$ & 2 & 4 & 2 & 4 \\
\hline & BIII- hybrid & $4(m)+2(p)$ & $8(m)+2(p)+2(p)$ & $6 C: 12 C$ & 2 & 2 & 22 & 3 \\
\hline & & $4(m)+2(p)$ & $8(m)+2(p)$ & $6 C: 10 C$ & 1 & 1.6 & 5 & 15 \\
\hline \multirow[t]{6}{*}{ Hexaploid } & Sexual & $3(m)+3(p)$ & $6(m)+3(p)$ & $6 \mathrm{C}: 9 \mathrm{C}$ & 1 & 1.5 & 22 & 14 \\
\hline & Apomictic & $6(\mathrm{~m})$ & $6(m)+6(m) \dagger$ & $6 C: 12 C$ & 0 & 2 & 5 & 2 \\
\hline & & $6(m)$ & $12(m)+3(p) \ddagger$ & $6 C: 15 C$ & 1 & 2.5 & 20 & 19 \\
\hline & & $6(m)$ & $\begin{array}{l}12(m)+3(p)+3(p) \\
\text { or } 12(m)+6(p) \ddagger\end{array}$ & $6 C: 18 C$ & 2 or 1 & 3 & 246 & 142 \\
\hline & & $6(m)$ & $12(m)+6(p)+6(p) \ddagger$ & $6 C: 24 C$ & 2 & 4 & 3 & 3 \\
\hline & BIII- hybrid & $6(m)+3(p)$ & $\begin{array}{l}12(m)+3(p)+3(p) \\
\text { or } 12(m)+6(p)\end{array}$ & $9 \mathrm{C}: 18 \mathrm{C}$ & 2 or 1 & 2 & 1 & 0 \\
\hline
\end{tabular}

† Autonomous endosperm

† Pseudogamous endosperm, polar nuclei were fertilized by one reduced/unreduced or two reduced/unreduced sperm nuclei 


\subsubsection{Pollen stainability}

Extended photoperiod did not alter the proportion of viable pollen between treatments. The assessment through 34,348 pollen grains from 67 plants revealed no significant differences in pollen viability between plants of the same cytotype grown in both treatments ( $p$-value = ns; see Suppl. Fig. 6). Hexaploids produced a higher mean proportion of viable pollen (mean value $=64.6 \%$ in control treatment and $60.7 \%$ in stress treatment) compared to diploids (49.9\% in control treatment and $52.9 \%$ in stress treatment) and tetraploids (50.3\% in control treatment and $52.4 \%$ in stress treatment). Multiple comparison tests among ploidies revealed that the only significant differences were observed between tetraploid and hexaploid plants (p-value < 0.001; Tukey HSD; Suppl. Table 7).

\subsection{Discussion}

Mode of reproduction in the facultative apomictic plant is influenced by abiotic stress, e.g. by light (Knox, 1967; Saran and de Wet, 1976; Quarin, 1986; Klatt et al., 2016). However, these studies compared stress and control treatments only within the same cytotype. Under the same conditions, the degree of facultative apomixis is usually related to ploidy level (Delgado et al., 2016; Kaushal et al., 2018). In this study, we presented for the first time developmental patterns among three cytotypes of the $R$. auricomus complex under stress and control conditions. We tested the hypotheses that prolonged photoperiod enhances only the first component of apomixis, i.e., apomeiotic embryo sac development, with the expectation of a buffer effect of stress in polyploids. The other two apomixis components, i.e. parthenogenesis and endosperm development, were not affected by different photoperiods.

\subsubsection{Effects of ploidy, treatment, and combined effect of ploidy/treatment to the proportion of female development}

Prolonged photoperiod enhanced the proportion of sexual ovules, with a greater effect on diploids but lesser effect on tetraploids and hexaploids. Enhancement on the proportion of sexual ovules after the same type of light stress had been reported before only in the hexaploid cytotype (Klatt et al., 2016). The hexaploids also formed a comparable proportion of sexual ovules under garden conditions (Hojsgaard et al., 2014b). The three cytotypes of the $R$. auricomus complex exhibited a similar mode of reproduction as the pairwise comparison of data revealed insignificant differences between ploidies in control treatments. The result of controls and also the high genetic similarity of the three cytotypes (Suppl. Fig. 7) make it unlikely that slightly different genetic backgrounds of the cytotypes had influenced the results of our experiments. The proportion of sexual ovules of the diploid cytotype grown in the garden, ranging from $45 \%$ to $82 \%$ (Barke et al., 2018), was still within the range of our data. These plants represent recently formed synthetic F2 hybrids (Barke et al., 2018) with lower 
proportions of apospory than in the polyploids that already had established apomixis in the natural source populations. However, despite these more lineage-specific features, differential effects of treatments were observed in all three cytotypes in the early stages of development.

The prolonged photoperiod (16 plus $0.5 \mathrm{~h}$ ) may have expanded the accumulation of ROS (Reactive oxygen species) in the reproductive tissue, as reported for the hexaploids based on analysis of secondary metabolite profiles (Klatt et al., 2016). Results support the hypothesis that the oxidative lesions might mobilize the meiotic DNA repair system in the megaspore mother cell and trigger meiosis and megasporogenesis (Hörandl and Hadacek, 2013). This stimulus might increase the proportion of functional megaspores as a cellular survival strategy for the germline (Rodrigo et al., 2017), as shown remarkably in our diploids. Differential genetic stress regulation of sexual and apomictic plants was also observed in seedlings of Boechera, and may be important for the bypass of the meiotic pathway (Shah et al., 2016).

In tetraploids and hexaploids, the oxidative stress of prolonged photoperiods might be different. This could be due to altered photosynthetic electron transport capacities (Coate et al., 2013), or to altered secondary metabolite profiles in polyploids and hybrids (Orians, 2000). We speculate that lowered oxidative stress in polyploids might not be severe enough to induce sufficient double strand breaks that would be essential for a correct processing of meiosis (Keeney et al., 2014). Consequently, meiosis and megasporogenesis might be disturbed. Failure of megasporogenesis might release aposporous initial cell (AIC) development. Cellspecific transcriptome studies on aposporous Hieracium subg. Pilosella suggested that contact and cross-talk between AICs and functional megaspores could be the trigger for mitotic development of the former and degeneration of the latter (Juranić et al., 2018). We suppose a similar interaction of AICs and megaspores in the Ranunculus auricomus complex as they always occur together in close neighborhood, and we observed the presence of AICs together with young (2-nucleate stage) meiotic embryo sacs but not at later stages (Suppl. Fig. $2 \mathrm{c}-\mathrm{d}$ and 4). The emergence of aposporous initials starts in the Ranunculus auricomus complex mostly at the end of megasporogenesis and is correlated to disturbance of megasporogenesis. The surviving aposporous cells grow faster than the meiotic cell and occupy the mode of megagametogenesis and seed development (Hojsgaard et al., 2014a; Barke et al., 2018). The stress only affects the megaspore but leaves apomeiosis as the surrogate for the sexual pathway (Hörandl and Hadacek, 2013). Alternatively, polyploids with more DNA content have more repair templates for the DSBs, and a higher dose of stress would be required to break the DNA (Schoenfelder and Fox, 2015). Here polyploidy might promote DNA damage tolerance under elevated stress as described (Schoenfelder and Fox, 2015) and buffers stress effects (Hörandl and Hadacek, 2013). 
Environmental stress plays a role as an inhibition factor under an epigenetic mechanism that disturbs or interrupts the silencing signal of apomictic-conditioning (Rodrigo et al., 2017). At least in diploid Ranunculus, the treatment might strengthen a signal transduction pathway that promotes switching from apomeiosis to meiosis, as demonstrated in facultative Boechera after drought stress (Mateo de Arias, 2015; Gao, 2018; Carman et al., 2019). In polyploids, the whole duplication genome (WDG) provides the co-loss or co-retention condition, which maintains a constant set of miRNA for basic biological functions (Liu and Sun, 2019). Our data suggested that polyploids respond to the stress via homeostatic regulation in the frequency of apospory vs. megasporogenesis. The high variability of the proportions of sexual ovules among our genetically identical polyploids supports the findings of epigenetic and transcriptional control mechanisms as the background for the phenotypic expression of apospory (Schmidt et al., 2014). Our result supports the hypothesis that phenotypic features of apomixis in flowering plants are strongly affected by polyploidy (Delgado et al., 2016; Kaushal et al., 2018) and subjected to epigenetic control (Rodrigo et al., 2017).

\subsubsection{Effects of ploidy, treatment, and combined effect of ploidy/treatment to the seed development and mode of reproduction}

The prolonged photoperiod affected neither the frequency of seed set, the proportion of sexual seeds, nor the pollen viability. Ranunculus auricomus complex plants generally lose a high seed proportion compared to rates of ovule formation due to their high seed abortion rate, exceeding one-half to two-third (Izmaiłow, 1996; Hörandl, 2008; Hörandl and Temsch, 2009; Klatt et al., 2016; Barke et al., 2018). This failure on seed formation arises at early stages and during the development of endosperm tissue (Barke et al., 2018). The diploid cytotype, which generally reproduces sexually, delivers a better seed set than the higher ploidy levels. In contrast, tetraploids and hexaploids, which are predominantly facultative apomictic, showed a reversed pattern, by increasing frequencies of asexual seeds.

Pollen quality is an external factor influencing the seed set of all cytotypes. The great variation in pollen quality, as observed here, is typical for apomictic plants (Asker and Jerling, 1992). The lower quality of tetraploid pollen was concomitant with a lower seed set of the tetraploids, while the better pollen quality in diploids and hexaploids corresponded to a higher seed set in these cytotypes. For seed formation, the contribution of a male gamete to fertilize the central nuclei is the major requirement for proper endosperm development (Vinkenoog et al., 2003). The diploids keep their sexual ovules growing into sexual seeds in both treatments, while the survival of three apomictic seeds in the stress treatment represented rare exceptions from seed abortion. Similar results have been reported from the garden experiment (Barke et al., 2018). Diploid plants are sensitive to genomic imprinting deviation in the endosperm (Hörandl 
and Temsch, 2009; Barke et al., 2018), i.e. a 2:1 constant ratio for maternal ( $\mathrm{m}$ ) to paternal (p) genome contribution to endosperm (Spielman et al., 2003; Vinkenoog et al., 2003; Hörandl and Temsch, 2009; Barke et al., 2018). The occurrences of genome imbalance in pseudogamously ( $4 \mathrm{~m}: 1 \mathrm{p}$ and $4 \mathrm{~m}: 2 \mathrm{p}$ ) and autonomously formed seed ( $4 \mathrm{~m}: 0 \mathrm{p})$ suggested that endosperm imbalance inhibited apomictic seed formation in our diploid cytotype.

On the other hand, in polyploids, the development of sexual ovules aborted to a large extent and was replaced by aposporous initials that completed megagametogenesis. Apomictic seed formation in polyploids is mainly influenced by the competitive capacity of the unreduced embryo sac formation rather than by the light regime during megagametogenesis and seed development (Hojsgaard et al., 2013; Klatt et al., 2016; Hodač et al., 2019). The surviving aposporous initials continue to develop into aposporous embryo sacs, and seeds are formed mostly via parthenogenesis and pseudogamous apomixis. This mode of reproduction is indicated by the parthenogenetic embryo (an unreduced egg cell develops without male gamete fusion) and pseudogamous endosperm (two unreduced polar nuclei fuses with one or two male gametes). Parthenogenesis appears mostly in our asexual polyploid seeds as a significant factor promoting unreduced gametophytes against reduced one and seed formation (Hojsgaard and Hörandl, 2019). A significant number of B॥-hybrids in tetraploids were formed through fertilization of unreduced egg cells as partial apomixis, as it was also occasionally observed in other FCSS studies (e.g. Schinkel et al., 2016; Barke et al., 2018; Klatt et al., 2018). This $B_{\| I}$-hybrid had probably an extremely long period of egg cell receptivity in this cytotype as assumed in diploid Ranunculus (Barke et al., 2018). Additionally, pollenindependent seed development via autonomous apomixis was also a rare event in polyploids. Asexual seed formation via pseudogamy is predominant in most apomictic plants (Mogie, 1992) as observed in our polyploids. The most common developmental pathway, however, used both sperm nuclei, or the unreduced sperm nucleus, for fertilization of polar nuclei, and hence restored the optimal $2 \mathrm{~m}: 1 \mathrm{p}$ ratio in the endosperm; these pathways result in a peak index of 3.0 in flow cytometric seed screening and represent the major proportions of apomictic seeds in both tetraploids (92\%) and hexaploids (88\%), see data in Table 2.2. Unbalanced genome contributions were also observed. Even though the diploids are quite sensitive to genomic imprinting, the polyploids in Ranunculus are more relaxed as expected (Grimanelli et al., 2012; Quarin, 1999). The current theory suggests that epigenetic mutation in polyploids creates relaxation on genomic imprinting during endosperm development (Grimanelli et al., 1997; Quarin, 1999; Kaushal et al., 2018). This could be the reason of higher seed set in hexaploid than in tetraploid cytotypes, similar to in hexaploid Potentilla puberula that had higher seed set than the tetraploids (Dobeš et al., 2018). These findings suggest the presence of a buffer effect on genomic imprinting in polyploids. 
Our results suggest that the light regime only affects the proportion of sexual ovules, but the effect does not continue on the mode of seed formation. This finding supports the oxidative stress initiation hypothesis (Hörandl and Hadacek, 2013) that light stress affects only female meiosis, but has no relevance to further development. Polyploids express predominantly apospory, probably by improved mechanisms to buffer the abiotic stress, and are able to establish apomictic seed formation. These findings are in line with the general observation that apomixis mostly occurs in polyploid plants, despite the fact that the pathway can occur in diploids as well, albeit in much lower frequencies. Hence, stress resistance of polyploids may indirectly facilitate the establishment of apomixis, but is not necessarily essential for its expression, as proposed by Hojsgaard and Hörandl (2019).

\subsection{Conclusions}

Three cytotypes of facultative $R$. auricomus complex express the alternation of proportions of asexual ovules into more sexual ovules after prolonged photoperiod. We hypothesize that light stress increases ROS formation that triggers oxidative stress. The oxidative stress might stimulate the meiotic DNA repair system in the megaspore mother cell and suppresses mitotic division, resulting in sexual ovules. The effect of prolonged photoperiod on megasporogenesis was most pronounced in diploids; the lower effect of light stress in polyploids is probably as a consequence of higher stress resistance. In polyploids, high rates of seed abortion left a lower proportion of sexual seeds, whereas in diploids the sexual pathway is still predominant. Seed formation is not influenced by environmental stress conditions, but rather depending on proper endosperm formation. Our findings shed light on the predominance of apomixis occurrence in polyploid plants.

Data Availability Statement: The raw data are deposited at the data repository of the University of Göttingen https://data.goettingen-research-online.de/, under doi $\underline{10.3389 / f p l s .2020 .00104}$

Author Contributions: FU and EH designed research. FU performed research, analyzed and interpreted data. CC contributed to FCSS and microsatellite analysis. FU wrote the manuscript with contributions of EH.

Funding: This project was funded by The German Research Fund DFG (DFG Hörandl Ho 4395 4-1) to $\mathrm{EH}$ and by the Indonesia endowment fund for education, grant no. PRJ2369/LPDP.3/2016 to FU. 
Conflict of Interest: The authors declare that the research was conducted in the absence of any commercial or financial relationships that could be construed as a potential conflict of interest.

Acknowledgments: Silvia Friedrichs for nursing the plants; Birthe Barke for help with data interpretation; referees for valuable comments on the manuscript.

\section{Supplementary Material}

\section{Supplementary Figures}



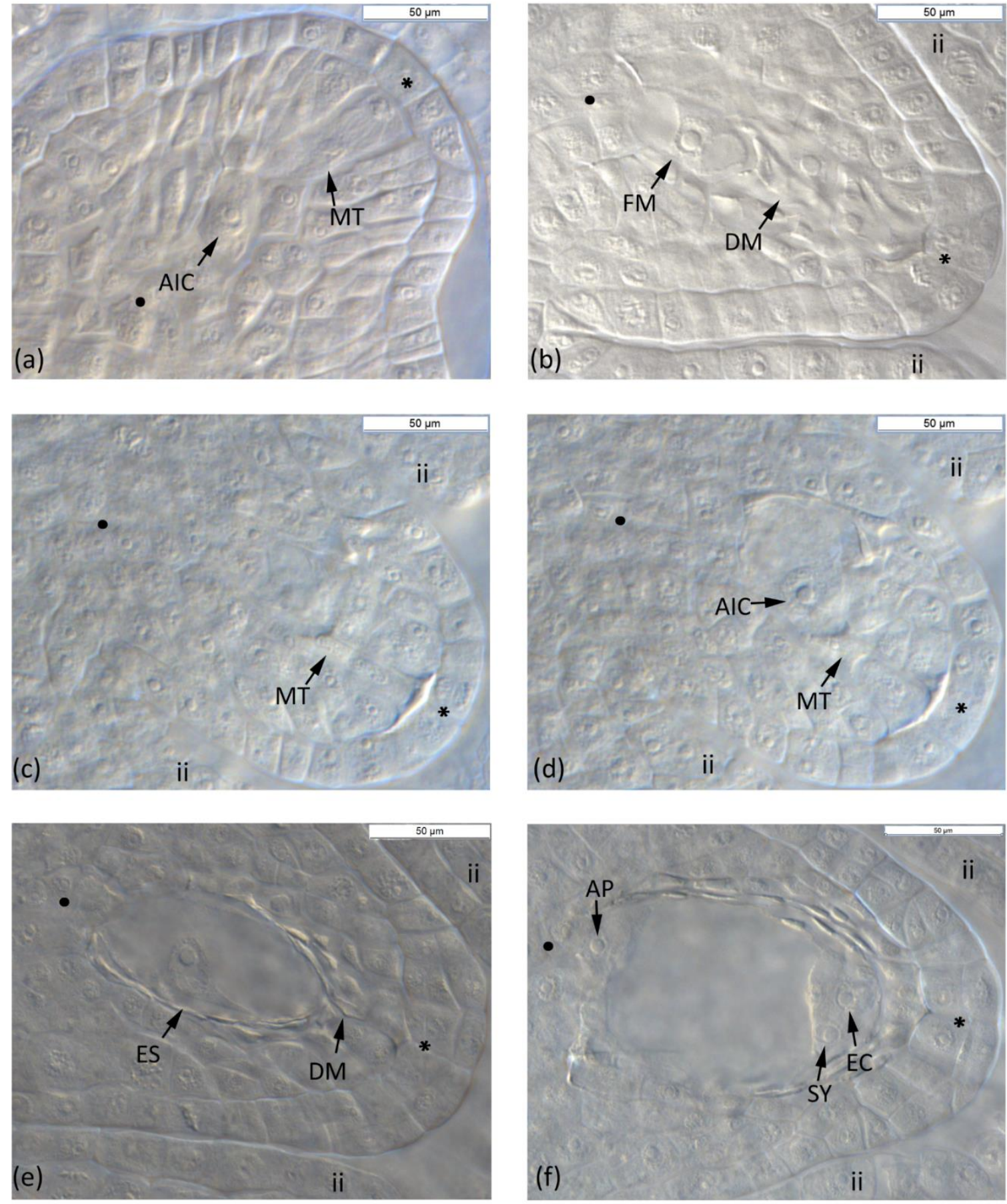

Supplementary Figure 1. Key reproductive stage of ovule development facultative apomictic diploid $R$. carpaticola $x$ notabilis. (a) Asexual ovule with megaspores in meiotic division and an aposporous initial cell; (b) Sexual ovule with a functional megaspore enlarge within two vacuoles and three aborted megaspore at micropylar pole; (c-d) One asexual ovule in two different layers, with an aborted megaspore tetrad (c) and a big aposporous initial cell (d); (e) Sexual ovule with a young embryo sac at germline position (one nucleus visible and another in another optical layer); (f) Mature embryo sac with egg cell and synergids at micropylar pole and antipodal cells at chalazal pole. Plant individual: (a) F3xJ6/25; (b) F10XF7/01; (c-d) F3xJ6/19; (e-f) J6XF3/23 . AIC, Aposporous Initial Cell; AP, Antipodal Cells; DM, degenerated megaspores; EC, Egg Cell; ES, Embryo Sac; FM, Functional Megaspore; ii, inner integument; MT, Megaspore Tetrad; SY, Synergid •, chalazal pole; *, micropylar pole. Scale bar: $50 \mu \mathrm{m}$. 

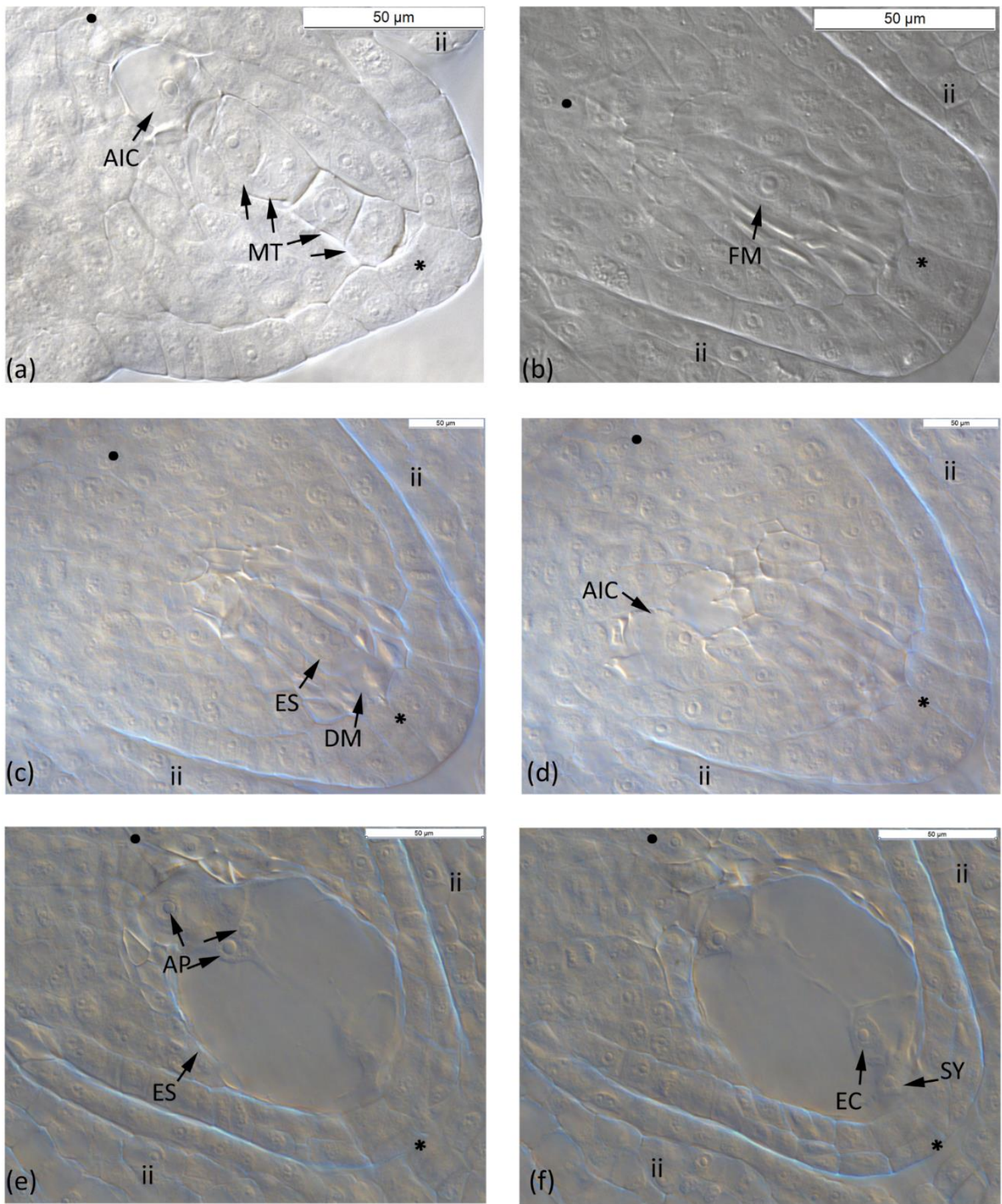

Supplementary Figure 2. Key reproductive stage of ovule development facultative apomictic tetraploid Ranunculus variabilis. (a) Asexual ovule with megaspore tetrads in alignment with an aposporous initial cell at chalazal pole; (b) Sexual ovule with an enlarged functional megaspore and degenerated meiotic products; (c-d) One asexual ovule in two different layers with a young (2-nucleate stage) meiotic embryo sac (c) and AIC (d); (e-f) One ovule in two different layers with a mature embryo sac with an egg cell and two synergids near to micropylar pole and three antipodal cells at chalazal pole. Plant individual: (a) LH1406030B4-7; (b) LH1406030B4-19; (c-d) LH1406030B5-08; (e-f) LH1406030B5-08. AIC, Aposporous Initial Cell; AP, Antipodal Cells; DM, degenerated megaspores; EC, Egg Cell; ES, Embryo Sac; FM, Functional Megaspore; ii, inner integument; MT, Megaspore Tetrad; SY, Synergid; $\bullet$, chalazal pole; ${ }^{*}$, micropylar pole. Scale bar: $50 \mu \mathrm{m}$. 

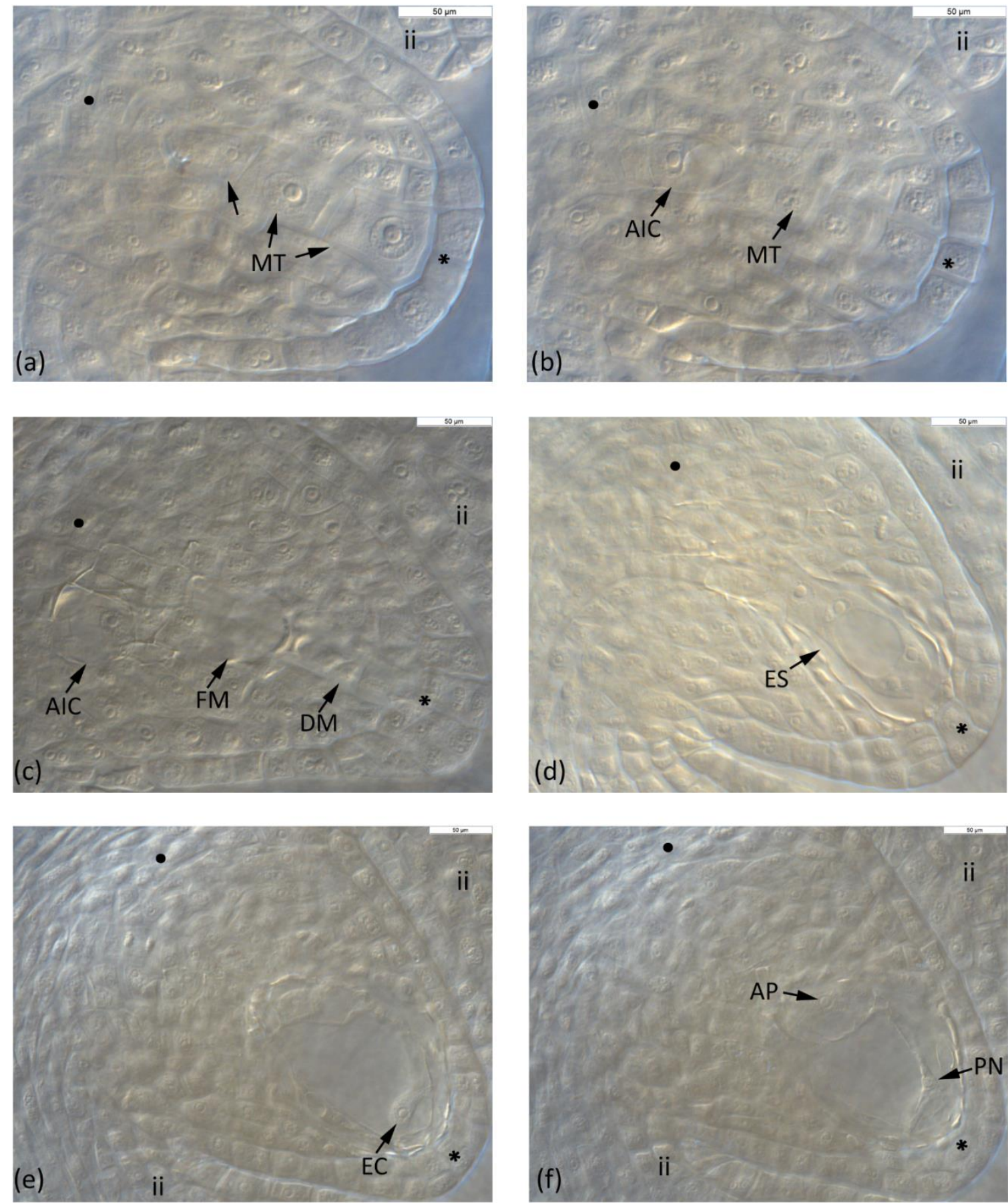

Supplementary Figure 3. Key reproductive stage of ovule development in facultative apomictic hexaploid Ranunculus carpaticola x cassubicifolius. (a-b) One asexual ovule in two different layers with megaspore tetrad cells in alignment (a) and an aposporous initial cell near chalazal pole (b); (c) Asexual ovule with functional megaspore and an AIC; (d) Ovule with young embryo sac after second nuclear division produced four nuclei; (e-f) One ovule in two different layers showing an embryo sac with an egg cell, polar nuclei, and Antipodal cells at chalazal pole; Plant individual: (a-b) 29/15-3V2/04; (c) 29/15-1L3/01; (d) 8492/6-2/04; (e-f) 29/15-3V2/27. AIC, Aposporous Initial Cell; AP, Antipodal Cells; DM, degenerating megaspores; EC, Egg Cell; ES, Embryo Sac; ii, inner integument; MT, Megaspore Tetrad; PN, Polar Nucleie, chalazal pole; *, micropylar pole. Scale bar: $50 \mu \mathrm{m}$. 

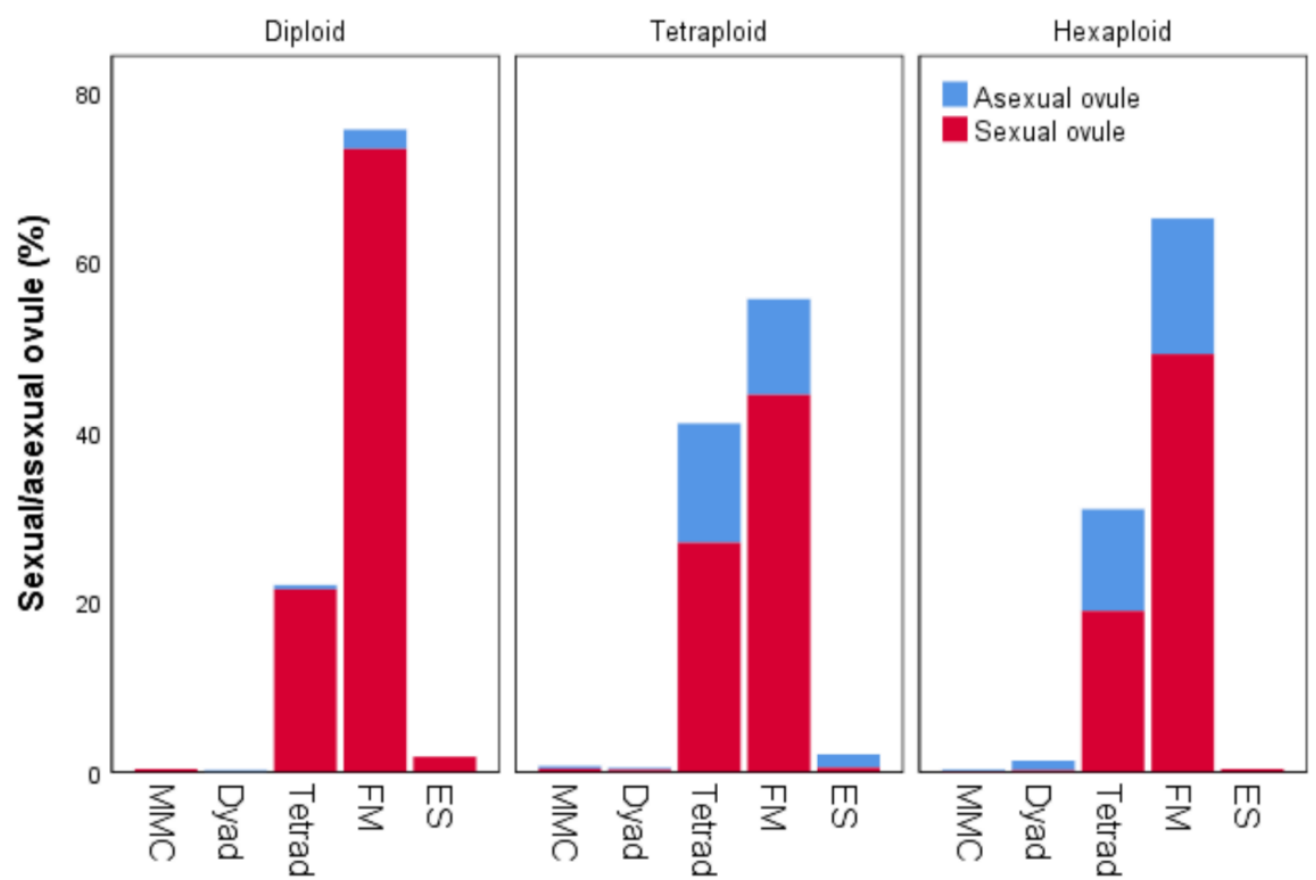

Supplementary Figure 4: Mean proportions of sexual and asexual ovules from each developmental stage in three cytotypes of the $R$. auricomus complex (both treatments pooled). MMC, Megaspore Mother Cell; Dyad, first meiotic product; Tetrad, completed meiosis has produced four megaspores; FM, Functional Megaspore (only the chalazal megaspore developed while the other three cells aborted); ES, young embryo sac. 


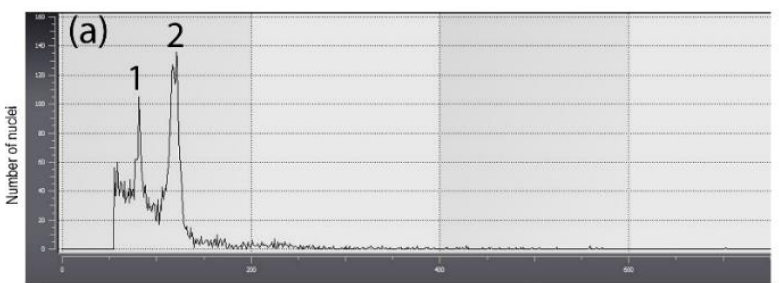

Fluorescence intensity
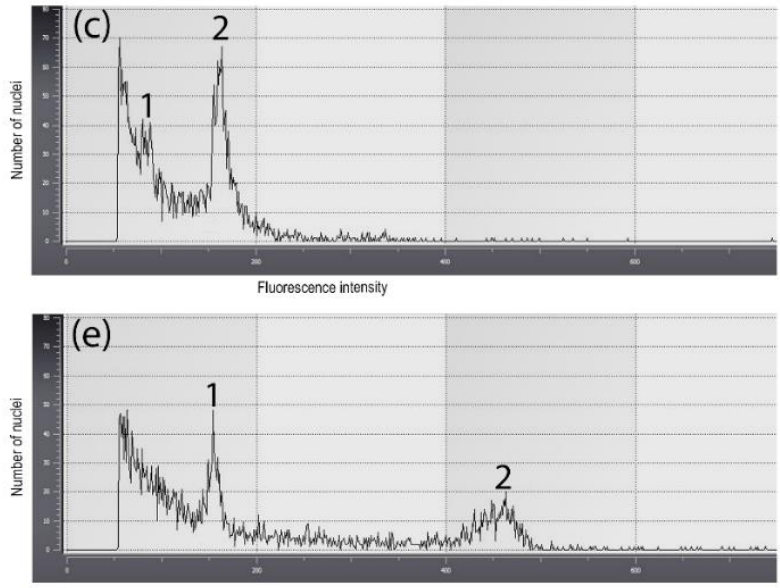

Fluorescence intensity

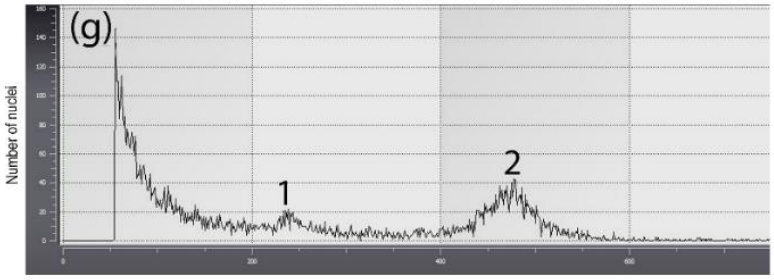

Fluorescence intensity

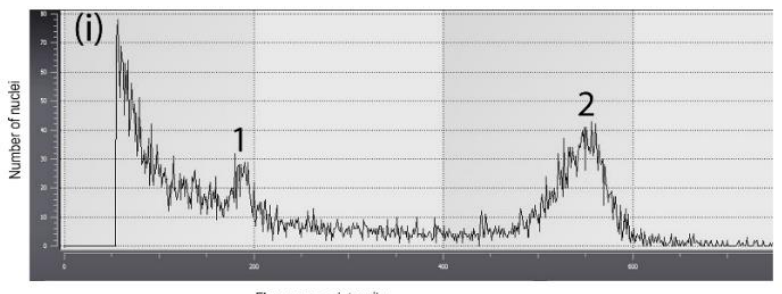

Fluorescence intensity

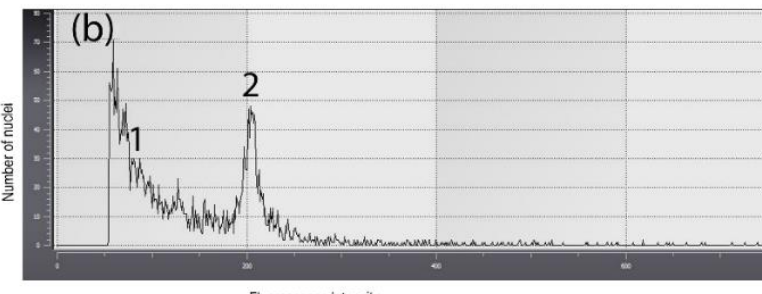

Fluorescence intensity
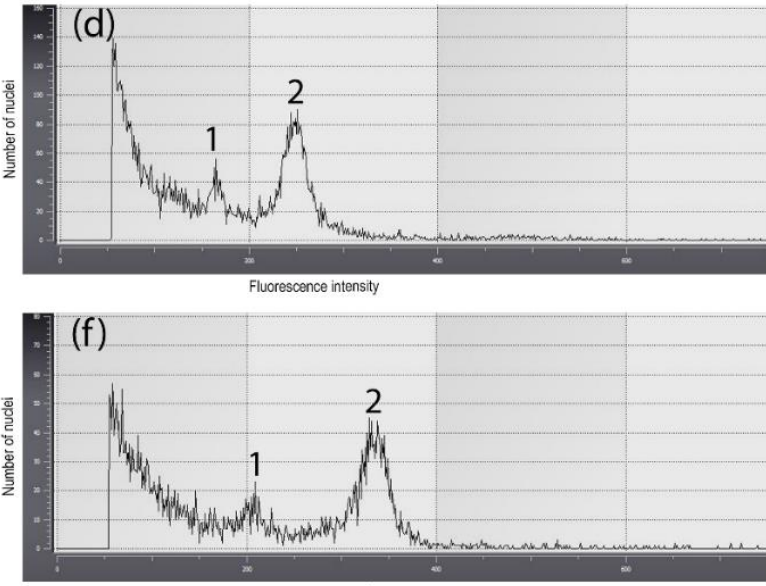

Fluorescence intensity

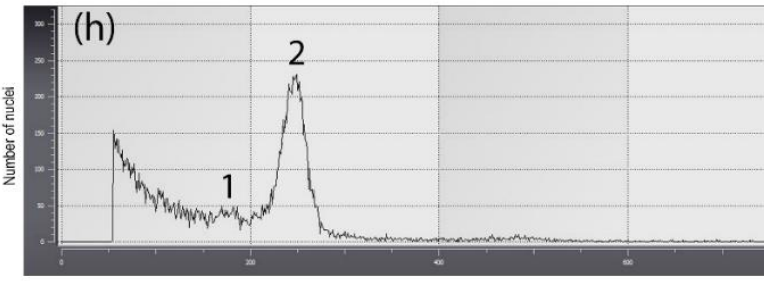

Fluorescence intensity

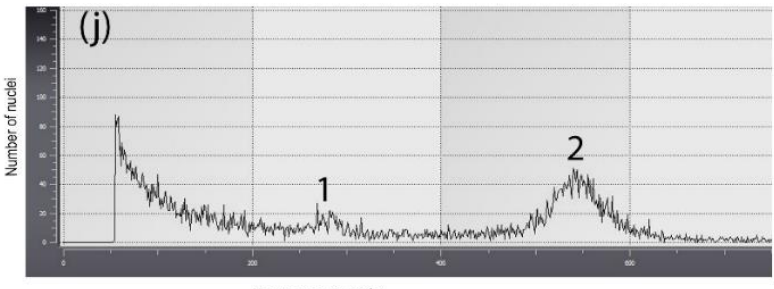

Fluorescence intensity

Supplementary Figure 5. Flow cytometry histograms of the Ranunculus auricomus complex. (a) diploid sexual seed; (b) diploid pseudogamous apomictic; (c) diploid autonomous apomictic; (d) tetraploid sexual; (e) tetraploid pseudogamous apomictic; (f-g) tetraploid BIII_hybrid; (h) hexaploid sexual; (i) hexaploid pseudogamous apomictic; (j) hexaploid BIII_hybrid. General peak labelling: 1 embryo peak, 2 endosperm peak. Plant individual: (a) J20xJ2/22; (b) F10xJ33/9; (c) F3xJ6/05; (d) LH1406030G1-16; (e) LH1406030B4-01; (f) LH1406030B2-07; (g) LH1406030B1-02; (h-i) 29/15$5 \mathrm{~K} / 31 ;$ (j) 29/15-6J/02. 


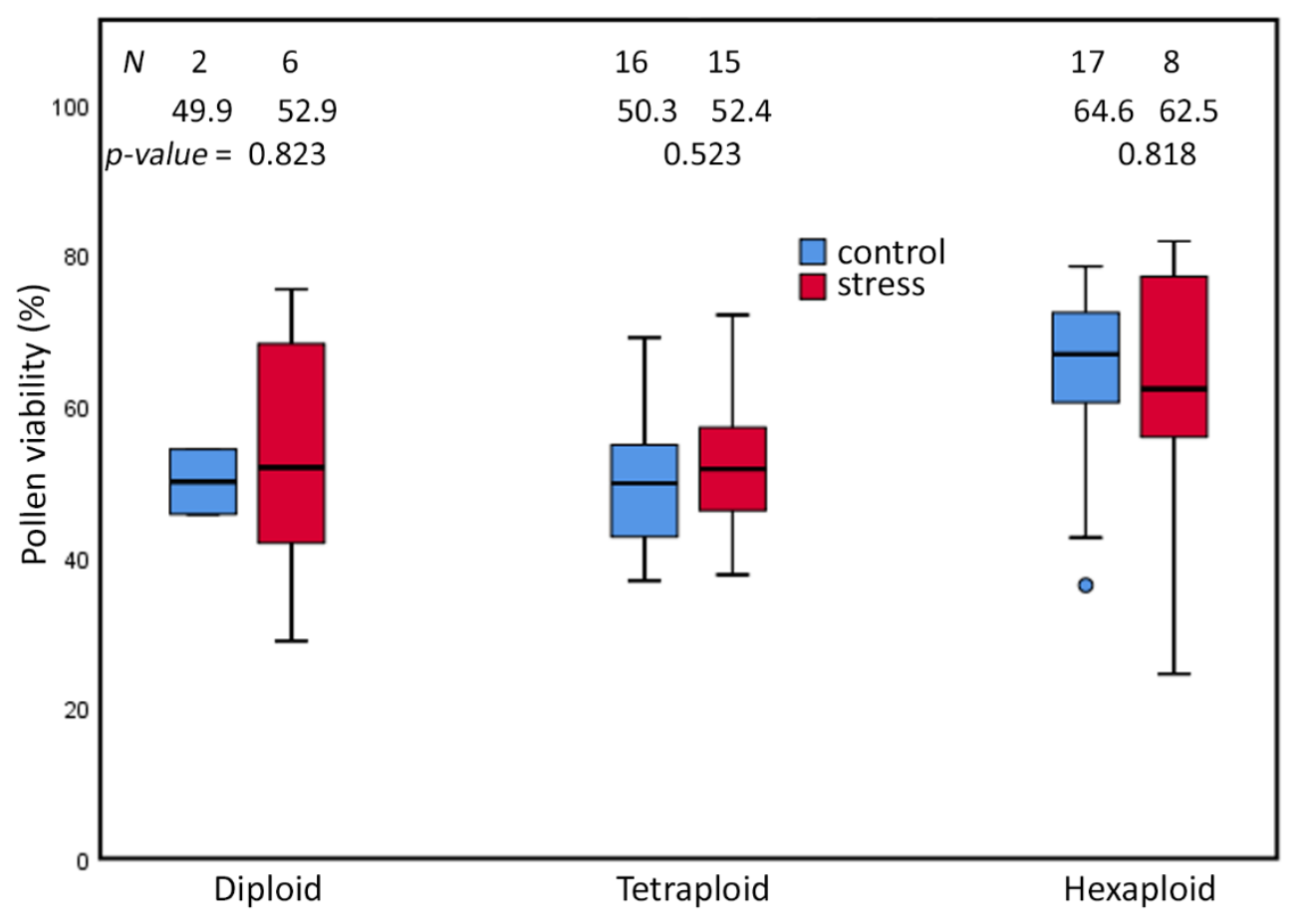

Supplementary Figure 6 . Proportions of viable pollen in the $R$. auricomus complex plants grown in climatic chamber under prolonged photoperiod (stress) and shortened photoperiod (control). Mean values and statistical significance are given in figure. $\mathrm{N}=$ number of individuals. For the test statistic, see Suppl. Table S2. 


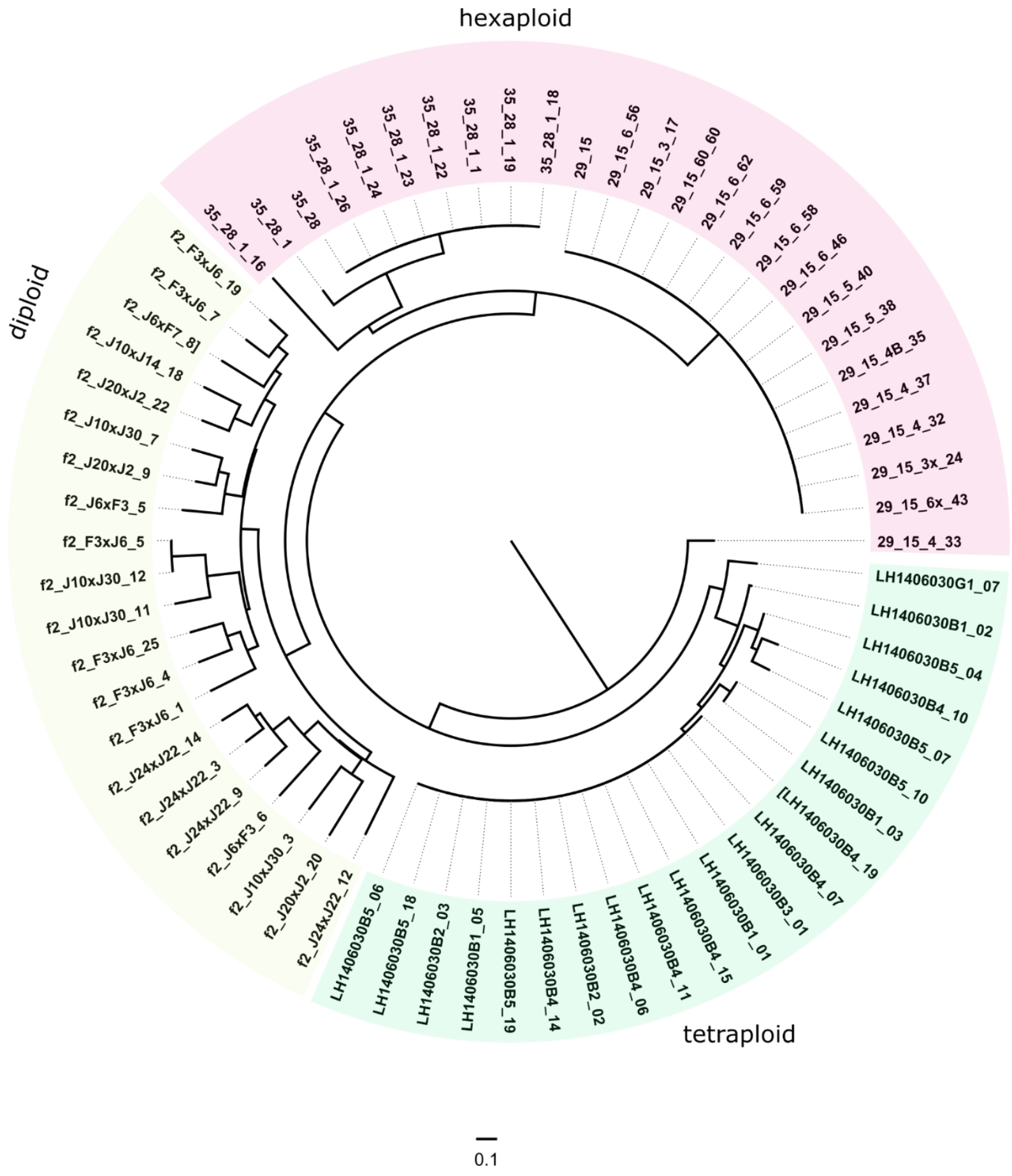

Supplementary Figure 7. Neighbor-joining tree derived from SSR data. Ranunculus auricomus complex from three different cytotypes and their clonal progeny were analyzed. Scale bar $=$ no. of changes. 


\section{Supplementary Tables}

Supplementary Table 1. Plant material list of Ranunculus auricomus complex from three different cytotypes. * indicated the plants used in different treatment in 2017 and 2018

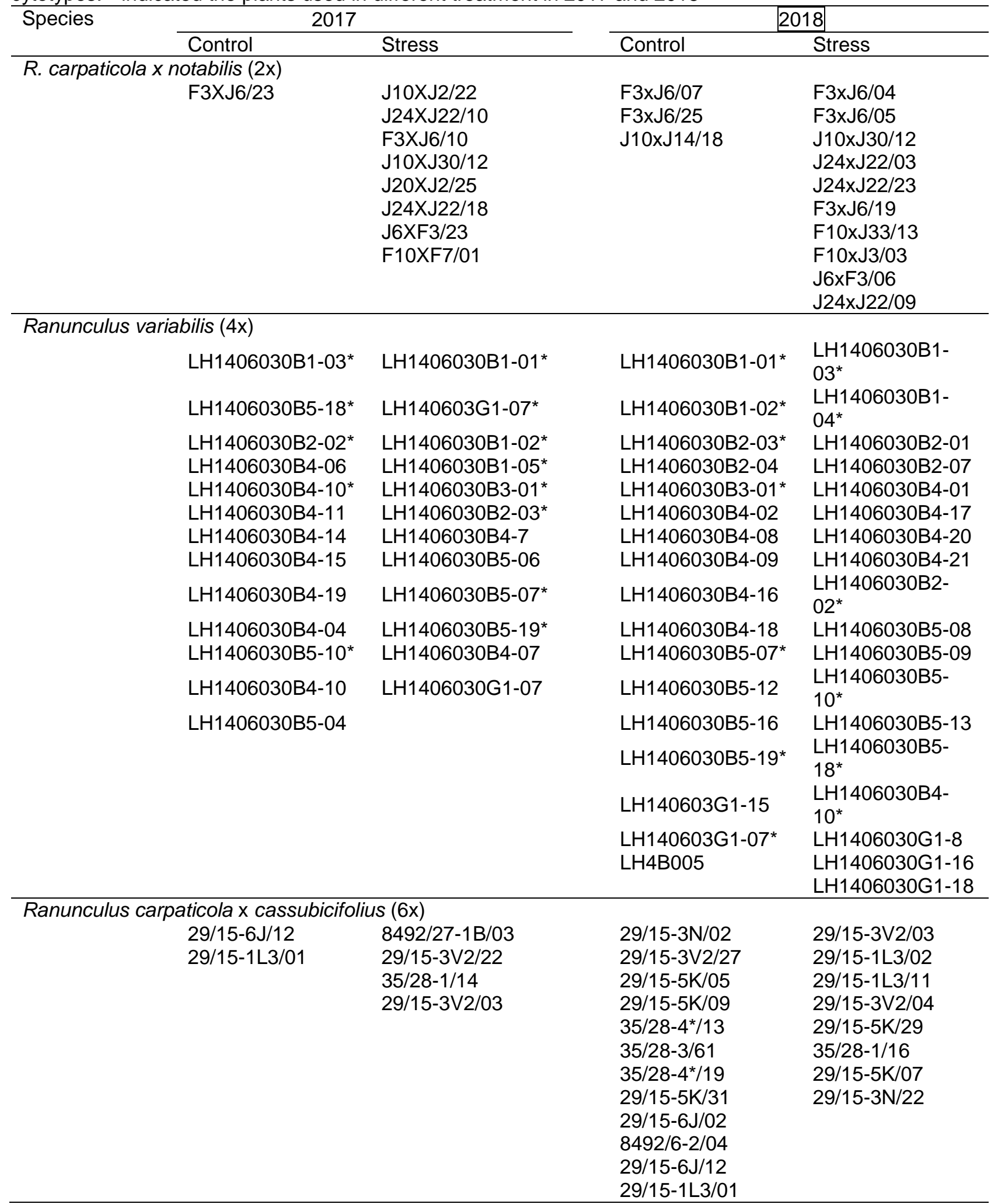


Supplementary Table 2. Statistical characteristic of the effect of light extension on the reproductive mode among ploidies of Ranunculus auricomus complex plants. P-values in bold indicate significances between light treatment.

\begin{tabular}{|c|c|c|c|c|c|c|}
\hline & \multicolumn{2}{|l|}{ Diploid } & \multicolumn{2}{|c|}{ Tetraploid } & \multicolumn{2}{|c|}{ Hexaploid } \\
\hline & Control & Stress & Control & Stress & Control & Stress \\
\hline & \multicolumn{6}{|c|}{ Proportion of sexual ovule } \\
\hline Median & 80.37 & 99.26 & 57.90 & 80.29 & 52.61 & 70.36 \\
\hline Mean & 80.37 & 100.00 & 58.97 & 84.34 & 56.94 & 80.00 \\
\hline SD & 19.38 & 1.26 & 8.79 & 10.67 & 26.11 & 20.04 \\
\hline $\mathrm{N}$ & 2 & 13 & 16 & 13 & 26 & 26 \\
\hline \multirow[t]{2}{*}{$p$-value } & 0.001 & & 0.001 & & 0.006 & \\
\hline & \multicolumn{6}{|l|}{ Seed-set } \\
\hline Median & 35.09 & 50.00 & 29.02 & 29.14 & 43.40 & 43.59 \\
\hline Mean & 39.84 & 50.22 & 31.09 & 28.97 & 42.17 & 43.04 \\
\hline SD & 23.02 & 14.89 & 9.66 & 7.75 & 12.65 & 10.92 \\
\hline $\mathrm{N}$ & 5 & 16 & 17 & 20 & 16 & 9 \\
\hline \multirow[t]{2}{*}{$p$-value } & 0.300 & & 0.459 & & 0.880 & \\
\hline & \multicolumn{6}{|c|}{ Reproduction mode } \\
\hline Median & 100.00 & 100.00 & 2.50 & 0.00 & 10.00 & 3.60 \\
\hline Mean & 100.00 & 98.74 & 6.59 & 3.88 & 9.66 & 5.08 \\
\hline & 0.00 & 3.30 & 8.27 & 4.75 & 11.15 & 5.32 \\
\hline & 5 & 15 & 17 & 18 & 13 & 8 \\
\hline \multirow[t]{2}{*}{$p$-value } & 0.337 & & 0.251 & & 0.293 & \\
\hline & \multicolumn{6}{|c|}{ Pollen viability } \\
\hline Median & 49.90 & 51.80 & 49.70 & 51.60 & 66.94 & 62.20 \\
\hline Mean & 49.90 & 52.97 & 50.33 & 52.44 & 64.62 & 60.70 \\
\hline & 6.08 & 17.38 & 9.22 & 9.51 & 11.73 & 19.95 \\
\hline & 2 & 6 & 16 & 15 & 17 & 11 \\
\hline$p$-value & 0.777 & & 0.536 & & 0.605 & \\
\hline
\end{tabular}


Supplementary Table 3. Pairwise comparison with Tukey HSD tests were conducted to determine the simple main effect of photoperiod on the proportion of sexual ovules among ploidies.

\begin{tabular}{|c|c|c|c|c|c|c|c|}
\hline \multirow[t]{2}{*}{ Treatment } & \multirow[t]{2}{*}{ Ploidy (I) } & \multirow[t]{2}{*}{ Ploidy (J) } & \multirow{2}{*}{$\begin{array}{l}\text { Mean } \\
\text { Difference (I-J) }\end{array}$} & \multirow[t]{2}{*}{ Std. Error } & \multirow[t]{2}{*}{ p-value } & \multicolumn{2}{|c|}{ 95\% Confidence Interval } \\
\hline & & & & & & Lower Bound & Upper Bound \\
\hline \multirow[t]{3}{*}{ Stress } & Diploid & Tetraploid & $0.554^{*}$ & 0.094 & $0.000^{*}$ & 0.366 & 0.743 \\
\hline & Diploid & Hexaploid & $0.677^{*}$ & 0.081 & $0.000^{*}$ & 0.514 & 0.84 \\
\hline & Tetraploid & Hexaploid & 0.123 & 0.081 & 0.137 & -0.040 & 0.286 \\
\hline \multirow[t]{3}{*}{ Control } & Diploid & Tetraploid & 0.356 & 0.198 & 0.08 & -0.044 & 0.756 \\
\hline & Diploid & Hexaploid & $0.396^{*}$ & 0.194 & $0.047^{*}$ & 0.005 & 0.788 \\
\hline & Tetraploid & Hexaploid & 0.040 & 0.084 & 0.633 & -0.129 & 0.21 \\
\hline
\end{tabular}

Based on observed means.

* The mean difference is significant at the 0.05 level. 
Supplementary Table 4. Two-way ANOVAs were conducted to determine the interaction effect of photoperiod and ploidy level on the proportion of well-developed seeds.

\begin{tabular}{llllll}
\multicolumn{1}{c}{ Source } & Type III Sum of Squares & df & Mean Square & F & p-value \\
\hline Ploidy & 0.445 & 2 & 0.222 & 11.167 & 0.000 \\
Treatment & 0.018 & 1 & 0.018 & 0.887 & 0.349 \\
Ploidy x Treatment & 0.050 & 2 & 0.025 & 1.246 & 0.293 \\
\hline
\end{tabular}

a. $R$ Squared $=0.307$ (Adjusted R Squared $=0.262$ ) 
Supplementary Table 5. Multiple comparisons with Tukey HSD tests were conducted to determine the simple main effect of photoperiod on the proportion of well-developed seeds among ploidies

\begin{tabular}{lllllll}
\hline Ploidy (I) & Ploidy (J) & $\begin{array}{l}\text { Mean } \\
\text { Difference (I-J) }\end{array}$ & Std. Error & p-value & \multicolumn{2}{c}{ 95\% Confidence Interval } \\
\cline { 5 - 7 } & & & & Lower Bound & Upper Bound \\
\hline Diploid & Tetraploid & $0.173^{*}$ & 0.043 & 0.000 & 0.088 & 0.259 \\
Diploid & Hexaploid & 0.036 & 0.047 & 0.445 & -0.057 & 0.129 \\
Tetraploid & Hexaploid & $-0.138^{*}$ & 0.037 & 0.000 & -0.212 & -0.063 \\
\hline
\end{tabular}

Based on observed means.

${ }^{*}$ The mean difference is significant at the 0.05 level. 
Supplementary Table 6. P-values for the Mann-Whitney U-test were conducted to determine the interaction effect of photoperiod and ploidy level on the proportion of sexual seeds.

\begin{tabular}{llll}
\hline & Diploid & Tetraploid & Hexaploid \\
\hline Mann-Whitney U & 30 & 118.5 & 47 \\
Wilcoxon W & 150 & 271.5 & 83 \\
Z & -1.053 & -0.678 & -0.366 \\
Asymp. Sig. (2-tailed) & 0.292 & 0.497 & 0.714 \\
Exact Sig. [2*(1-tailed Sig.)] & $0.553 \mathrm{c}$ & 0.533 & 0.750 \\
\hline
\end{tabular}


Supplementary Table 7. Multiple comparisons with Tukey HSD tests were conducted to determine the simple main effect of photoperiod on the proportion of viable pollen among ploidies

\begin{tabular}{lllllll}
\multicolumn{2}{l}{ Ploidy (I) } & Ploidy (J) & $\begin{array}{l}\text { Mean } \\
\text { Difference (I-J) }\end{array}$ & Std. Error & p-value & \multicolumn{2}{l}{$\mathbf{9 5 \%}$ Confidence Interval } \\
\cline { 5 - 7 } & & & & Lower Bound & Upper Bound \\
\hline Diploid & Tetraploid & 0.015 & 0.063 & 0.968 & -0.136 & 0.167 \\
Diploid & Hexaploid & -0.015 & 0.063 & 0.968 & -0.167 & 0.136 \\
Tetraploid & Hexaploid & $-0.152^{*}$ & 0.042 & 0.001 & -0.253 & -0.053 \\
\hline
\end{tabular}

Based on observed means.

${ }^{*}$ The mean difference is significant at the 0.05 level. 
Chapter 3: Ploidy-dependent effects of prolonged photoperiod on photosynthesis performance of the Ranunculus auricomus complex (Ranunculaceae)

Fuad Bahrul Ulum, Franz Hadacek and Elvira Hörandl

In prep to submit to Biology

\subsection{Simple Summary}

Polyploidy enhances stress-tolerance compared to their diploid progenitors. Prolonged photoperiod in plants influences flowering, photosynthesis, growth, and metabolite profiles. A previous study suggested ploidy-dependent effects of photoperiod on mode of reproduction in three cytotypes of the Ranunculus auricomus complex. In this study we investigated the ploidy-dependent effect of photosynthesis performance under different photoperiods. Among cytotypes, diploids were more sensitive to the extended photoperiod compared to polyploids. In tetraploids, the fraction of excess light was quenched into photochemistry, but another fraction exceeded the capacity of photon trapping, hence dissipated as non-photochemical quenching. The hexaploids presented a high variation of photosynthesis performance among two clones which might relate to different ecotypes. Photosynthesis performance of $R$. auricomus relates to the mode of ovule formation, as diploids showed the highest sensitivity to prolonged photoperiod concomitant to the highest proportions of sexual ovules, followed by tetraploids. Hexaploids, however, exhibited a very large variance in the proportions of sexual ovules, which we also observed here in photosynthesis performance

\subsection{Abstract}

Polyploidy (whole-genome duplication), enhances tolerance to drastic environmental stress compared to their diploid progenitor by diversifying genetic regulatory mechanisms. Prolongation of photoperiod influences flowering, photosynthesis, growth, and metabolite profiles of plants. Among plant organs, the reproductive part is most sensitive to stress. Light stress creates photodamage due to the inhibition of photosystem II (PSII) repair and alteration in the photosynthetic redox signaling pathways. Following a previous study on the ploidydependent effect of photoperiod on the female reproductive organs in three cytotypes of the Ranunculus auricomus complex, we investigated the possible role of polyploidy in photosynthesis performance by applying an extensive analysis of chlorophyll a fluorescence. We examined photosynthesis activity based on the parameters maximum efficiency of PSII ( $\phi P S I I)$, maximum quantum yield (QY_max), relative electron transport rate (rETR), nonphotochemical quenching (NPQ), photochemical quenching (PQ), quenching coefficients, and fast fluorescence transient (OJIP) curves on the first basal leaves, which appear 
synchronously with flower buds. We applied different photoperiods (12 hours and 16.5 hours) in two climate growth chambers and kept all other conditions equal (light intensity, temperature, and humidity). We hypothesized that prolonged photoperiod would affect photosynthetic efficiencies and hence redox homeostasis differentially in the three cytotypes. The results of rETR, PSII, and QY_max indicated the light intensity of the climatic chamber was sufficient for photosynthesis. Among cytotypes, diploids were more sensitive to the extended photoperiod compared to polyploids as indicated by the alternation of nonphotochemical quenching parameters (NPQ, $q_{E}, N P Q_{E}$, and $q N$ ), specific energy flux parameters ( $A B S / R C, D_{0} / R C$, and $\left.T R_{0} / R C\right)$, and performance index on absorption basis (PI_Abs). In tetraploids, the fraction of light excess was quenched into photochemistry $(q P)$, but another fraction exceeded the capacity of photon trapping $\left(T R_{0} / R C\right)$, hence dissipated as non-photochemical quenching $(q L)$. The hexaploids presented a high variation of photosynthesis performance among two clones in all parameters, which might relate to their origins from different habitats. However, the photosynthesis performance of $R$. auricomus relates to the mode of ovule formation, as diploids showed the highest sensitivity to prolonged photoperiod concomitant to the highest proportions of sexual ovules, followed by tetraploids. Hexaploids, however, exhibited a very large variance in the proportions of sexual ovules, which we also observed here in photosynthesis performance. We detected here that this variation is mostly referable to two different ecotypes, supporting a hypothesis of a higher flexibility of high polyploids in their stress response.

Keywords: Cytotype, photoperiod, photochemistry, photosynthesis, polyploidy, quenching, Ranunculus

\subsection{Introduction}

Polyploidy, whole-genome duplication, is the presence of double or multiple chromosome sets by either genome doubling in a single species (autopolyploidy) or hybridization of two species with associated genome doubling (allopolyploidy) (Landis et al., 2018). Polyploidy enhances stress tolerance in response to drastic environmental change by enabling more extensive adaptations as consequences of gene and genome duplication (Van de Peer et al., 2020) and acts as drivers of evolution and speciation in plants (Alix et al., 2017). Compared to their diploid progenitor, polyploids exhibit a better stress resistance, e.g., an increase of the ABA signaling pathway under drought stress (Rao et al., 2020) or cold stress (Wu et al., 2004). Polyploid plants alter metabolite profiles and photosynthesis under cold stress (Lourkisti et al., 2020), increase xanthophyll pigment (Coate et al., 2013), and show a greater antioxidant activity under light stress (Oustric et al., 2018). Polyploid plants perform photosynthesis more efficiently (Warner and Edwards, 1993; Oustric et al., 2018) because they have larger 
mesophyll cells containing more chloroplasts, higher chlorophyll content, and higher contents of the enzyme RuBisCo (Ribulose-1,5-bisphosphate-carboxylase/-oxygenase) than their diploid relatives (Coate et al., 2012; Münzbergová and Haisel, 2019). Moreover, the diversity of genomes, transcriptomes, and metabolomes of polyploids increases their resistance to environmental stress (Schoenfelder and Fox, 2015). Alternation of metabolite profiles in polyploids also has been reported (lannicelli et al., 2020).

Photoperiod is the daily illumination received by an organism, naturally promoted by the tilt of the earth's axis (Jackson, 2009). In plants, photoperiod extension influences flowering (Jeong and Clark, 2005; Jackson, 2009), photosynthesis (Bauerle et al., 2012; Kinoshita et al., 2020), growth (Wu et al., 2004), metabolite profiles (Sulpice et al., 2014; de Castro et al., 2019), and production of reactive oxygen species (ROS) (Abuelsoud et al., 2020). Bauerle et al. (2012) reported that photosynthesis was more substantially associated with photoperiod than temperature regime in 23 tree species. Several studies addressed stress effects of photoperiod, e.g., modification of leaf structure and metabolism of Ginkgo biloba (Kinoshita et al., 2020) and destruction of flower meristem development (Jeong and Clark, 2005). In Arabidopsis, photoperiod altered trehalose 6-phosphate and amino acid biosynthesis intermediate shikimate as key coordinators for growth rate (Sulpice et al., 2014).

Light stress in plants occurs whenever absorption of environmental illumination in the leaves is higher than energy use and cannot be safely dissipated (Müller et al., 2001). Under natural conditions, plant exposure to the sunlight severed unfavorable risk to the photosynthetic component due to the formation of reactive oxygen species (ROS) as a by-product of light excess (Demmig-Adams and Adams III, 2006). This light stress creates photodamage due to the inhibition of photosystem II (PSII) repair and alternation in the photosynthetic redox signaling pathways (Gururani et al., 2015). When the photodamage rate exceeds the repair rate, PSII is downregulated, and this condition is called photoinhibition (Vass, 2012). Plants can escape this situation by applying regulation for the repartition of absorbed light between photochemistry and energy dissipating pathways as a photoprotective mechanism (Lavaud, 2007). The photoprotection accommodates light residue via leaf and chloroplast, modification of light-harvesting antenna, scavenging of ROS, and regulate thermal dissipation (Murchie and Niyogi, 2011). Among plant organs, the reproductive part is most sensitive to stress, e.g., reduction of seed set appeared under moderate temperature stress (Sato et al., 2006). Destructive effects on male and female development were reported by influenced of water, temperature, light, and oxidative stress review in Ma et al. (2020).

The expression of apomixis, i.e., the asexual reproduction via seeds (Asker and Jerling, 1992), is influenced by stress. Frequencies of asexual reproduction in facultative apomictic plants 
were altered after stress, e.g., in Boechera (Mateo de Arias, 2015), Ranunculus (Klatt et al., 2016; Ulum et al., 2020), Eragrostis (Selva et al., 2020), and Paspalum (Karunarathne et al., 2020). Apomeiosis, the production of unreduced embryo sacs, is the key developmental step in gametophytic apomixis, the type that is relevant here (Asker and Jerling, 1992; Hodač et al., 2014). Apomeiotic versus meiotic development is influenced by ROS scavenging (Mateo de Arias et al., 2020). In Arabidopsis, photoperiod stress induces ROS scavenging system associated with catalase reduction and an increase of apoplastic peroxidase (Abuelsoud et al., 2020). The excess of ROS in reproductive tissue generates oxidative stress (Stangherlin and Reddy, 2013; Milev and Reddy, 2015). In the archespor, oxidative stress might lead to DNA double strand-breaks and induction of meiosis as a DNA repair mechanism (Hörandl and Hadacek, 2013). Stress-adapted plants are able to maintain the metabolic network in ROS scavenging including compatible solutes, antioxidants and stress-responsive proteins (Obata and Fernie, 2012). Increase of ROS scavenging secondary metabolites, e.g., of flavonoids, are linked to the increase of ROS in reproductive tissue e.g. (Paupière et al., 2017). In polyploid plants, the higher stress tolerance reduces oxidative stress (Wu et al., 2004; Coate et al., 2013; Oustric et al., 2018; Lourkisti et al., 2020; Rao et al., 2020). Hence, in facultative apomictic polyploids, lowered stress levels could result in a decrease of proportions of meiotic ovules and favor apomeiotic development (Hörandl and Hadacek, 2013). This effect was as observed after different photoperiod treatments in three cytotypes of the Ranunculus auricomus complex (Klatt et al., 2016; Ulum et al., 2020). A recent study on the Ranunculus auricomus complex over a large geographical area in Europe revealed that among many climatic parameters, light intensity was positively correlated to the distribution of sexual reproduction (Karbstein et al., 2021). Based on these results we focus here on light treatments as the most effective natural abiotic stress factor in this group. However, so far, no experimental study is available to test effects of different photoperiods on photosynthesis performance on different cytotypes. This information is crucial to establish the hypothetical causal link between light stress and mode of reproduction.

The Ranunculus auricomus complex comprises diploid to tetraploid sexual species and polyploid facultative apomictic, aposporous, and pseudogamous lineages (Nogler, 1984b; Hodač et al., 2014; Karbstein et al., 2021). Three cytotypes were studied previously for testing the reproduction mode and their stress sensitivity to extended light (Klatt et al., 2016; Ulum et al., 2020). These studies presented a stress buffer effect of polyploidy on the formation of meiotic ovules. Our study aim was to investigate the possible role of polyploidy in the photosynthesis performance of $R$. auricomus complex by extending the photoperiod. Three cytotypes of $R$. auricomus complex (diploids, tetraploids, and hexaploids (Ulum et al., 2020) were exposed to prolonged photoperiods as moderate stress and compared to controls with 
short photoperiods. We examined the photosynthetic capacity under stress and control conditions by measuring chlorophyll fluorescence as an indicator of the photoprotective mechanism. The Chl a fluorescence is widely used for the analysis of photosynthesis performance in stressed plants (Maxwell and Johnson, 2000; Demmig-Adams and Adams III, 2006; Baker, 2008; Ptushenko et al., 2013). Measurement of Chl a fluorescence provided information on the efficiency of photochemical processes in photosystem II ("photochemical quenching") and heat dissipation of excess light ("non-photochemical quenching") (Maxwell and Johnson, 2000; Ptushenko et al., 2013; Lazár, 2015; Torres et al., 2021). In this study, we compare the parameters of PSII maximum efficiency ( $\phi P S I I)$, maximum quantum efficiency of PSII photochemistry (QY_max), relative electron transport rate (ETR), non-photochemical quenching (NPQ), photochemical quenching (PQ), quenching coefficients, and fast fluorescence transient curve (OJIP curve). We hypothesized that prolonged photoperiod would affect photosynthetic efficiencies and hence redox homeostasis differentially in the three cytotypes. A detailed study correlating photosynthesis data, metabolite profiles, and mode of the reproduction will be presented elsewhere.

\subsection{Material and Methods}

\subsubsection{Plant material and growth condition}

Ranunculus auricomus plants comprise the same individuals as in a previously published study (Ulum et al., 2020). Diploids comprised synthetic F2 hybrids crosses of the sexual taxa R. carpaticola x R. notabilis (Barke et al., 2018; Barke et al., 2020), tetraploid plants were grown from seeds of plants that were originally collected near Schönau, Mühlkreis, Austria (48 $22^{\prime} 46.00^{\prime \prime N} 14^{\circ} 44^{\prime} 46.00 " E$, wet meadow) by L. Hodač and K. Spitzer (LH002, GOET), and hexaploid plants were grown from seeds of natural hybrids of $R$. carpaticola $\times R$. cassubicifolius (original clone 29 from a forest margin and clone 35 from a meadow (Hörandl et al., 2009; Klatt et al., 2016). Materials are documented in Table S1. All seedlings were grown under equal conditions in the Old Botanical Garden of the University of Göttingen. At all ploidy levels, plants represented closely related hybrid genotypes (Ulum et al., 2020). Hexaploids showed great variance in all photosynthetic parameters and hence were grouped into two separate clones (clone 6x_29 and 6x_35) due to their distinctive photosynthesis performance (Table S1). Growth conditions for control and stress treatments have been optimized in a previous study (Klatt et al., 2016). The climatic chambers were set at a temperature of $18^{\circ} \mathrm{C}, 60 \%$ humidity, and an average light intensity of about $250 \mu \mathrm{mol}$ photons $\mathrm{m}^{-2} \mathrm{~s}^{-1}$. The photoperiod spanned $10 \mathrm{~h}$ for control and $16.5 \mathrm{~h}$ for light stress treatment (Klatt et al., 2016). 


\subsubsection{Photosynthesis}

We analyzed the effect of extended photoperiod on photosynthesis efficiency as a proxy of stress conditions. All photosynthesis analyses were performed on 3-11 plants per ploidy per treatment to a fully developed basal leaf (upper side) that supported the inflorescence. Measurement was started from the first weeks after sprouting of plants in March 2019 when plants produce flower buds. During this developmental step, the separation between meiotic versus apomeiotic development takes place in the ovules and hence is the stress-sensitive period (Hodač et al., 2014; Ulum et al., 2020). Flower buds are covered by green sepals as photosynthetic tissue, and hence we expect the same photosynthetic performance and stress effects as in the basal leaves.

The photosynthesis performances were observed by measurement of chlorophyll fluorescent intensity with a PAM fluorometer, PAR-FluorPen FP 110 (Photon Systems Instruments, Drásov, Czech Republic). Parameter formulae and explanations of photosynthesis efficiency were provided in Table S2. By using the preprogrammed device protocol, first, we measured the leaves without pre-dark adaptation to record the PSII maximum efficiency $\left(\phi P S \|=F^{\prime} v / F^{\prime}{ }_{M}\right)$. Then, plants were dark-adapted for at least 30 min for further parameter measurements, i.e., light curve to extract relative electron transport rate (rETR), fluorescence induction curve (IC) of non-photochemical quenching (NPQ), and fast fluorescence transient curve (OJIP curve). The maximum quantum efficiency of PSII photochemistry (QY_max $=F_{V} / F_{M}$ ) was extracted from all dark-adapted measurements (LC, IC, and JIP-test). QY_max values quantify the maximum photochemical capacity of open PSII complexes (Roháček et al., 2008).

\subsubsection{Relative electron transport rate}

rETR was assessed using light curve 1 (LC1). The measurement was started with an initial saturating super pulse in the dark, and subsequently, similar pulses were applied during an actinic light phase with increasing intensity, 10, 20, 50, 100, 300, and $500 \mu \mathrm{mol}$ photons $\mathrm{m}^{-2}$ $\mathrm{s}^{-1}$ (at photosynthesis photon flux density (PPFD)). Higher light intensities were not used due to the strong intensity decrease at $500 \mu \mathrm{mol}$ photons $\mathrm{m}^{-2} \mathrm{~s}^{-1}$ actinic light intensity. The measuring pulse was set to $0.09 \mu \mathrm{mol}$ photons $\mathrm{m}^{-2} \mathrm{~s}^{-1}$, the saturating and super pulses to $2400 \mu \mathrm{mol}$ photons $\mathrm{m}^{-2} \mathrm{~s}^{-1}$, and the actinic light to $300 \mu \mathrm{mol}$ photons $\mathrm{m}^{-2} \mathrm{~s}^{-1}$. QY max was extracted from the saturating pulse, and $\phi P S I$ values were extracted from the respective actinic light intensities. rETR was calculated with the equation ETR $=\phi P S I I x P P F D \times 0.5$ (Duarte et al., 2015). 


\subsubsection{Fluorescence quenching analysis of induction curve}

IC was attained using NPQ1 program. The measuring pulse was the same as in the light curve (LC1) analysis. After the initial saturating super pulse, actinic light was switched on and lasted for $1 \mathrm{~min}$. Within this time period, five additional super pulses were applied, the first one after $7 \mathrm{~s}$ and the following in a $12 \mathrm{~s}$ interval. The subsequent dark period lasted $88 \mathrm{~s}$, during which three super pulses were applied, the first one after $11 \mathrm{~s}$ and the following in a $26 \mathrm{~s}$ interval. From IC of each light phase, we extracted quenching coefficients of baseline (minimum) and maximum fluorescence and fluorescence at a certain time, i.e., in the first dark-adapted state: $F_{0}$ and $F_{M}$; in the light-adapted state: $F_{0}^{\prime}, F_{M}^{\prime}$, and $F(t)$; and in darkness: $F^{\prime \prime}{ }_{0}$ and $F^{\prime \prime} M$ (Supplementary Figure 1). Following (Lazár, 2015), a number of IC parameters were extracted from the quenching coefficient, i.e., total non-photochemical quenching (NPQ) and photochemical quenching (PQ); quenching analysis to discriminate types of NPQ, i.e., energy dependent non-photochemical Chl fluorescence quenching $\left(q_{E}\right)$, photoinhibition nonphotochemical Chl fluorescence quenching $\left(q_{l}\right)$, and NPQ parameter related to $q_{E}$ and $q_{1}$ $\left(N P Q_{E}\right.$ and $\left.N P Q_{1}\right)$; and energy partitioning under consideration of active centers only, i.e., quenching coefficient of photochemical $(q P)$, quenching coefficient of non-photochemical $(q M)$, and quenching coefficient of non-photochemical that is formed only upon illumination to regulate the amount of absorbed light in avoiding damage effect $(q L)$.

\subsubsection{Fast fluorescence transient}

OJIP curve followed the method of Strasser et al. (2000). The OJIP curve was induced by a pulse red light of $3000 \mu \mathrm{mol}$ photons $\mathrm{m}^{-2} \mathrm{~s}^{-1}$. The relative fluorescence intensity of the OJIP curve at points $\mathrm{O}, \mathrm{J}, \mathrm{I}, \mathrm{P}\left(\mathrm{F}_{0}, \mathrm{~F}_{\mathrm{J}}, \mathrm{F}_{\mathrm{I}}\right.$, and $\mathrm{F}_{\mathrm{M}}$, respectively) correspond to times of $0,2,30$, and $1000 \mathrm{~ms}$. Analysis of the change of relative variable fluorescence at point J, I, P was conducted with JIP-test parameters. Several OJIP measurements were taken from additional individuals due to the limited number of remaining basal leaves (Table S1).

\subsubsection{Statistical analysis}

All statistical analyses were conducted in $R$ Studio (version 1.0.153), running $R$ for windows (version 4.0.2, R Foundation for Statistical Computing). Data handling and visualization were performed using the dplyr, tidyr, purr, and ggplot2 package. Before all analyses, we checked the data's normality distribution by visual inspection of their frequency histograms and qq-plots using the ggpubr package. The photoperiods' effect on photosynthetic performance among different ploidies was tested using linear models $(\mathrm{Im})$ when the normality distribution assumptions were met. When the assumptions were not met, generalized linear mixed models 
with beta regression distribution were used or alternatively Kruskal-Wallis test for a lower sample number.

The $\phi P S I I$ and QY_max data (continuous variables with values ranging from 0 to 1 ) displayed left-skewed distributions and were calculated as a beta distribution. Therefore we run the analysis of $\phi$ PSII and QY_max with a generalized mixed model framework that can handle beta distribution (Ferrari and Cribari-Neto, 2004). We employed generalized linear mixed models (GLMMs) using the glmmTMB package (Brooks et al., 2017) to explore the effect of extended photoperiod on the $\phi P S I I$ and QY_max among ploidies. GLMMs analyzed the relationship between $\phi P S I I$ or $Q Y$ max as a response variable, ploidy level, photoperiod, and their interactions as predictor variables, and logit link function and a beta distribution as well. Plant ID was treated as a random effect in $\phi P S I I$ modeling, while the measurement origin (Data $=$ NPQ, ETR, and JIP-test) was added as a random effect to account for the repeated measures in QY_max modeling. Stepwise model simplification and selection were conducted based on Akaike Information Criteria (AIC) using bbmle package (Bolker and Bolker, 2020). Model fit was assessed by tests for overdispersion and residual fit tests using the DHARMa package (version 0.1.6) (Hartig and Hartig, 2017). Subsequently, the best model followed an analysis of variance (function Anova.glmmTMB) using the Wald $x^{2}$ statistic (Type II) function for statistical value. The curve-fitting procedure for analysis of rETR curve, IC, and OJIP curve was performed using ggplot2.

\subsection{Results}

\subsubsection{PSII maximum efficiency}

Prolonged photoperiod did not enhance PSII maximum efficiency (ФPSII). Two-way ANOVAs revealed that ФPSII was not significantly different between treatments $\left(X^{2}=0.54, p=0.464\right)$, but significantly different between ploidies $\left(X^{2}=80.08, p<0.000\right)$ (Table 3.3.). The ФPSII values between treatments among three cytotypes of $R$. auricomus were presented in Figure 3.1. The highest mean value of ФPSII was observed in the diploid control, and in hexaploids clone 29 of both control and stress treatment $(0.72$ (mean) \pm 0.03 (SD)), and the lowest mean value was observed in hexaploid clone 35 of the control treatment $(0.62 \pm 0.07)$ (Table S3). We report the test statistic for ФPSII to factors ploidy levels, treatments, and their interaction in generalized linear mixed models Table S4. Pairwise comparisons among different ploidies and hexaploid clones exhibited that the ФPSII of hexaploid clone 35 was significantly lower compared to other groups except for tetraploid stress treatments (Table S5). 


\subsubsection{Maximum quantum efficiency of PSII photochemistry}

The prolonged photoperiod did not enhance the maximum quantum efficiency of PSII photochemistry (QY_max). Two-way ANOVAs revealed that QY_max was not significantly different between treatments $\left(X^{2}=2.39, p=0.121\right)$, but significantly different between ploidies $\left(X^{2}=180.19, p<0.001\right)$ (Table 3.3.). The QY_max values between treatments among three cytotypes of $R$. auricomus were presented in Figure 3.1b. The highest mean value of QY_max was observed in tetraploids $(0.83 \pm 0.02)$ and hexaploid clone $29(0.83 \pm 0.01)$, both growing under control treatment. A slightly lower mean value of 0.81-0.82 was observed in diploids from both treatments and in tetraploids, and in hexaploid clone 29 growing under stress treatment. The hexaploid clone 35 had the lowest mean value of QY_max, i.e., $0.71 \pm 0.08$ in the control treatment and $0.66 \pm 0.09$ in stress treatment. The test statistics for the linear models of QY_max to factors ploidy, treatments, and their interaction in generalized linear mixed models were displayed in Table S6. Pairwise comparisons among different ploidies and hexaploid clones exhibited that QY-max of hexaploid clone 35 was significantly lower compared to other groups (Table S7).

\subsubsection{Relative electron transport rate}

The relative electron transport rate (rETR) curves linearly increased following the increase of light intensity, measured as photosynthesis photon flux density PPFD (Figure 3.2a). The mean values of rETR in each light intensity and their proportion alternation between the treatments is reported in Table 3.1, and boxplots are shown in Figure 3.3. Under extended photoperiod, rETR values were higher in diploids at 10 PPFD and in tetraploids at 10, 20, and 50 PPFD, but no significant alternation appeared in hexaploids. At higher light intensities, no differences appeared between treatments (Table 3.1). Kruskal-Wallis-Tests revealed that rETR from all light intensities (10-500 PPFD) were significantly different among diploids, tetraploid, and the two hexaploid clones in both treatments except in 10 PPFD of the control treatment (Table 3.2). Pairwise comparisons presented the significant values in each light intensity (Table S8). In the control treatment, the rETR of hexaploid clone 35 was significantly lower compared to tetraploids at lower light intensities (50 PPFD). But at higher light intensities (300 - 500 PPFD), tetraploids were significantly lower compared to hexaploid clone 29. In stress treatment, the ETR at a lower light intensity of hexaploid clone 35 was significantly lower compared to diploids (10-50 PPFD) and tetraploids (10 - 100 PPFD), but the ETR at higher light intensities (500 PPFD) of tetraploids was significantly lower compared to diploids. 
Table 3.3. $P$-values of the two-way ANOVAs for determination of the effects of prolong photoperiod on PSII maximum efficiency (DPSII) and maximum quantum efficiency of PSII photochemistry (QY_max) between treatment and among different ploidies and hexaploid clones. Significant results are in bold. QY_max: maximum photochemical efficiency of PSII photochemistry; ФPSII: actual quantum yield of PSII

\begin{tabular}{|c|c|c|c|c|c|c|}
\hline & \multicolumn{3}{|l|}{ (ФPSII) } & \multicolumn{3}{|c|}{ (QY_max) } \\
\hline & Chisq & Df & $\operatorname{Pr}(>$ Chisq $)$ & Chisq & Df & $\operatorname{Pr}(>$ Chisq $)$ \\
\hline Treatment & 0.536 & 1 & 0.46400 & 2.397 & 1 & 0.12160 \\
\hline Ploidy & 80.082 & 3 & $<0.00000$ & 180.187 & 3 & $<0.000001$ \\
\hline Treatment:Ploidy & 22.542 & 3 & 0.00005 & 1.474 & 3 & 0.68820 \\
\hline
\end{tabular}
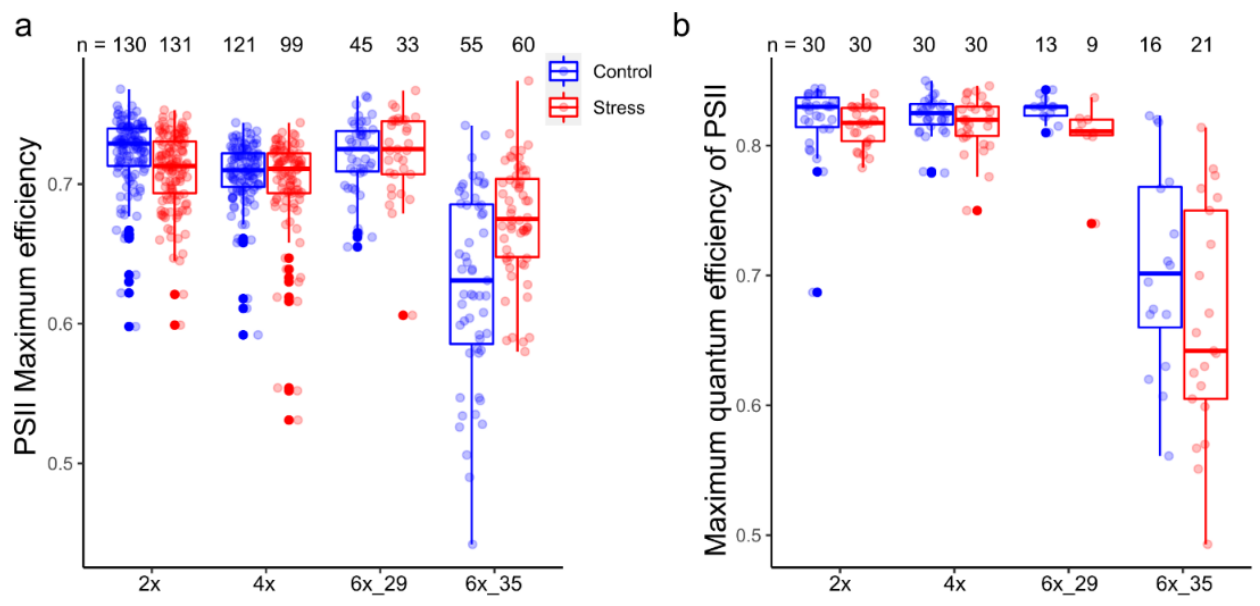

Figure 3.1 Photosynthesis performance of Ranunculus auricomus complex between treatment and among different ploidies and hexaploid clones. a. Boxplots of PSII maximum efficiency (ФPSII); b. Boxplots of maximum quantum efficiency of PSIl photochemistry (QY_max); Boxplots show the $25^{\text {th }}$, median, and $75^{\text {th }}$ percentile range, and jitter plots represent the exact data distribution. $\mathrm{n}=$ number of measurements from 3-14 individuals per group. 
a
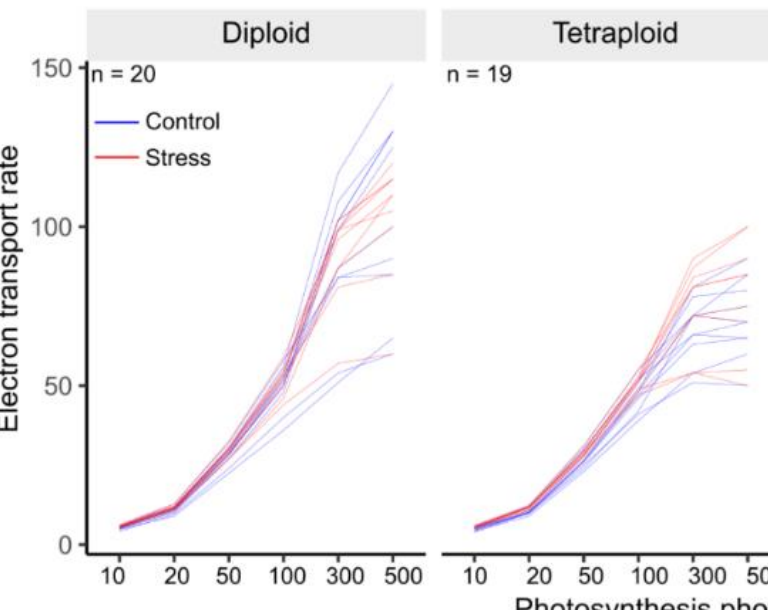

Hexaploid clone 29

Hexaploid clone 35

$n=8$ $n=10$

b

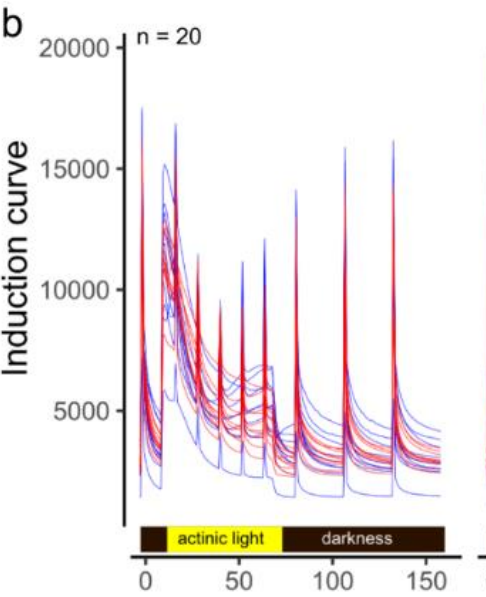

$n=20$
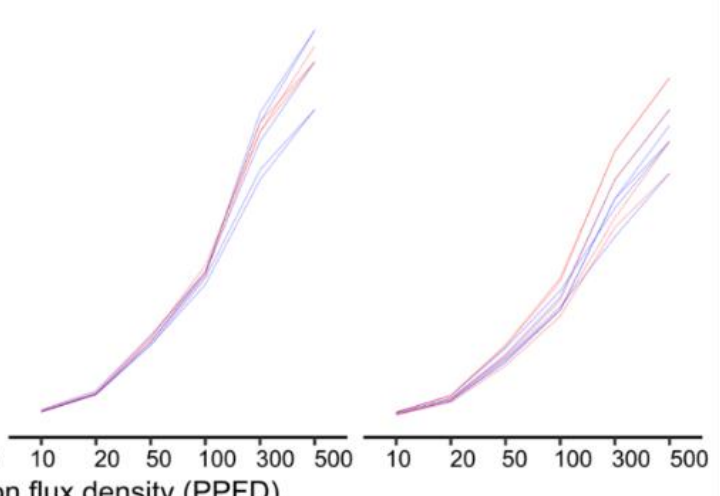
Photosynthesis photon flux density (PPFD)

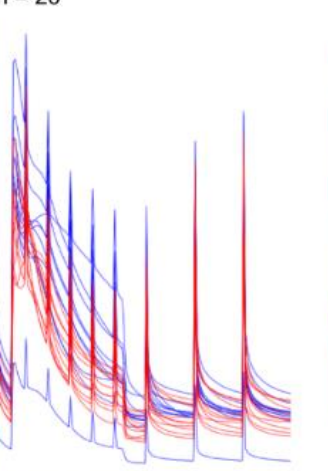

$n=7$

$n=13$

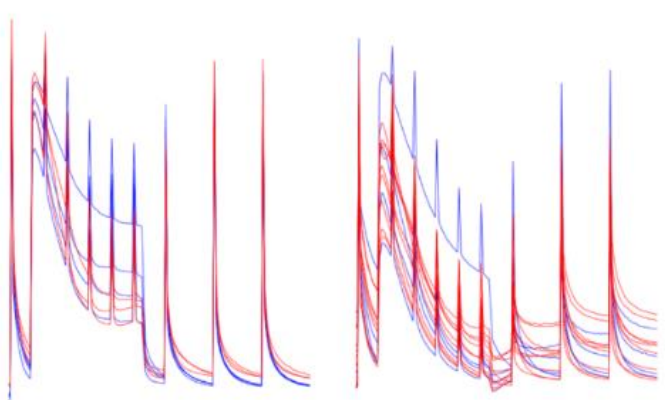

C
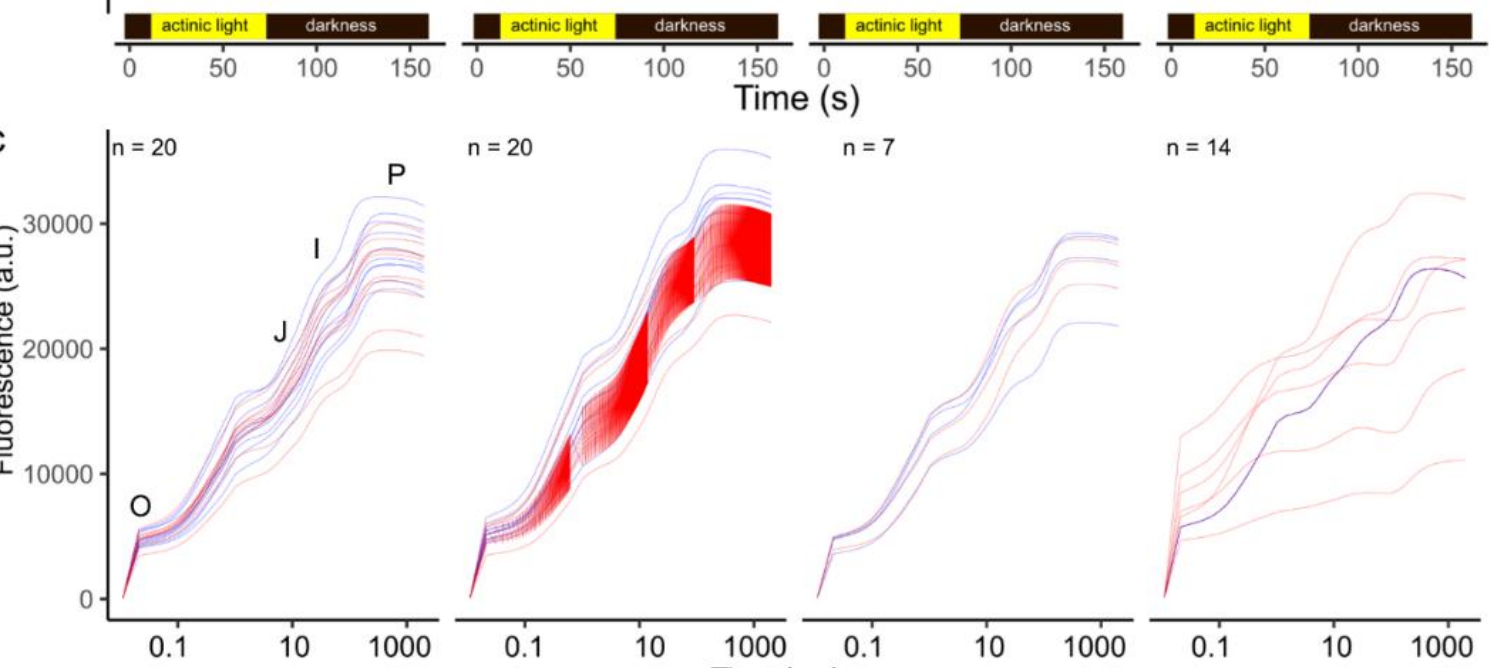
Time (s)

$n=20$

$\mathrm{n}=7$

$n=14$
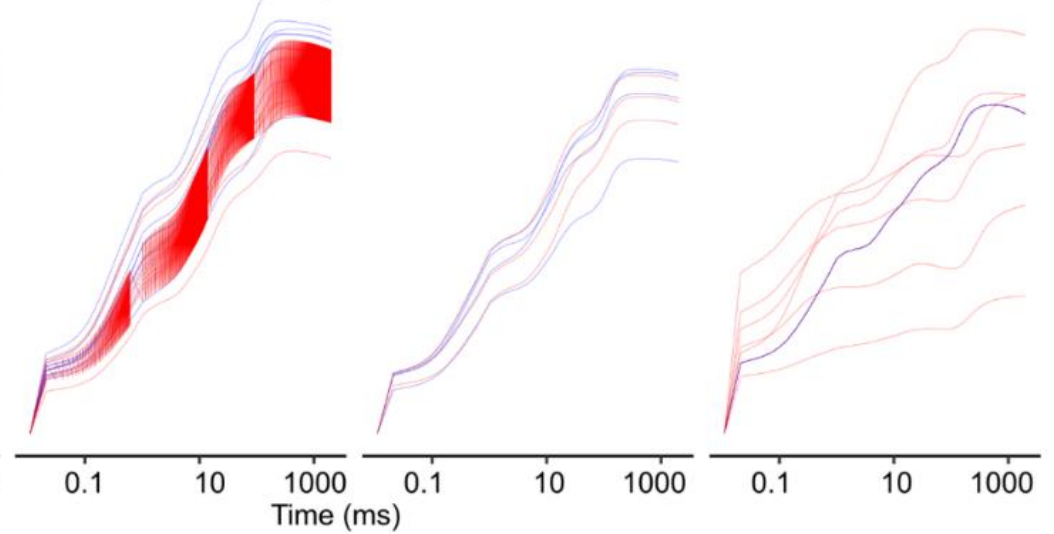

Figure 3.2 Effect photoperiod on the relative electron transport rate, induction curves, and fast fluorescence transient curves (OJIP) between treatment and among different ploidies and hexaploid clones. a. Relative electron transport rate ( $r E T R$ ) curve under different light intensities (10-500 PPFD). b. Induction and relaxation of non-photochemical quenching (NPQ) curves; c. OJIP curves. Each line in the curves is an individual value. $\mathrm{n}=$ number of measurements from 3-10 individuals per group. 

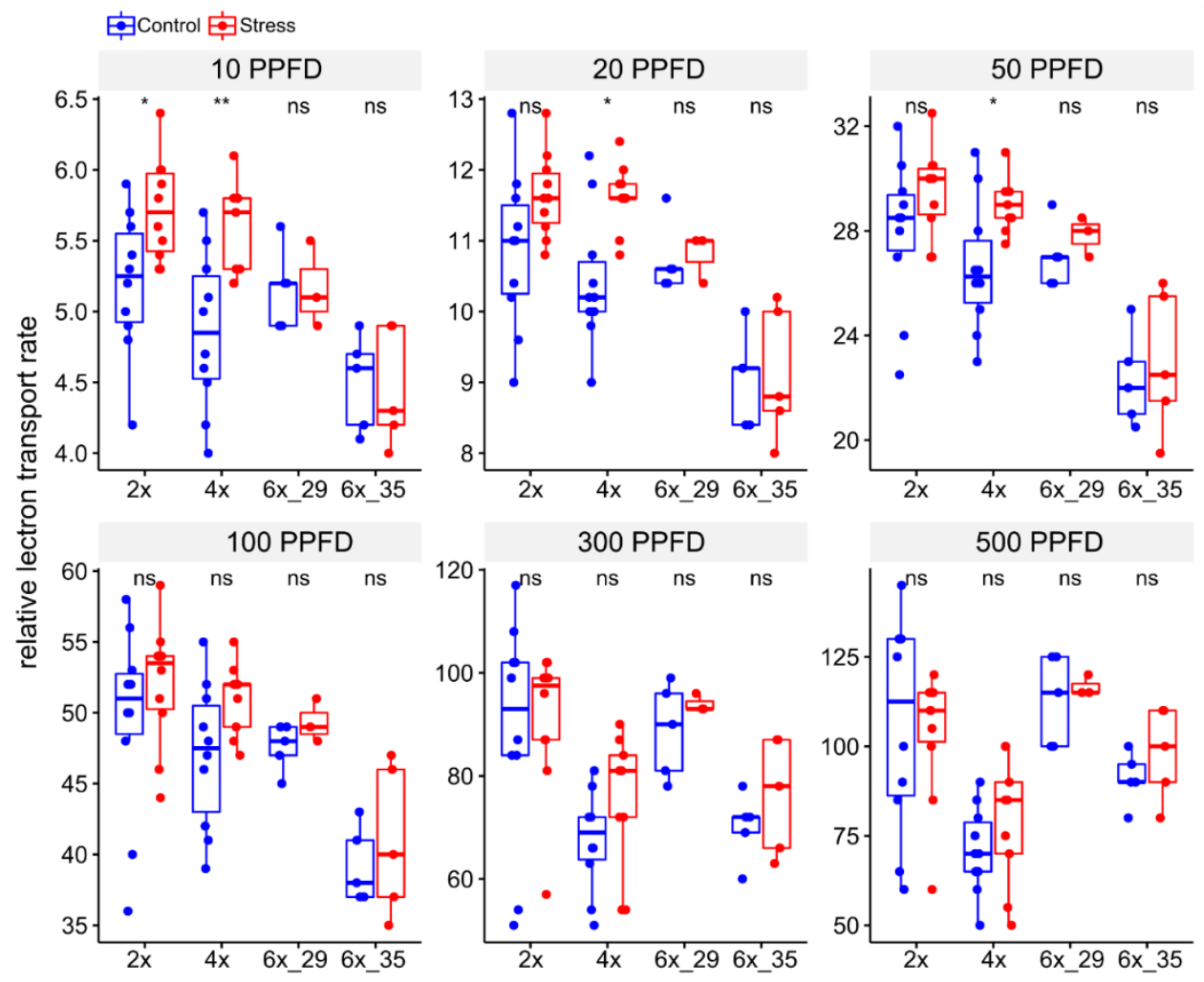

Figure 3.3 Photoperiod effect on relative electron transport rate between treatment and among different ploidies and hexaploid clones under different light intensities (10-500 PPFD). PPFD = photosynthesis photon flux density. Boxplots show the $25^{\text {th }}$, median, and $75^{\text {th }}$ percentile range, and jitter plots represent the exact data distribution. $n=3-10$ individuals per group, see details in Table 3.1.

\subsubsection{Fluorescence quenching analysis of induction curve}

The induction curves (IC) of all plants under actinic light and darkness were provided in Figure 3.2b. The IC was indicating a slightly higher fluorescence curve of plants under stress treatment even though a general overlapping pattern was observed. The quenching coefficients and other parameters extracted from the IC curve were provided in Table S9. The mean values of quenching parameters and their proportion alternation between the treatments were reported in Table 3.4 and visualized as boxplots in Figure 3.4. In diploids, extended light significantly increased four parameters, i.e., nonphotochemical quenching (NPQ) (S-C 20.44 $\%, p$-value $=0.0185)$, energy-dependent nonphotochemical quenching coefficient $\left(q_{E}\right)(S-C$ $23.60 \%$, p-value $=0.0147)$, fraction of NPQ related to $q_{E}\left(N P Q_{E}\right)(S-C 24.15 \%$, p-value $=$ 0.0232 ) and non-photochemical quenching coefficient ( $q M)(S-C 10.43 \%$, $p$-value $=0.0433$ ). In tetraploids, extended light significantly increased parameters of coefficient of photochemical quenching $(q P)(\mathrm{S}-\mathrm{C} 7.87 \%$, p-value $=0.0089)$ and non-photochemical that is formed only upon illumination $(q L)$ (S-C $15.04 \%$, p-value $=0.0147$ ). In the two hexaploid clones, no parameters were enhanced. Kruskal-Wallis tests of the IC parameters among different ploidies discriminated a total of eight parameters were altered only in control plants, i.e., NPQ, $q_{E}, q_{l}$, $N P Q_{E}, N P Q_{l}, q P$, and $q N$, while in stress treatment a total of five parameters. i.e., $P Q, q_{l}, N P Q_{l}$, 
$q N$, and $q L$ was altered (Table 3.2). Pairwise comparison marked the significant values among cytotype and hexaploid clones (Table S9). In control treatment, diploids performed a significantly lower NPQ (p.adj = 0.023), $q_{l}($ p.adj $=0.031)$, and $N P Q_{I}(p . a d j=0.031)$ than tetraploids, but diploids performed a significantly higher PQ than hexaploid clone 29 (p.adj = 0.012 ) and hexaploid clone 35 (p.adj = 0.048). Diploids also performed a significantly higher $q P$ than tetraploids (p.adj $=0.017$ ) and hexaploid clone 29 (p.adj $=0.012)$. Tetraploid performed a significantly higher $q_{I}(p . a d j=0.012)$ and $N P Q_{I}(p . a d j=0.012)$ than hexaploid clone 29. On the other hand, in stress treatment, hexaploid clone 35 performed a significantly lower $P Q$ than diploids ( .adj $=0.004$ ) and tetraploids ( $\mathrm{p} . \mathrm{adj}=0.002)$, but performed a significantly higher $q N$ than diploids (p.adj $=0.025$ ). Hexaploid clone 29 performed a significantly lower $q_{I}($ p.adj $=0.042)$ and $N P Q_{I}($ p.adj $=0.042)$ than tetraploids.

Table 3.1 Summary statistics and P- values of Wilcoxon-Mann-Whitney-Test for determination effects of prolonged photoperiod on relative electron transport rate between treatments under different light intensities (PPFD 10-500). S-C: proportion of alternation of the mean value, calculated as (Stress Control/(Stress + Control) ${ }^{*} 100$. Significant results are in bold

\begin{tabular}{|c|c|c|c|c|c|c|c|c|c|}
\hline \multirow{2}{*}{$\begin{array}{l}\text { Light } \\
\text { intensity }\end{array}$} & \multirow{2}{*}{ Ploidy } & \multicolumn{3}{|c|}{ Control treatment $(\mathrm{C})$} & \multicolumn{3}{|c|}{ Stress treatment $(\mathrm{S})$} & \multirow{2}{*}{ S - C (\%) } & \multirow{2}{*}{$p$-value } \\
\hline & & $\mathrm{n}$ & mean & sd & $\mathrm{n}$ & mean & sd & & \\
\hline \multirow[t]{4}{*}{10} & $2 x$ & 10 & 5.20 & 0.50 & 10 & 5.72 & 0.36 & 4.76 & 0.02070 \\
\hline & $4 x$ & 10 & 4.86 & 0.56 & 9 & 5.58 & 0.33 & 6.88 & 0.00601 \\
\hline & $6 x \_29$ & 5 & 5.16 & 0.29 & 3 & 5.17 & 0.31 & 0.07 & 0.87800 \\
\hline & $6 \times 35$ & 5 & 4.50 & 0.34 & 5 & 4.46 & 0.42 & -0.45 & 1.00000 \\
\hline \multirow[t]{4}{*}{20} & $2 x$ & 10 & 10.86 & 1.11 & 10 & 11.64 & 0.60 & 3.47 & 0.07450 \\
\hline & $4 x$ & 10 & 10.44 & 0.95 & 9 & 11.62 & 0.48 & 5.36 & 0.01230 \\
\hline & $6 x \_29$ & 5 & 10.72 & 0.50 & 3 & 10.80 & 0.35 & 0.37 & 0.64300 \\
\hline & $6 \times 35$ & 5 & 9.04 & 0.67 & 5 & 9.12 & 0.94 & 0.44 & 0.83300 \\
\hline \multirow[t]{4}{*}{50} & $2 x$ & 10 & 27.95 & 2.85 & 10 & 29.50 & 1.68 & 2.70 & 0.18300 \\
\hline & $4 x$ & 10 & 26.60 & 2.49 & 9 & 29.00 & 1.03 & 4.32 & 0.02690 \\
\hline & $6 x \_29$ & 5 & 27.00 & 1.23 & 3 & 27.83 & 0.76 & 1.52 & 0.28200 \\
\hline & $6 x \_35$ & 5 & 22.30 & 1.79 & 5 & 23.00 & 2.74 & 1.55 & 0.60200 \\
\hline \multirow[t]{4}{*}{100} & $2 x$ & 10 & 49.50 & 6.79 & 10 & 52.00 & 4.42 & 2.46 & 0.34300 \\
\hline & $4 x$ & 10 & 47.00 & 5.12 & 9 & 51.00 & 2.55 & 4.08 & 0.05870 \\
\hline & $6 x \_29$ & 5 & 47.60 & 1.67 & 3 & 49.33 & 1.53 & 1.79 & 0.21900 \\
\hline & $6 x+35$ & 5 & 39.20 & 2.68 & 5 & 41.00 & 5.34 & 2.24 & 0.75100 \\
\hline \multirow[t]{4}{*}{300} & $2 x$ & 10 & 88.80 & 21.87 & 10 & 90.90 & 13.93 & 1.17 & 0.90900 \\
\hline & $4 x$ & 10 & 67.50 & 9.62 & 9 & 75.00 & 13.33 & 5.26 & 0.09810 \\
\hline & $6 x \_29$ & 5 & 88.80 & 9.15 & 3 & 94.00 & 1.73 & 2.85 & 0.54600 \\
\hline & $6 x \_35$ & 5 & 70.20 & 6.57 & 5 & 76.20 & 11.35 & 4.10 & 0.39900 \\
\hline \multirow[t]{4}{*}{500} & $2 x$ & 10 & 106.00 & 30.07 & 10 & 103.50 & 18.27 & -1.19 & 0.56900 \\
\hline & $4 x$ & 10 & 71.00 & 11.97 & 9 & 78.89 & 17.99 & 5.26 & 0.23400 \\
\hline & $6 x \_29$ & 5 & 113.00 & 12.55 & 3 & 116.67 & 2.89 & 1.60 & 0.87700 \\
\hline & $6 x \_35$ & 5 & 91.00 & 7.42 & 5 & 98.00 & 13.04 & 3.70 & 0.33700 \\
\hline
\end{tabular}


Table $3.2 P$-values of Kruskal-Wallis-Tests for determination of significant differences of relative electron transport rate, induction curve parameters, and specific energy fluxes parameters of JIP-test among different ploidies and hexaploid clones. Significant results are in bold

\begin{tabular}{|c|c|c|c|c|c|c|c|c|}
\hline & \multicolumn{4}{|c|}{ Control treatment } & \multicolumn{4}{|c|}{ Stress treatment } \\
\hline & $\mathbf{n}$ & statistic & df & p-value & $\mathbf{n}$ & statistic & df & p-value \\
\hline \multicolumn{9}{|c|}{ Electron transport rate at PPFD } \\
\hline 10 & 30 & 7.61 & 3 & 0.0547 & 27 & 14.97 & 3 & 0.00184 \\
\hline 20 & 30 & 10.26 & 3 & 0.0165 & 27 & 15.06 & 3 & 0.00177 \\
\hline 50 & 30 & 11.49 & 3 & 0.00934 & 27 & 14.42 & 3 & 0.00239 \\
\hline 100 & 30 & 9.61 & 3 & 0.0222 & 27 & 11.53 & 3 & 0.00919 \\
\hline 300 & 30 & 11.72 & 3 & 0.00839 & 27 & 11.06 & 3 & 0.0114 \\
\hline 500 & 30 & 13.68 & 3 & 0.00337 & 27 & 13.32 & 3 & 0.00399 \\
\hline \multicolumn{9}{|c|}{ Inductive curve parameters } \\
\hline NPQ & 28 & 13.31 & 3 & 0.00402 & 32 & 6.88 & 3 & 0.07570 \\
\hline$P Q$ & 28 & 12.01 & 3 & 0.00736 & 32 & 17.14 & 3 & 0.00066 \\
\hline$q_{E}$ & 28 & 12.22 & 3 & 0.00668 & 32 & 6.50 & 3 & 0.08980 \\
\hline $\mathrm{q}_{l}$ & 28 & 13.16 & 3 & 0.00431 & 32 & 10.66 & 3 & 0.01370 \\
\hline$N P Q_{E}$ & 28 & 12.89 & 3 & 0.00489 & 32 & 7.65 & 3 & 0.05380 \\
\hline$N P Q_{1}$ & 28 & 13.16 & 3 & 0.00431 & 32 & 10.66 & 3 & 0.01370 \\
\hline $\mathrm{qP}$ & 28 & 12.08 & 3 & 0.00713 & 32 & 5.79 & 3 & 0.12200 \\
\hline $\mathrm{qN}$ & 28 & 11.97 & 3 & 0.00749 & 32 & 12.05 & 3 & 0.00723 \\
\hline $\mathrm{qL}$ & 28 & 7.26 & 3 & 0.06400 & 32 & 9.72 & 3 & 0.02110 \\
\hline \multicolumn{9}{|c|}{ Specific energy fluxes parameters of JIP-test } \\
\hline Pl_Abs & 31 & 14.69 & 3 & 0.0021 & 31 & 14.36 & 3 & 0.00246 \\
\hline $\mathrm{ABS} / \mathrm{RC}$ & 31 & 9.29 & 3 & 0.0256 & 31 & 13.79 & 3 & 0.00321 \\
\hline TRo/RC & 31 & 17.44 & 3 & 0.000573 & 31 & 16.80 & 3 & 0.000775 \\
\hline $\mathrm{ETo} / \mathrm{RC}$ & 31 & 1.76 & 3 & 0.624 & 31 & 16.36 & 3 & 0.000957 \\
\hline Dlo/RC & 31 & 12.62 & 3 & 0.00553 & 31 & 17.17 & 3 & 0.000651 \\
\hline
\end{tabular}



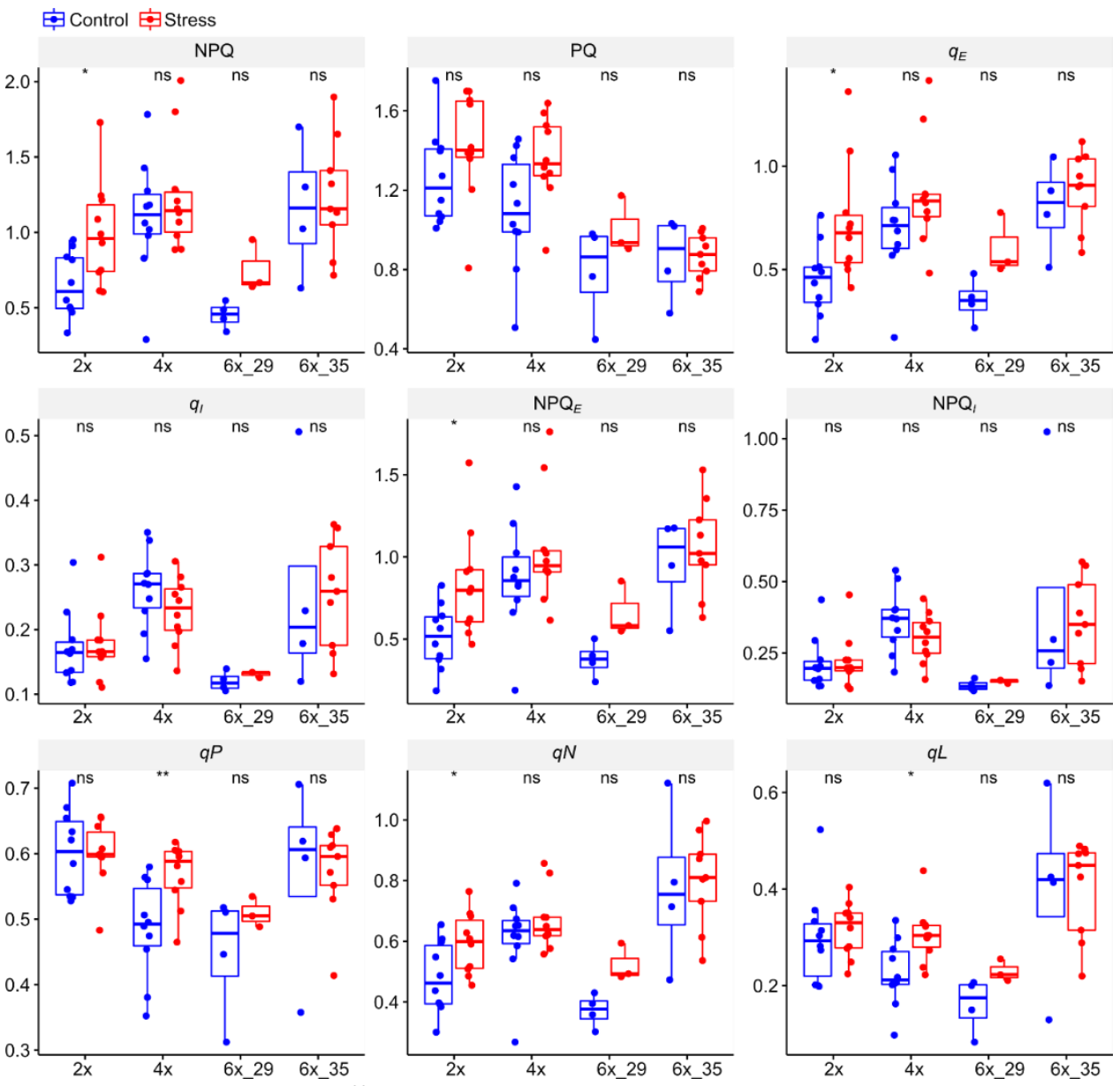

Figure 3.4 Photoperiod effect on induction curve parameters between treatment and among different ploidies and hexaploid clones and hexaploid clones. $\mathrm{NPQ}=$ Non-photochemical quenching, $\mathrm{NPQ}_{E}=$ energy dependent non-photochemical $\mathrm{Chl}$ fluorescence quenching, $\mathrm{NPQ} /=$ photoinhibitory nonphotochemical $\mathrm{Chl}$ fluorescence quenching, $\mathrm{PQ}=$ photochemical quenching, $q_{E}=$ energy dependent non-photochemical $\mathrm{Chl}$ fluorescence quenching coefficient, $q_{l}=$ photoinhibitory non-photochemical $\mathrm{Chl}$ fluorescence quenching coefficient, $q P=$ quenching coefficient of photochemical, $q N$ = quenching coefficient of non-photochemical, $q L$ = quenching coefficient of non-photochemical that is formed only upon illumination to regulate the amount of absorbed light in avoiding damage effect. Boxplots show the $25^{\text {th }}$, median, and $75^{\text {th }}$ percentile range, and jitter plots represent the exact data distribution. $n=3$ 10 individuals per group, see detail in Table 3.3. 
Table 3.3 Summary statistic and $P$ - values of Wilcoxon-Mann-Whitney-Test for determination of effects of prolonged photoperiod on induction curves parameters between treatments . S-C: proportion of alternation of the mean value, calculated as (Stress - Control/(Stress + Control) ${ }^{\star} 100$. Significant results are in bold.

\begin{tabular}{|c|c|c|c|c|c|c|c|c|c|}
\hline \multirow{2}{*}{ Parameter } & \multirow{2}{*}{ Ploidy } & \multicolumn{3}{|c|}{ Control treatment $(\mathrm{C})$} & \multicolumn{3}{|c|}{ Stress treatment (S) } & \multirow{2}{*}{ S - C (\%) } & \multirow{2}{*}{ p-value } \\
\hline & & $\mathbf{n}$ & mean & sd & $\mathbf{n}$ & mean & sd & & \\
\hline \multirow[t]{4}{*}{$N P Q$} & $2 x$ & 10 & 0.654 & 0.213 & 10 & 0.99 & 0.347 & 20.44 & 0.0185 \\
\hline & $4 \mathrm{x}$ & 10 & 1.102 & 0.39 & 10 & 1.241 & 0.376 & 5.93 & 0.631 \\
\hline & $6 x \_29$ & 4 & 0.451 & 0.088 & 3 & 0.753 & 0.173 & 25.08 & 0.0571 \\
\hline & $6 x \_35$ & 4 & 1.164 & 0.451 & 9 & 1.237 & 0.381 & 3.04 & 0.71 \\
\hline \multirow[t]{4}{*}{$P Q$} & $2 x$ & 10 & 1.263 & 0.238 & 10 & 1.425 & 0.276 & 6.03 & 0.218 \\
\hline & $4 \mathrm{x}$ & 10 & 1.093 & 0.296 & 10 & 1.358 & 0.218 & 10.81 & 0.0524 \\
\hline & $6 x \_29$ & 4 & 0.789 & 0.248 & 3 & 1.004 & 0.147 & 11.99 & 0.629 \\
\hline & $6 \times 35$ & 4 & 0.856 & 0.214 & 9 & 0.869 & 0.111 & 0.75 & 0.71 \\
\hline \multirow[t]{4}{*}{$q_{E}$} & $2 x$ & 10 & 0.45 & 0.178 & 10 & 0.728 & 0.289 & 23.60 & 0.0147 \\
\hline & $4 x$ & 10 & 0.698 & 0.244 & 10 & 0.869 & 0.269 & 10.91 & 0.123 \\
\hline & $6 x \_29$ & 4 & 0.35 & 0.108 & 3 & 0.605 & 0.149 & 26.70 & 0.0571 \\
\hline & $6 x \_35$ & 4 & 0.801 & 0.225 & 9 & 0.889 & 0.18 & 5.21 & 0.414 \\
\hline \multirow[t]{4}{*}{$q_{1}$} & $2 x$ & 10 & 0.172 & 0.057 & 10 & 0.178 & 0.057 & 1.71 & 0.912 \\
\hline & $4 x$ & 10 & 0.263 & 0.06 & 10 & 0.229 & 0.052 & -6.91 & 0.19 \\
\hline & $6 x \_29$ & 4 & 0.12 & 0.015 & 3 & 0.131 & 0.005 & 4.38 & 0.4 \\
\hline & $6 x \_35$ & 4 & 0.258 & 0.171 & 9 & 0.256 & 0.085 & -0.39 & 0.71 \\
\hline \multirow[t]{4}{*}{$N P Q_{E}$} & $2 x$ & 10 & 0.512 & 0.198 & 10 & 0.838 & 0.33 & 24.15 & 0.0232 \\
\hline & $4 x$ & 10 & 0.871 & 0.33 & 10 & 1.044 & 0.349 & 9.03 & 0.315 \\
\hline & $6 x \_29$ & 4 & 0.375 & 0.109 & 3 & 0.662 & 0.167 & 27.68 & 0.0571 \\
\hline & $6 x \_35$ & 4 & 0.962 & 0.294 & 9 & 1.06 & 0.289 & 4.85 & 0.604 \\
\hline \multirow[t]{4}{*}{$N P Q_{1}$} & $2 x$ & 10 & 0.213 & 0.092 & 10 & 0.222 & 0.093 & 2.07 & 0.912 \\
\hline & $4 x$ & 10 & 0.365 & 0.11 & 10 & 0.302 & 0.086 & -9.45 & 0.19 \\
\hline & $6 x \_29$ & 4 & 0.136 & 0.02 & 3 & 0.151 & 0.006 & 5.23 & 0.4 \\
\hline & $6 x \_35$ & 4 & 0.419 & 0.409 & 9 & 0.359 & 0.156 & -7.71 & 0.71 \\
\hline \multirow[t]{4}{*}{$q P$} & $2 x$ & 10 & 0.601 & 0.065 & 10 & 0.6 & 0.05 & -0.08 & 0.912 \\
\hline & $4 x$ & 10 & 0.486 & 0.075 & 10 & 0.569 & 0.049 & 7.87 & 0.00893 \\
\hline & $6 x \_29$ & 4 & 0.447 & 0.095 & 3 & 0.509 & 0.023 & 6.49 & 0.629 \\
\hline & $6 x \_35$ & 4 & 0.569 & 0.149 & 9 & 0.573 & 0.069 & 0.35 & 0.825 \\
\hline \multirow[t]{4}{*}{$q N$} & $2 x$ & 10 & 0.481 & 0.117 & 10 & 0.593 & 0.101 & 10.43 & 0.0433 \\
\hline & $4 x$ & 10 & 0.611 & 0.139 & 10 & 0.669 & 0.099 & 4.53 & 0.529 \\
\hline & $6 x \_29$ & 4 & 0.371 & 0.055 & 3 & 0.524 & 0.061 & 17.09 & 0.0571 \\
\hline & $6 x \_35$ & 4 & 0.776 & 0.268 & 9 & 0.802 & 0.153 & 1.65 & 0.604 \\
\hline \multirow[t]{4}{*}{$q L$} & $2 x$ & 10 & 0.299 & 0.097 & 10 & 0.317 & 0.057 & 2.92 & 0.353 \\
\hline & $4 x$ & 10 & 0.226 & 0.069 & 10 & 0.306 & 0.059 & 15.04 & 0.0147 \\
\hline & $6 x \_29$ & 4 & 0.16 & 0.057 & 3 & 0.23 & 0.023 & 17.95 & 0.0571 \\
\hline & 6x_35 & 4 & 0.397 & 0.202 & 9 & 0.402 & 0.101 & 0.63 & 0.71 \\
\hline
\end{tabular}




\subsubsection{Fast fluorescence transient curve}

All fast fluorescence transient curve (OJIP) transients among ploidies from both light treatments showed a typical polyphasic rise of the OJIP curve with a slight decrease in stressed diploids at the I-P phase, tetraploids at the $\mathrm{J}-\mathrm{I}$ and I-P phase, and a higher alternation of all phase in hexaploid clone 35. The hexaploid clone 29 did not show a clear shift of the OJIP phase between treatments (Figure 3.2c). The JIP-test parameters were provided in Table S10. The mean values of the JIP-test and their proportion alternation between the treatments were reported in Table 3.4 and visualized as boxplots in Figure 3.5. The JIP-test revealed that extended light significantly altered a total of fourteen parameters in diploids and a total of two parameters in tetraploids and hexaploid clone 35 , but none of the parameters were altered in hexaploid clone 29. Stress in diploids altered the proportion of alternation of the mean S-C value by decreasing $\mathrm{F}_{\mathrm{M}} / \mathrm{F}_{0}(-4.40 \%), \mathrm{F}_{\mathrm{V}} / \mathrm{F}_{0}(-5.38 \%), \mathrm{Fv}_{\mathrm{V}} / \mathrm{F}_{\mathrm{M}}$ or Phi_P $(-0.98$ $\%)$, Phi_E $E_{0}(-3.25 \%), S_{s}(-3.96 \%)$, and Pi_Abs (-15.66\%), by increasing: $\mathrm{M}_{0}(7.25 \%)$, Vi (3.31 $\%)$, Phi_Pav (0.51\%), Phi_D 0 (4.35\%), ABS/RC (4.71\%), TR/RC (3.75\%), and DI $/$ RC (9.07 $\%)$. Stress in tetraploids decreased the S-C parameter for Fix.Area (-14.12\%) and ET/RC ($5.09 \%)$. Stress in the hexaploid clone 35 increased the S-C parameter for TRo/RC $(8.95 \%)$ and $\mathrm{ET}_{\mathrm{o}} / \mathrm{RC}$ (13.72\%) (see p-values in Table S10). Selected parameters, namely the specific energy fluxes and PI_Abs, were presented in Table 3.4 and visualized as boxplots in Figure 3.5. Kruskal-Wallis tests of the specific energy fluxes and PI_Abs parameters among different ploidy indicated that all parameters were significantly different in both treatments except $\mathrm{ET}_{0} / \mathrm{RC}$ in the control treatment (Table 3.2). Pairwise comparison marked the significant values between cytotypes (Table S12). In both treatments, hexaploid clone 35 had a lower mean value of PI_Abs compared to diploids and tetraploids, but had a higher mean value of $\mathrm{ABS} / \mathrm{RC}$ and $\mathrm{DI} / \mathrm{RC}$ compared to diploids and tetraploid, and a higher mean value of $\mathrm{TR}_{0} / \mathrm{RC}$ than diploids, tetraploids, and hexaploid clone 29 (only control treatment). In the stress treatment, tetraploids had a lower mean value of $\mathrm{ET}_{0} / \mathrm{RC}$ than diploids and hexaploid clone 35.

\subsection{Discussion}

Plant growth and development are sensitive to the photoperiod, which affects the metabolite profile (de Castro et al., 2019), photosynthesis (Bauerle et al., 2012; Sulpice et al., 2014; Kinoshita et al., 2020), growth (Wu et al., 2004; Fortini et al., 2020), and flower initiation (Jeong and Clark, 2005). Extended photoperiod altered the mode of reproduction in hexaploid plants of the Ranunculus auricomus complex by increasing proportions of sexual ovules (Klatt et al., 2016) and showed a more substantial effect in lower ploidy levels (Ulum et al., 2020). The present study analyzed the photosynthesis efficiency of three cytotypes of Ranunculus 
auricomus under control and stress conditions with extended photoperiod. Combining the current result with our previous study on the same plant material (Ulum et al., 2020), we tested the hypothesis that extended photoperiod alters the photosynthetic performance, with the expectation of a buffering effect by polyploidy.
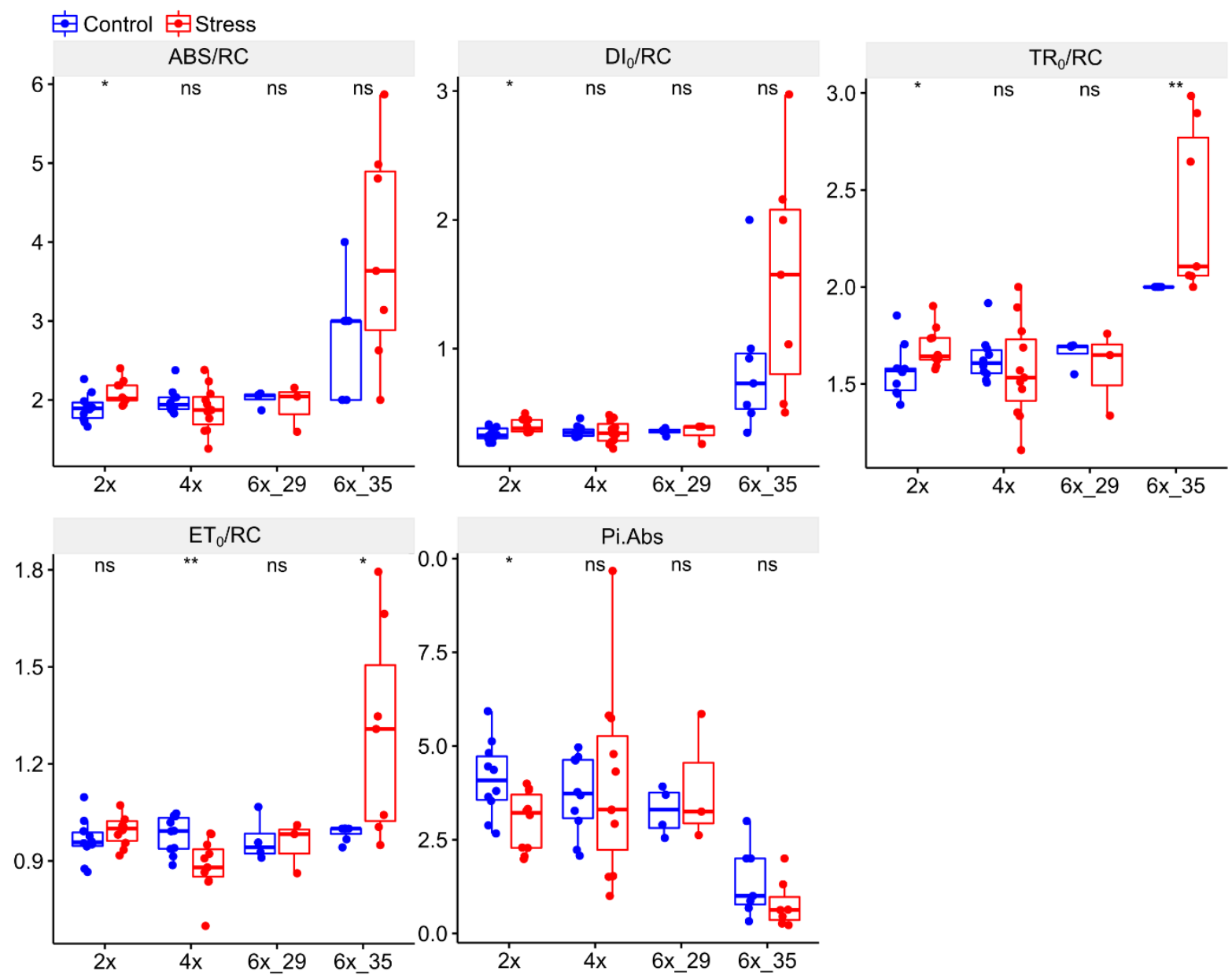

Figure 3.5 Photoperiod effect on specific energy fluxes parameters of JIP-test between treatment and among different ploidies and hexaploid clones. $A B S / R C=$ absorption flux per reaction center (RC) (apparent antenna size of an active PSII), $D I_{0} / R C=$ dissipated energy flux per reaction center $(\mathrm{RC}), \mathrm{ET} / \mathrm{RC}=$ electron transport flux per reaction center $(\mathrm{RC}), \mathrm{Pi}$ Abs = performance index on absorption basis related to the overall photosynthetic activity of PSII, TRo/RC = trapped energy flux per RC. Boxplots show the $25^{\text {th }}$, median, and $75^{\text {th }}$ percentile range, and jitter plots represent the exact data distribution. $n=3-11$ individuals per group, see detail in Table 3.4

\subsubsection{Ploidy dependent effects of photoperiod on photosynthetic capacity}

Photoperiod alternation modulating plant photosynthesis was reported in several studies (e.g., (Fréchette et al., 2016; de Castro et al., 2019; Elkins and van lersel, 2020). Here we examined the prolonged photoperiod effects on photosynthesis performance of $R$. auricomus cytotypes marked by ФPSII, rETR, and QY_max. The three cytotypes of $R$. auricomus complex exhibited in either short $(10 \mathrm{~h})$ or long $(16.5 \mathrm{~h})$ photoperiod a similar capacity to capture the climatic chamber's light for the PSII (photosystem II) photochemistry under different photoperiod 
treatments, as observed in our ФPSII data. The ФPSII photosynthesis parameter estimates the theoretical proportion of absorbed light used by Chlorophyll associated with PSII and hence measures the efficiency of photosystem II (Ghashghaie et al., 1992; Baker, 2008). However, there was a significant difference between ploidy levels (Table 3.3.), caused mainly by lower $\Phi$ PSII values of the hexaploid clone 35.

The light intensity of our climatic chamber $\left(250 \mathrm{~m}^{-2} \mathrm{~s}^{-1}\right)$ provided sufficient energy for the reaction center in PSII and kept the PSII reaction center at the open stage as indicated by relative electron transport (rETR) values at 100 and 300 PPFD (Figure 3.3). rETR curve illustrates the physiological flexibility of plant photosynthetic capacity to rapid irradiation changes (Schreiber and Berry, 1977; Ralph and Gademann, 2005). In our study, rETR confirmed that in lower light intensities (10-50 PPFD), diploids and tetraploids had a higher photosynthetic capacity after longer photoperiods treatment, but no differences appeared at higher light intensities (100-500 PPFD) in both treatments. The increase of rETR in diploids and tetraploids might be related to the convexity response of DPSII to Photosynthetic photon flux density (PPFD) as the same pattern was reported in Lactuca sativa (Weaver and van lersel, 2020). Our rETR curves also suggested different photosynthesis performances between ploidy and hexaploid clones (Table S8). Diploids and hexaploid clone 29 were more adaptive to a wider range of light intensity, but tetraploids and hexaploid clone 35 were more sensitive to the change of light intensity. Tetraploids performed a better rETR in lower light intensity (<300 PPFD), but hexaploid clone 35 improved the rETR within the increase of light intensity (>300 PPFD). The rETR values, however, must be interpreted with caution as they are calculated on assumptions of constant values for a fraction of absorbed PPFD that is received by PSII (0.5), and these assumptions are often not met (Baker, 2008).

Plants under excess light exposure might damage photosystem II or activate photoinhibition in the photosynthetic reaction center (RC) of PSII (Takahashi and Murata, 2008). $\mathrm{C}_{3}$ plants have relatively constant $F_{v} / F_{m}$ ratios, with mean values of $Q Y$ max of $\sim 0.83$ under unstressed conditions, whereas deviating values indicate the presence of photoinhibition (Björkman and Demmig, 1987). The photoperiod treatments did not activate photoinhibition in $R$. auricomus as indicated by the maximum quantum efficiency of PSII photochemistry (QY_max) in almost all plants around 0.83 , except for the hexaploid clone 35 . In this clone, the low QY_max value might be related to photoinhibition, as indicated by ФPSII and rETR. However, this clone also exhibited a slower development of leaves during the measurement period, which also may explain deviating results. The QY_max value of 0.81 from the congeneric species Ranunculus asiaticus L. was reported to be close to the optimal value (Carillo et al., 2019). The result of our study with a range of mean values of QY_max between $0.81-0.83$ in almost all of the 
plants was close to the optimal value, suggesting that in the presence of light excess $R$. auricomus is prone to perform heat dissipation as the main photoprotective mechanism. Another study in Lippia alba also confirmed the same pattern that photoperiod extension affected the NPQ and ETR, but neither affected the QY_max (de Castro et al., 2019).

Table 3.4 Summary statistics and $P$ - values of Wilcoxon-Mann-Whitney-Test for determination of effects of prolonged photoperiod on specific energy fluxes parameters of JIP-test between treatments . S-C: proportion of alternation of the mean value, calculated as (Stress - Control/(Stress + Control) ${ }^{*} 100$. Significant results are in bold

\begin{tabular}{|c|c|c|c|c|c|c|c|c|c|}
\hline \multirow{2}{*}{ Parameter } & \multirow{2}{*}{ Ploidy } & \multicolumn{3}{|c|}{ Control treatment $(\mathrm{C})$} & \multicolumn{3}{|c|}{ Stress treatment $(\mathrm{S})$} & \multirow[t]{2}{*}{ S - C (\%) } & \multirow[t]{2}{*}{$p$-value } \\
\hline & & $\mathrm{n}$ & mean & sd & $\mathrm{n}$ & mean & sd & & \\
\hline \multirow[t]{4}{*}{$\mathrm{ABS} / \mathrm{RC}$} & $2 x$ & 10 & 1.902 & 0.181 & 10 & 2.09 & 0.154 & 4.709 & 0.01400 \\
\hline & $4 x$ & 10 & 1.987 & 0.162 & 11 & 1.885 & 0.29 & -2.634 & 0.38700 \\
\hline & $6 x \_29$ & 4 & 2.017 & 0.099 & 3 & 1.931 & 0.295 & -2.178 & 0.85700 \\
\hline & $6 x \_35$ & 7 & 2.714 & 0.756 & 7 & 3.866 & 1.399 & 17.508 & 0.10500 \\
\hline \multirow[t]{4}{*}{$\mathrm{DI} / \mathrm{RC}$} & $2 x$ & 10 & 0.336 & 0.052 & 10 & 0.403 & 0.054 & 9.066 & 0.01150 \\
\hline & $4 \mathrm{x}$ & 10 & 0.356 & 0.045 & 11 & 0.354 & 0.087 & -0.282 & 0.86300 \\
\hline & $6 x \_29$ & 4 & 0.357 & 0.028 & 3 & 0.35 & 0.078 & -0.990 & 0.62900 \\
\hline & $6 x \_35$ & 7 & 0.867 & 0.551 & 7 & 1.545 & 0.905 & 28.109 & 0.12800 \\
\hline \multirow[t]{4}{*}{$\mathrm{TR} / \mathrm{RC}$} & $2 x$ & 10 & 1.566 & 0.134 & 10 & 1.688 & 0.102 & 3.749 & 0.01470 \\
\hline & $4 \mathrm{x}$ & 10 & 1.631 & 0.12 & 11 & 1.572 & 0.251 & -1.842 & 0.42600 \\
\hline & $6 x \_29$ & 4 & 1.659 & 0.073 & 3 & 1.582 & 0.219 & -2.376 & 0.85700 \\
\hline & $6 \times 35$ & 7 & 2 & 0 & 7 & 2.393 & 0.434 & 8.946 & 0.00372 \\
\hline \multirow[t]{4}{*}{$\mathrm{ET}_{0} / \mathrm{RC}$} & $2 x$ & 10 & 0.964 & 0.067 & 10 & 0.992 & 0.047 & 1.431 & 0.21200 \\
\hline & $4 x$ & 10 & 0.981 & 0.057 & 11 & 0.886 & 0.081 & -5.088 & 0.00365 \\
\hline & $6 x \_29$ & 4 & 0.965 & 0.071 & 3 & 0.952 & 0.079 & -0.678 & 1.00000 \\
\hline & $6 x \_35$ & 7 & 0.987 & 0.023 & 7 & 1.301 & 0.331 & 13.724 & 0.01870 \\
\hline \multirow[t]{4}{*}{ Pi_Abs } & $2 x$ & 10 & 4.121 & 1.014 & 10 & 3.005 & 0.786 & -15.661 & 0.02880 \\
\hline & $4 x$ & 10 & 3.696 & 1.044 & 11 & 3.99 & 2.502 & 3.825 & 1.00000 \\
\hline & $6 x \_29$ & 4 & 3.267 & 0.65 & 3 & 3.909 & 1.715 & 8.946 & 0.85700 \\
\hline & $6 \times 35$ & 7 & 1.41 & 0.949 & 7 & 0.787 & 0.645 & -28.357 & 0.12400 \\
\hline
\end{tabular}

\subsubsection{Ploidy dependent effects of photoperiod on quenching processes}

The photosynthesis pigments in plants absorb the energy from the photon, which is mostly used in photosynthetic electron transport (photochemical quenching), but the excess light energy is dissipated as heat (non-photochemical quenching) and/or re-emitted as fluorescence (Walker, 1987). These three fates occur in competition, as they rely on the same energy source, and the increase of one factor decreases the others (Kautsky and Hirsch, 1931; Maxwell and Johnson, 2000). Heat dissipation was observed to some extent in both photoperiods in this study. An enhancement of the non-photochemical quenching after extended photoperiod was observed only in diploids but absent in tetraploids and hexaploids, indicating the stress buffer in polyploid plants. NPQ corresponds to the excess light dissipation 
to heat (Brestic and Zivcak, 2013). A better capacity of polyploids for regulating nonphotochemical quenching was also observed in tetraploid Glycine max (Coate et al., 2012). A higher photosynthesis rate and better quenching of destructive light in polyploids compared to their diploid progenitor were suggested as the advantages of the higher DNA content per cell (Warner and Edwards, 1993) and more photosynthetic pigments as reported in polyploids of Vicia cracca (Münzbergová and Haisel, 2019).

The component of NPQ is discriminated into $q_{E}$ quenching and $q_{1}$ quenching based on relaxation analysis in high illumination (D’Ambrosio et al., 2008; Lazár, 2015). In diploids, prolonged photoperiod activated mechanism of thermal dissipation as indicated by $\mathrm{NPQ}_{E}$ and $q_{E}$, instead of photoinhibition of photosynthesis (NPQ, and $q_{l}$ ). Under consideration of the active center, the $q N$ was the only quenching coefficient altered by extended photoperiod in diploids. $q N$ reflecting the fraction of variable $\mathrm{Chl}$ fluorescence is quenched by nonphotochemical process (Lazár, 2015) rather than being used for photosynthetic electron transport (Genty et al., 1989). Our result of $\phi P S I I$ and QY_max on diploids was concomitant with this quenching data. In tetraploids, an enhanced quenching coefficient $q P$ and $q L$ suggested that the light excess from $16.5 \mathrm{~h}$ photoperiod was quenched into photochemistry, but a certain fraction absorbed light was dissipated into heat. The $q P$ (coefficient of photochemical quenching) indicated that excess light is quenched by photochemistry (Lazár, 2015 ) and reflected the degree of open reaction centers in which primary quinone aceptor $Q_{A}$ PSII is caplable of photoreduction (Krause and Weis, 1991). The $q L$ estimates nonphotochemical is formed only upon illumination to regulate the amount of absorbed light in avoiding damage effect (Kramer et al., 2004).

The pairwise comparison among ploidies in control treatments confirmed thathexaploid clone 29 and clone 35 performed a lower PQ than diploids. PQ of hexaploid clone 35 was also lower in stress treatment compared to diploids and tetraploids, which was probably due to the less optimal light intensity of the growth condition as suggested by $\phi P S I I$ and QY_max. The PQ parameter indicated the de-excitation pathway of absorbed light energy (Porcar-Castell et al., 2014). Hexaploid clone 29 also performed a lower $q_{l}$ than tetraploids in both treatments. Hence, we cannot make a general conclusion on hexaploids, but we rather detected a great variation in response to prolonged photoperiods, probably depending on the original habitat of the source population. The hexaploid clones/populations of this hybrid lineage are genetically all very similar, but nevertheless occupy a broad range of habitats from forest understory to forest margins and open meadows (Paun et al., 2006; Hörandl et al., 2009). 


\subsubsection{Fast fluorescence transient curve}

The OJIP curve illustrates the reduction of the acceptor side of PSII (represented as O-J phase), partial reduction of the PQ pool (represented as $\mathrm{J}-\mathrm{I}$ phase), and the reduction of the acceptor side of PSI (represented as I-P phase) (Yusuf et al., 2010; Ripoll et al., 2016). Our OJIP curves indicated that prolonged photoperiod altered the I-P phase in diploids, the J-I and I-P phase in tetraploids, all phases in hexaploid clone 35. Since the JIP-test parameters provide a more detailed characterization of fluorescence transient for screening the environmental effect on photosynthesis behavioral in plants e.g., Strasser et al. (2000), we extended the observation of the stress effect between diploids and tetraploids with JIP-tests.

The JIP-test between the treatments presented a stronger effect in diploids marked by a reduction of the value of $\mathrm{Pi}$ _Abs (performance index on absorption basis). Pi_Abs quantifies the overall performance of PSII electron flow (Stirbet et al., 2018). Prolonged photoperiod in diploids altered parameters of specific energy flux per reaction center $(\mathrm{RC})$, affecting the antenna molecules in the photosynthetic membrane, then resulted in higher closure of reaction center $(A B S / R C)$, reduced the capacity of photon trapping $\left(T R_{0} / R C\right)$; hence, photons dissipated to a higher extent $\left(\mathrm{DI}_{0} / \mathrm{RC}\right)$. Single turn-over $\left(\mathrm{S}_{\mathrm{S}}\right)$ represents the total amount of primary PSII acceptor $\left(Q_{A}\right)$ (Strasser et al., 2004) and corresponds to the O-J phase (Bordenave et al., 2019) as presented by a slightly higher O-J phase. The study of Rusaczonek et al. (2015) on Arabidopsis thaliana proposed that the inhibition of photosynthetic electron transport after UV stress might have originated from an increase in UV sensitivity of the light-dependent reactions (Phi_D $D_{0}$. In our study, the diploids in prolonged photoperiods might have an increased sensitivity of the light-dependent reactions. The specific energy fluxes of diploids were reduced, e.g., absorption flux per reaction center (RCs) (apparent antenna size of an active PSII) (ABS/RC), dissipated energy flux per $R C s\left(\mathrm{DI}_{0} / \mathrm{RC}\right)$, and trapped energy flux per $\mathrm{RCs}(\mathrm{TR} / \mathrm{RC})$. $\mathrm{ABS} / \mathrm{RC}$ estimates the ratio of the total number of photons absorbed by Chlorophyll of all reaction centers (RCs) in PSIl to the number of active RCs. In $A$. thaliana, the increase of $A B S / R C$ was an indication of inactivation of PSIl center after UV-C stress, a higher $T R_{0} / R C$ was an indication of reduction of plastoquinone $Q_{A}$ pool, and increasing of $\mathrm{DI}_{0} / \mathrm{RC}$ was an indication of PSII RCs damage and reduction of the efficiency in trapping photons (Rusaczonek et al., 2015). The stressed diploid Ranunculus auricomus plants presented a higher dissipated energy flux per reaction center $\left(\mathrm{DI}_{0} / \mathrm{RC}\right)$, which correlates with the result of higher dissipated heat (NPQ in this study) and fluorescence (Phi_P $\mathrm{P}_{0}$. A similar pattern was also reported in Çiçek et al. (2020) after UV-B stress in Scots pine, and the absorbed energy could not be directed to PSII for photochemistry but was dissipated as heat and fluorescence. 
In tetraploids, only a slight effect of prolonged photoperiod was observed by the reduction of $\mathrm{ET}_{0} / \mathrm{RC}$ and Fix.Area (area below the fluorescence curve between $\mathrm{F}_{20 \mu \mathrm{s}}$ and $\mathrm{F}_{1 \mathrm{~s}}$ ). Under stress treatment, the mean value of $\mathrm{ET}_{0} / \mathrm{RC}$ of tetraploid was lower than in the other cytotypes. $E T_{0} / R C$ was the later stage of electron transport per reaction center. The reduction of $E T_{0} / R C$ might indicate the blockage of PSIl electron transport beyond $Q_{A^{-}}$(Stirbet et al., 2018). Following Rusaczonek et al. (2015) that reduction of $E T_{0} / R C$ is related to an increase of inactive reaction centers of PSII, and hence in tetraploids, extended photoperiod might not affect the antenna molecules in the photosynthetic membrane as indicated by quenching coefficient $q P$, but exceeding the capacity of photon trapping $(\mathrm{TR} / \mathrm{RC})$; hence the excess light dissipated as non-photochemical quenching as indicated by quenching coefficient $q L$.

In hexaploids, again, the differentiation of the two clones was apparent. The hexaploid clone 35 performed a better photon trapping capacity $\left(\mathrm{TR}_{0} / \mathrm{RC}\right)$ and electron transport flux per $\mathrm{RCs}$ $\left(E T_{0} / R C\right)$ in the longer photoperiod. Specific energy fluxes parameter confirmed a higher photosynthesis performance of this clone and the PI_Abs indicating the requirement of higher light intensity for optimal photosynthesis. In clone 29, no differences appeared between treatments in any of the OJIP parameters. The variation of photosynthesis performance of hexaploids among two clones might relate to different habitats.

\subsection{Conclusion on ploidy-dependent light stress effects and on mode of reproduction}

Our study revealed differential responses of cytotypes in photosynthesis parameters to prolonged photoperiods. However, the response of photosynthesis performance is complex and differs in various parameters and does not follow a linear pattern of better stress tolerance with higher ploidy levels. Although we raised the plants under equal garden conditions, we suppose that different pre-adaptations from original habitats of the provenances may still influence the photosynthesis performance. Diploids originated from crosses of forest plants and hence might be preadapted to low light conditions (Hörandl et al., 2009). They respond to prolonged photoperiod in most photosynthesis parameters and tend to reduce excess energy via non-photochemical quenching. Tetraploids were raised from light-adapted meadow plants, and also the hexaploid clone 35 was a typical sun-adapted plant that originated from a meadow population with more sunlight exposure (Paun et al., 2006; Hörandl et al., 2009), clone 35 = VRU2), whereas hexaploid clone 29 (= TRE) originated from a half-shaded habitat at forest margins). Tetraploids showed altogether not much sensitivity to the change of photoperiod and appeared to be adapted to high light conditions. Hexaploids, however strongly differentiated according to their provenances. We suppose an influence of habitat as other factors are unlikely. All cytotypes originate from sites in Central Europe (at 47- 48 latitude and from the same altitudinal zone), and hence we can rather rule out that differential 
light intensities due to strong latitude (or altitude) gradients, as observed in Karbstein et al. (2021) over whole Europe, would influence the pattern. The variability of polyploid ecotypes may rely on a greater variance of gene expression patterns as they have highly heterozygous genomes (Karbstein et al., 2021). In tetraploid Glycine max, overexpression of oxidative stress-regulating genes compared to the diploid progenitors correlated to differential photosynthetic performance and adaptation to higher light intensities (Coate et al., 2012). Also, epigenetic control mechanisms may play a role. A study on cytosin-methylation of diploid and tetraploid $R$. kuepferi revealed not only different profiles between cytotypes but also indicated two different epigenetic groups within tetraploids, correlating to different temperature conditions (Schinkel et al., 2020).

The photosynthesis performance of $R$. auricomus cytotypes, however, does relate to mode of ovule formation, as diploids showed the highest sensitivity to prolonged photoperiod concomitant to the highest proportions of sexual ovules, followed by tetraploids (Ulum et al., 2020). Hexaploids, however, exhibited a very large variance in the proportions of sexual ovules, which we also observed here in photosynthesis performance. We detected here that this variation is mostly referable to two different ecotypes. We suppose that differential levels of oxidative stress influence the mode of reproduction, as in Boechera (Mateo de Arias et al., 2020). Different levels of oxidative stress in the reproductive tissues, however, need further investigation of metabolite profiles as important factors for the maintenance of redox homeodynamics.

Patents: Not applicable.

Author Contributions: $\mathrm{EH}$ and $\mathrm{FH}$ conceived and designed the study; FU performed the photo-synthesis analysis. Data were analyzed by $\mathrm{FU}$ and $\mathrm{FH}$, and $\mathrm{FU}, \mathrm{EH}$, and $\mathrm{FH}$ wrote and consented to the manuscript.

Funding: This project was founded by The German Research Fund DFG (DFG Hörandl Ho $43954-1)$ to $\mathrm{EH}$ and by the Indonesia endowment fund for education, grant no. PRJ2369/LPDP.3/2016 to FBU.

Data Availability Statement: The raw data are deposited at GöttingenResearchOnline (https://data.goettingen-research-online.de/dataset.xhtml?persistentld=doi:10.25625/ S7RGG7).

Acknowledgments: Silvia Friedrichs for nursing the plants; referees for valuable comments on the manuscript.

Conflicts of Interest: The authors declare no conflict of interest 
Supplementary Materials:

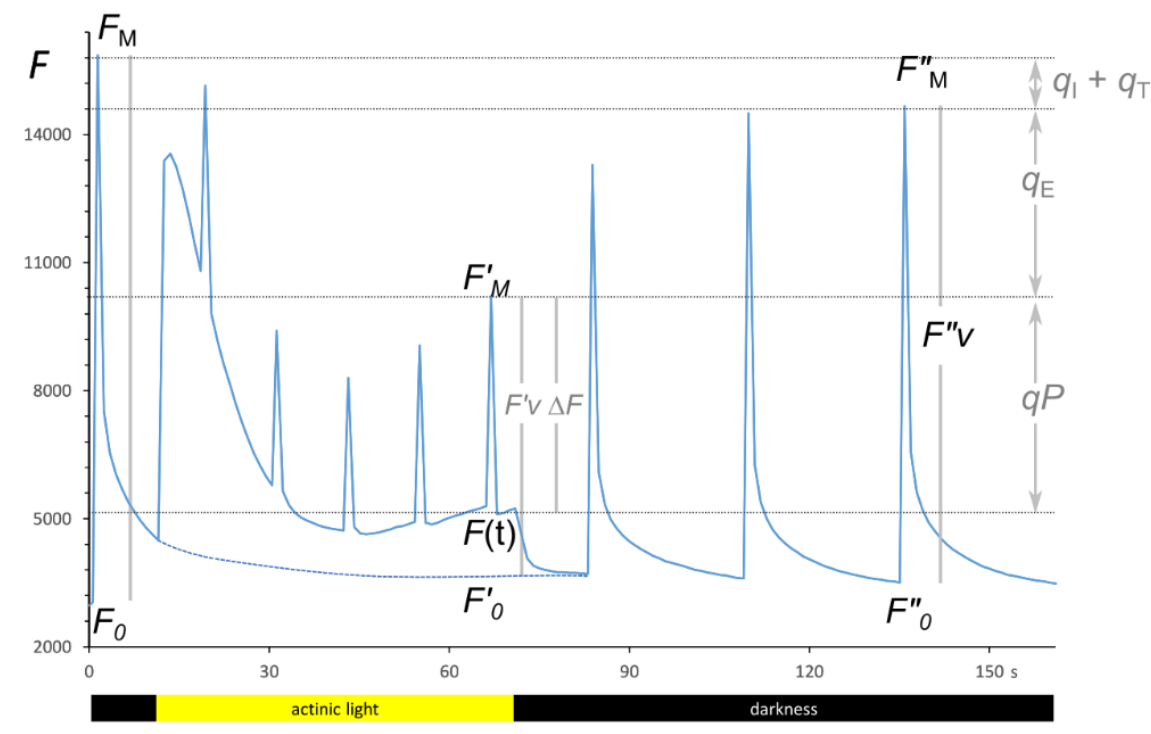

Figure S1: Induction curve and annotation of coefficients. 
Table S1. Plant material used in the treatments and for measurements of photosynthesis parameters.

\begin{tabular}{|c|c|c|c|c|c|c|c|}
\hline \multirow{2}{*}{ Ploidy } & \multirow{2}{*}{ Treatment } & \multirow{2}{*}{ Sample code } & \multirow{2}{*}{ Plant code } & \multicolumn{4}{|c|}{ Photosynthesis } \\
\hline & & & & PSII & ETR & IC & OJIP \\
\hline \multirow[t]{25}{*}{$2 x$} & Control & C2-12 & $\mathrm{J} 10 \times \mathrm{J} 30 / 03$ & $x$ & & & \\
\hline & & $\mathrm{C} 2-13$ & $\mathrm{~J} 10 \times \mathrm{J} 30 / 11$ & $x$ & $x$ & $x$ & $x$ \\
\hline & & $\mathrm{C} 2-15$ & $\mathrm{~J} 20 \mathrm{xJ} 2 / 16$ & $x$ & $x$ & $x$ & $x$ \\
\hline & & C2-19 & J24xJ22/12 & $x$ & $x$ & $x$ & $x$ \\
\hline & & C2-21 & F3xJ6/25 & $x$ & $x$ & $x$ & $x$ \\
\hline & & C2-24 & J10xJ14/18 & $x$ & & $x$ & $x$ \\
\hline & & $\mathrm{C} 2-25$ & J6xF3/19 & & $x$ & $x$ & $x$ \\
\hline & & $\mathrm{C} 2-26$ & J10xJ30/05 & $x$ & $x$ & & \\
\hline & & $\mathrm{C} 2-27$ & J6xF3/14 & & $x$ & $x$ & $x$ \\
\hline & & C2-4 & F3xJ6/28 & $x$ & & & \\
\hline & & $\mathrm{C} 2-7$ & J6xF3/14 & $x$ & $x$ & $x$ & $x$ \\
\hline & & C2-8 & J6xF7/12 & $x$ & $x$ & $x$ & $x$ \\
\hline & & C2-9 & J6xF7/14 & $x$ & $x$ & $x$ & $x$ \\
\hline & Stress & S2-1 & F3xJ6/01 & $x$ & $x$ & $x$ & $x$ \\
\hline & & $\mathrm{S} 2-18$ & $\mathrm{~J} 24 \mathrm{xJ} 22 / 03$ & $x$ & $x$ & $x$ & $x$ \\
\hline & & S2-2 & F3xJ6/04 & $x$ & $x$ & $x$ & $x$ \\
\hline & & S2-21 & F3xJ6/1 & $x$ & $x$ & $x$ & $x$ \\
\hline & & $\mathrm{S} 2-23$ & $\mathrm{~F} 10 \times \mathrm{J} 3 / 03$ & $x$ & $x$ & $x$ & $x$ \\
\hline & & S2-24 & $\mathrm{J} 6 \mathrm{xF} 3 / 06$ & $x$ & $x$ & $x$ & $x$ \\
\hline & & S2-25 & J10xJ14/09 & $x$ & & & \\
\hline & & S2-27 & J24xJ22/09 & $x$ & & & \\
\hline & & S2-3 & F3xJ6/05 & $x$ & $x$ & $x$ & $x$ \\
\hline & & S2-6 & $\mathrm{J} 6 \mathrm{xF} 3 / 02$ & $x$ & $x$ & $x$ & $x$ \\
\hline & & S2-7 & J6xF3/05 & $x$ & $x$ & $x$ & $x$ \\
\hline & & S2-9 & $\mathrm{J} 6 \mathrm{xF} 7 / 08$ & $x$ & $x$ & $x$ & $x$ \\
\hline \multirow[t]{11}{*}{$4 x$} & Control & C4-11 & LH1406030B4-08 & $x$ & $x$ & $x$ & $x$ \\
\hline & & C4-13 & LH1406030B4-16 & $x$ & $x$ & $x$ & $x$ \\
\hline & & C4-15 & LH1406030B4-18 & $x$ & $x$ & $x$ & $x$ \\
\hline & & C4-19 & LH1406030B5-07 & $x$ & $x$ & $x$ & $x$ \\
\hline & & C4-21 & LH1406030B5-16 & $x$ & $x$ & $x$ & $x$ \\
\hline & & C4-22 & LH1406030B5-17 & $x$ & $x$ & $x$ & $x$ \\
\hline & & C4-23 & LH1406030B5-19 & $x$ & $x$ & $x$ & $x$ \\
\hline & & C4-26 & LH4B005 & $x$ & & & \\
\hline & & C4-5 & LH1406030B2-04 & $x$ & $x$ & $x$ & $x$ \\
\hline & & C4-8 & LH1406030B4-02 & $x$ & $x$ & $x$ & $x$ \\
\hline & & C4-9 & LH1406030B4-05 & $x$ & $x$ & $x$ & $x$ \\
\hline
\end{tabular}




\begin{tabular}{|c|c|c|c|c|c|c|c|}
\hline \multirow{19}{*}{ Ploidy } & \multirow{2}{*}{ Treatment } & \multirow{2}{*}{ Sample code } & \multirow{2}{*}{ Plant code } & \multicolumn{4}{|c|}{ Photosynthesis } \\
\hline & & & & PSII & ETR & IC & OJIP \\
\hline & \multirow[t]{17}{*}{ Stress } & S4-11 & LH1406030B4-19 & $x$ & $x$ & $x$ & $x$ \\
\hline & & S4-14 & LH1406030B2-02 & $x$ & $x$ & $x$ & \\
\hline & & S4-14 & LH1406030B2-02 & $x$ & & & $x$ \\
\hline & & S4-16 & LH1406030B5-05 & $x$ & & & $x$ \\
\hline & & S4-2 & LH1406030B1-04 & $x$ & & $x$ & \\
\hline & & $S 4-20$ & LH1406030B5-13 & $x$ & & & $x$ \\
\hline & & S4-21 & LH1406030B5-18 & $x$ & $x$ & $x$ & \\
\hline & & S4-22 & LH1406030B5-20 & $x$ & & & \\
\hline & & S4-23 & LH1406030B4-10 & $x$ & $x$ & $x$ & $x$ \\
\hline & & S4-24 & LH1406030G1-8 & $x$ & & & $x$ \\
\hline & & S4-25 & LH1406030G1-16 & & $x$ & $x$ & $x$ \\
\hline & & S4-26 & LH1406030G1-18 & & $x$ & $x$ & \\
\hline & & S4-27 & LH1406030B5-04 & $x$ & & & \\
\hline & & S4-4 & LH1406030B2-01 & $x$ & & & $x$ \\
\hline & & S4-5 & LH1406030B2-07 & $x$ & $x$ & $x$ & \\
\hline & & S4-6 & LH1406030B4-01 & & $x$ & $x$ & $x$ \\
\hline & & S4-8 & LH1406030B4-11 & $x$ & $x$ & $x$ & \\
\hline \multirow[t]{8}{*}{$6 x$ clone 29} & \multirow[t]{5}{*}{ Control } & C6-1 & $29 / 15-3 N / 02$ & $x$ & $x$ & & \\
\hline & & C6-12 & $29 / 15-5 K / 21$ & $x$ & $x$ & $x$ & $x$ \\
\hline & & C6-5 & $29 / 15-5 K / 02$ & $x$ & $x$ & $x$ & $x$ \\
\hline & & C6-6 & $29 / 15-5 K / 05$ & $x$ & $x$ & $x$ & $x$ \\
\hline & & C6-7 & $29 / 15-5 K / 09$ & $x$ & $x$ & $x$ & $x$ \\
\hline & \multirow[t]{3}{*}{ Stress } & S6-39 & $29 / 15-5 K / 20$ & $x$ & $x$ & $x$ & $x$ \\
\hline & & S6-9 & $29 / 15-5 K / 06$ & $x$ & $x$ & $x$ & $x$ \\
\hline & & S6-1 & $29 / 15-5 K / 03$ & $x$ & $x$ & $x$ & $x$ \\
\hline \multirow[t]{16}{*}{$6 x$ clone 35} & \multirow[t]{7}{*}{ Control } & C6-15 & $35 / 28-4^{\star} / 26$ & $x$ & & & $x$ \\
\hline & & C6-16 & $35 / 28-4^{*} / 28$ & $x$ & $x$ & $x$ & $x$ \\
\hline & & C6-22 & $35 / 28-4 a / 16$ & $x$ & & & $x$ \\
\hline & & C6-23 & $35 / 28-4^{*} / 27$ & & $x$ & $x$ & $x$ \\
\hline & & C6-25 & $35 / 28-4 a / 22$ & $x$ & $x$ & $x$ & $x$ \\
\hline & & C6-33 & $35 / 28-4 Q / 82$ & $x$ & $x$ & & $x$ \\
\hline & & C6-15 & $35 / 28-4 * / 26$ & $x$ & $x$ & $x$ & \\
\hline & \multirow[t]{9}{*}{ Stress } & S6-17 & $35 / 28-4 * / 03$ & $x$ & $x$ & $x$ & $x$ \\
\hline & & S6-19 & $35 / 28-4^{*} / 18$ & $x$ & $x$ & $x$ & $x$ \\
\hline & & S6-21 & $35 / 28-4^{*} / 24$ & & $x$ & $x$ & $x$ \\
\hline & & S6-22 & $35 / 28-4 * / 22$ & & & $x$ & \\
\hline & & S6-24 & $35 / 28-4^{*} / 26$ & & $x$ & $x$ & $x$ \\
\hline & & S6-25 & $35 / 28-4^{*} / 40$ & & $x$ & $x$ & $x$ \\
\hline & & S6-29 & $35 / 28-4 Q / 27$ & $x$ & & $x$ & \\
\hline & & S6-33 & $35 / 28-4 Q / 28$ & $x$ & & $x$ & \\
\hline & & S6-37 & $35 / 28-4 a / 40$ & & & $x$ & $x$ \\
\hline
\end{tabular}


Table S2. Formulae and definitions of terms used in photosynthesis parameters (Strasser and Govindjee, 1992; Strasser et al., 2004; Baker, 2008; Tsimilli-Michael and Strasser, 2013; Lazár, 2015; Rusaczonek et al., 2015)

\begin{tabular}{|c|c|}
\hline Parameter formula & Explanation \\
\hline$\phi P S I I=F^{\prime} V / F^{\prime} M$ & PSII maximum efficiency \\
\hline QY_max $=F v / F M$ & $\begin{array}{l}\text { maximum quantum efficiency of PSII } \\
\text { photochemistry }\end{array}$ \\
\hline $\mathrm{ETR}=\phi \mathrm{PSII} \times \mathrm{PPFD} \times 0.5$ & $\begin{array}{l}\text { Electron transport rate; PPFD: Photosynthetic } \\
\text { photon flux density }\end{array}$ \\
\hline $\begin{array}{l}\text { Fluorescence quenching analysis of induction } \\
\text { curve }\end{array}$ & \\
\hline$P Q=(F M / F(t))-\left(F M / F^{\prime} M\right)$ & Photochemical quenching (PQ) \\
\hline$N P Q=\left(F M / F^{\prime} M\right) / F^{\prime} M$ & Non-photochemical quenching (NPQ) \\
\hline $\mathrm{qE}=\left(\mathrm{F}^{\prime \prime} \mathrm{M}-\mathrm{F}^{\prime} \mathrm{V}\right) / \mathrm{F}^{\prime \prime} \mathrm{M}$ & $\begin{array}{l}\text { Energy-dependent non-photochemical quenching } \\
\text { coefficient }\end{array}$ \\
\hline$q \mathrm{q}=1-(\mathrm{F} " \mathrm{~V} / \mathrm{FV})$ & $\begin{array}{l}\text { Photoinhibitory non-photochemical quenching } \\
\text { coefficient }\end{array}$ \\
\hline$N P Q E=\left(F M / F^{\prime} M\right)-\left(F M / F^{\prime \prime} M\right)$ & $\begin{array}{l}\text { Energy-dependent non-photochemical quenching } \\
\text { coefficient }\end{array}$ \\
\hline$N P Q I=(F V / F “ V)-1$ & Photoinhibitory non-photochemical quenching \\
\hline $\mathrm{qP}=\Delta \mathrm{F} /\left(\mathrm{F}^{\prime} \mathrm{M}-\mathrm{F}^{\prime} 0\right)$ & coefficient of photochemical quenching \\
\hline $\mathrm{qN}=\left(\mathrm{F}^{\prime} \mathrm{M}-\mathrm{F}^{\prime} 0\right) / \mathrm{Fv}$ & Non-photochemical quenching coefficient \\
\hline $\mathrm{qL}=\left(\left(\mathrm{F}^{\prime} \mathrm{M}-\mathrm{F}(\mathrm{t})\right) /\left(\mathrm{F}^{\prime} \mathrm{M}-\mathrm{F}^{\prime} 0\right)\right)^{\star}\left(\mathrm{F}^{\prime} 0 / \mathrm{F}(\mathrm{t})\right)$ & $\begin{array}{l}\text { Estimates the fraction of "open" PSII centers (lake } \\
\text { model) }\end{array}$ \\
\hline \multicolumn{2}{|l|}{ JIP-test parameter } \\
\hline $\mathrm{F} 0=\mathrm{F} 50 \mu \mathrm{s}$ & Fluorescence intensity at $50 \mu \mathrm{s}$ \\
\hline $\mathrm{Fj}$ & Fluorescence intensity at J-step (at 2ms) \\
\hline $\mathrm{Fi}$ & Fluorescence intensity at I-step (at $30 \mathrm{~ms}$ ) \\
\hline FM & Maximum fluorescence intensity \\
\hline $\mathrm{Fv}=\mathrm{FM}-\mathrm{F} 0$ & Maximal variable fluorescence \\
\hline FM/F0 & Ratio of fluorescence \\
\hline $\mathrm{FV} / \mathrm{F} 0$ & Efficiency of the oxygen-evolving complex \\
\hline Area & $\begin{array}{l}\text { Area between fluorescence curve and FM } \\
\text { (background subtracted) }\end{array}$ \\
\hline Fix.Area & $\begin{array}{l}\text { Area below the fluorescence curve between } \\
\text { F20 } \mu \text { s and F1s (background subtracted) }\end{array}$ \\
\hline $\mathrm{M} 0=\mathrm{TR} 0 / \mathrm{RC}-\mathrm{ET} 0 / \mathrm{RC}=4(\mathrm{~F} 300-\mathrm{F} 0) /(\mathrm{FM}-\mathrm{F} 0)$ & $\begin{array}{l}\text { Approximated initial slope (in } \mathrm{ms}-1 \text { ) of the } \\
\text { fluorescence transient normalized on the maximal } \\
\text { variable fluorescence FV }\end{array}$ \\
\hline $\mathrm{SM}=$ Area $/(\mathrm{FM}-\mathrm{F} 0)$ & The normalized area above the OJIP curve \\
\hline Ss & The smallest SM turn-over (single turn-over) \\
\hline $\mathrm{N}=\mathrm{SM} \times \mathrm{M} 0 \times(1 / \mathrm{Vj})$ & Turn-over number QA \\
\hline Phi_P0 = 1 - (F0/FM) (or FV/FM) & $\begin{array}{l}\text { Maximum quantum yield of primary } \\
\text { photochemistry }\end{array}$ \\
\hline $\mathrm{Vi}=(\mathrm{Fi}-\mathrm{F} 0) /(\mathrm{FM}-\mathrm{F} 0)$ & Relative variable fluorescence at the I-step \\
\hline$V j=(F j-F 0) /(F M-F 0)$ & Relative variable fluorescence at the $\mathrm{J}$-step \\
\hline Phi_D0 = 1- Phi_P0 - (F0 - FM) & Quantum yield of energy dissipation \\
\hline Phi_E0 $=(1-($ F0/FM $)) \times$ Psi_0 & Quantum yield of electron transport at time zero \\
\hline Phi_P0 = 1 - (F0/FM) (or FV/FM) & $\begin{array}{l}\text { Maximum quantum yield of primary } \\
\text { photochemistry }\end{array}$ \\
\hline
\end{tabular}




\begin{tabular}{|c|c|}
\hline Parameter formula & Explanation \\
\hline Psi_0 = 1-Vj & $\begin{array}{l}\text { Probability that a trapped exciton moves an } \\
\text { electron into the electron transport chain beyond } \\
\text { QA- }\end{array}$ \\
\hline $\begin{array}{l}\text { Phi_Pav }=\text { Phi_P0 }(\mathrm{SM} / \mathrm{tFM}) \\
\text { tFM = time to reach Fm (in ms) }\end{array}$ & $\begin{array}{l}\text { Time to reach maximum chlorophyll fluorescence } \\
\text { level (in } \mathrm{ms} \text { ) }\end{array}$ \\
\hline \multicolumn{2}{|l|}{ Specific energy fluxes (per active PSII): } \\
\hline$A B S / R C=(M 0 / V j) / P h i \_P 0$ & $\begin{array}{l}\text { Absorption flux per reaction center (RC) (apparent } \\
\text { antenna size of an active PSII) }\end{array}$ \\
\hline $\mathrm{TR} 0 / \mathrm{RC}=\mathrm{M} 0 / \mathrm{Vj}$ & Trapped energy flux per RC \\
\hline$E T 0 / R C=(M 0 / V j) \times$ Psi_0 & Electron transport flux per reaction center (RC) \\
\hline $\mathrm{DIO} / \mathrm{RC}=\mathrm{ABS} / \mathrm{RC}-\mathrm{TR} 0 / \mathrm{RC}$ & Dissipated energy flux per reaction center (RC) \\
\hline $\begin{array}{l}\text { PI_Abs }= \\
(\text { RC/ABS }) \times \text { Phi_P }\end{array}$ & $\begin{array}{l}\text { Performance index on absorption basis related to } \\
\text { the overall photosynthetic activity of PSII }\end{array}$ \\
\hline
\end{tabular}

References:

Baker, N.R. (2008). Chlorophyll fluorescence: a probe of photosynthesis in vivo. Annu. Rev. Plant Biol. 59, 89-113.

Lazár, D. (2015). Parameters of photosynthetic energy partitioning. Journal of Plant Physiology 175, 131-147.

Rusaczonek, A., Czarnocka, W., Kacprzak, S., Witoń, D., Ślesak, I., Szechyńska-Hebda, M., et al. (2015). Role of phytochromes $A$ and $B$ in the regulation of cell death and acclimatory responses to UV stress in Arabidopsis thaliana. Journal of experimental botany 66(21), 6679-6695.

Strasser, R.J., and Govindjee (1992). "The Fo and the O-J-I-P Fluorescence Rise in Higher Plants and Algae," in Regulation of Chloroplast Biogenesis, ed. J.H. ArgyroudiAkoyunoglou. (Boston, MA: Springer US), 423-426.

Strasser, R.J., Tsimilli-Michael, M., and Srivastava, A. (2004). "Analysis of the chlorophyll a fluorescence transient," in Chlorophyll a fluorescence. Springer), 321-362.

Tsimilli-Michael, M., and Strasser, R.J. (2013). The energy flux theory 35 years later: formulations and applications. Photosynthesis research 117(1), 289-320. 
Table S3. Summary statistics of PSII maximum efficiency (ФPSII) and maximum quantum efficiency of PSIl photochemistry (QY_max) between treatment and among different ploidies and hexaploid clones. $\underline{\mathrm{n}}=$ number of measurements from $9-14$ individual per group.

\begin{tabular}{|c|c|c|c|c|c|c|c|c|c|}
\hline \multirow{2}{*}{ Ploidy } & \multirow{2}{*}{ Treatment } & \multicolumn{4}{|c|}{ ФPSII } & \multicolumn{4}{|c|}{ QY_max } \\
\hline & & $\mathbf{n}$ & mean & sd & median & $\mathbf{n}$ & mean & sd & median \\
\hline \multirow[b]{2}{*}{$2 x$} & Control & 130 & 0.721 & 0.028 & 0.729 & 30 & 0.819 & 0.03 & 0.83 \\
\hline & Stress & 131 & 0.710 & 0.027 & 0.713 & 30 & 0.816 & 0.015 & 0.817 \\
\hline \multirow{2}{*}{$4 x$} & Control & 121 & 0.707 & 0.024 & 0.710 & 30 & 0.823 & 0.016 & 0.825 \\
\hline & Stress & 99 & 0.700 & 0.037 & 0.711 & 30 & 0.817 & 0.021 & 0.82 \\
\hline \multirow{2}{*}{ 6x_29 } & Control & 45 & 0.720 & 0.026 & 0.725 & 13 & 0.828 & 0.009 & 0.83 \\
\hline & Stress & 33 & 0.722 & 0.031 & 0.725 & 9 & 0.808 & 0.027 & 0.811 \\
\hline \multirow{2}{*}{ 6x_35 } & Control & 55 & 0.628 & 0.068 & 0.631 & 16 & 0.705 & 0.08 & 0.702 \\
\hline & Stress & 60 & 0.675 & 0.041 & 0.675 & 21 & 0.664 & 0.088 & 0.642 \\
\hline
\end{tabular}


Table S4. Summary of generalized linear mixed models analysis for change in PSII maximum efficiency (ФPSII) to factors treatment and ploidy level (hexaploid separated in to two clones).

\begin{tabular}{llllll} 
& Estimate & Std. Error & $\mathbf{z}$ value & $\operatorname{Pr}(>|\mathbf{z}|)$ & \\
\hline Intercept & 0.94215 & 0.03053 & 30.859 & $<0.000001$ & $* * *$ \\
Stress treatment & -0.04569 & 0.04318 & -1.058 & 0.29 & \\
4x & -0.06073 & 0.04337 & -1.400 & 0.161 & \\
6x_29 & 0.00058 & 0.05910 & 0.010 & 0.992 & \\
6x_35 & -0.46562 & 0.05185 & -8.980 & $<0.0000001$ & $* * *$ \\
Stress:4x & -0.00401 & 0.06045 & -0.066 & 0.947 & \\
Stress:6x_29 & 0.05802 & 0.08860 & 0.655 & 0.513 & \\
Stress:6x_35 & 0.30527 & 0.07169 & 4.258 & 0.00002 & $* * *$ \\
\hline
\end{tabular}


Table S5. Pairwise comparison of PSII maximum efficiency (DPSII) among different ploidies and hexaploid clones of each treatment. Significant results are in bold.

\begin{tabular}{|c|c|c|c|c|c|c|c|c|c|}
\hline \multirow{2}{*}{\multicolumn{2}{|c|}{ Contrast }} & \multicolumn{4}{|c|}{ Control } & \multicolumn{4}{|c|}{ Stress } \\
\hline & & estimate & SE & t.ratio & p.value & estimate & SE & t.ratio & p.value \\
\hline $2 x$ & $4 x$ & 0.06 & 0.04 & 1.40 & 0.85050 & 0.06 & 0.04 & 1.54 & 0.77740 \\
\hline $2 x$ & $6 x \_29$ & 0.00 & 0.06 & -0.01 & 1.00000 & -0.06 & 0.07 & -0.89 & 0.98630 \\
\hline $2 x$ & 6x_35 & 0.47 & 0.05 & 8.98 & $<.0001$ & 0.16 & 0.05 & 3.24 & 0.02570 \\
\hline $4 x$ & $6 x \_29$ & -0.06 & 0.06 & -1.04 & 0.96710 & -0.12 & 0.07 & -1.89 & 0.54690 \\
\hline $4 x$ & $6 x \_35$ & 0.40 & 0.05 & 7.78 & $<.0001$ & 0.10 & 0.05 & 1.97 & 0.49150 \\
\hline $6 x \_29$ & $6 x \_35$ & 0.47 & 0.07 & 7.10 & $<.0001$ & 0.22 & 0.07 & 3.11 & 0.03810 \\
\hline
\end{tabular}


Table S6. Summary of generalized linear mixed model analysis for change in maximum quantum efficiency of PSII photochemistry (QY_max) to factors treatment and ploidy level (hexaploid separated in to two clones).

\begin{tabular}{llllll}
\hline & Estimate & Std. Error & $\mathbf{z}$ value & $\operatorname{Pr}(>|\mathbf{z}|)$ & \\
\hline Intercept & 1.52182 & 0.05718 & 26.61300 & $<0.000001$ & $* * *$ \\
Stress treatment & -0.03586 & 0.08185 & -0.43800 & 0.66100 & \\
$4 \mathrm{x}$ & 0.01244 & 0.08201 & 0.15200 & 0.87900 & \\
6x_29 & 0.03794 & 0.10358 & 0.36600 & 0.71400 & \\
6x_35 & -0.64995 & 0.09362 & -6.94200 & 0.00000 & $* * *$ \\
Stress:4x & 0.00751 & 0.11378 & 0.06600 & 0.94700 & \\
Stress:6x_29 & -0.08429 & 0.15974 & -0.52800 & 0.59800 & \\
Stress:6x_35 & -0.12520 & 0.12657 & -0.98900 & 0.32300 & \\
\hline
\end{tabular}


Table S7. Pairwise comparisons of QY_max among different ploidies and hexaploid clones of each treatment. Significant results are in bold.

\begin{tabular}{|c|c|c|c|c|c|c|c|c|c|c|}
\hline \multirow{2}{*}{\multicolumn{2}{|c|}{ Contrast }} & \multicolumn{4}{|c|}{ Control } & \multicolumn{5}{|c|}{ Stress } \\
\hline & & \multirow{2}{*}{$\begin{array}{l}\text { estimate } \\
-0.01\end{array}$} & \multirow{2}{*}{$\begin{array}{r}\text { SE } \\
0.08\end{array}$} & \multirow{2}{*}{$\begin{array}{c}\text { df t.ratio } \\
169-0.15\end{array}$} & \multirow{2}{*}{$\begin{array}{l}\text { p.value } \\
1.000\end{array}$} & \multirow{2}{*}{$\begin{array}{l}\text { estimate } \\
-0.02\end{array}$} & \multirow{2}{*}{$\begin{array}{r}\text { SE } \\
0.08\end{array}$} & \multirow{2}{*}{$\begin{array}{r}\text { df } \\
169\end{array}$} & \multirow{2}{*}{$\begin{array}{l}\text { t.ratio } \\
-0.25\end{array}$} & \multirow{2}{*}{$\frac{\text { p.value }}{1.000}$} \\
\hline $2 x$ & $4 x$ & & & & & & & & & \\
\hline $2 x$ & $6 x \_29$ & -0.04 & 0.10 & $169-0.37$ & 1.000 & 0.05 & 0.12 & 169 & 0.38 & 1.000 \\
\hline $2 x$ & $6 x \_35$ & 0.65 & 0.09 & 1696.94 & $<.0001$ & 0.78 & 0.09 & 169 & 9.10 & $<.0001$ \\
\hline $4 x$ & $6 x \_29$ & -0.03 & 0.10 & $169-0.24$ & 1.000 & 0.07 & 0.12 & 169 & 0.56 & 0.999 \\
\hline $4 x$ & $6 x \_35$ & 0.40 & 0.05 & 6647.78 & $<.0001$ & 0.80 & 0.08 & 169 & 9.78 & $<.0001$ \\
\hline $6 x+29$ & $6 x \_35$ & 0.66 & 0.09 & 1697.00 & $<.0001$ & 0.73 & 0.12 & 169 & 5.92 & $<.0001$ \\
\hline
\end{tabular}


Table S8. Pairwise comparison of relative electron transport rate among different ploidies and hexaploid clones of each treatment under different light intensities (10-500). Significant results ( $p$ adj. sign.) are in bold.

\begin{tabular}{|c|c|c|c|c|c|c|c|c|}
\hline \multirow{2}{*}{ PPFD } & \multirow{2}{*}{ Ploidy } & & \multicolumn{3}{|c|}{ Control } & \multicolumn{3}{|c|}{ Stress } \\
\hline & & & statistic & $p$ & p.adj.signif & statistic & $p$ & p.adj.signif \\
\hline \multirow[t]{6}{*}{10} & $2 x$ & $4 x$ & 68.000 & 0.185 & 1.0000 & 57.500 & 0.322 & 1.0000 \\
\hline & $2 x$ & $6 x \_29$ & 28.500 & 0.711 & 1.0000 & 26.500 & 0.062 & 0.3710 \\
\hline & $2 x$ & 6x_35 & 45.000 & 0.017 & 0.1000 & 50.000 & 0.003 & 0.0160 \\
\hline & $4 x$ & $6 x \_29$ & 17.000 & 0.357 & 1.0000 & 23.000 & 0.093 & 0.5560 \\
\hline & $4 x$ & $6 x \_35$ & 34.500 & 0.269 & 1.0000 & 45.000 & 0.003 & 0.0190 \\
\hline & $6 x+29$ & $6 x \_35$ & 24.000 & 0.020 & 0.1180 & 14.000 & 0.067 & 0.4010 \\
\hline \multirow[t]{6}{*}{20} & $2 x$ & $4 x$ & 63.500 & 0.324 & 1.0000 & 43.500 & 0.934 & 1.0000 \\
\hline & $2 x$ & $6 x \_29$ & 27.500 & 0.805 & 1.0000 & 27.000 & 0.050 & 0.3020 \\
\hline & $2 x$ & $6 x \_35$ & 46.000 & 0.012 & 0.0710 & 50.000 & 0.003 & 0.0160 \\
\hline & $4 x$ & $6 x \_29$ & 15.000 & 0.242 & 1.0000 & 24.000 & 0.060 & 0.3620 \\
\hline & $4 x$ & $6 x \_35$ & 45.000 & 0.016 & 0.0970 & 45.000 & 0.003 & 0.0190 \\
\hline & $6 x \_29$ & 6x_35 & 25.000 & 0.011 & 0.0670 & 15.000 & 0.036 & 0.2150 \\
\hline \multirow[t]{6}{*}{50} & $2 x$ & $4 x$ & 67.000 & 0.211 & 1.0000 & 56.500 & 0.366 & 1.0000 \\
\hline & $2 x$ & $6 x \_29$ & 34.500 & 0.267 & 1.0000 & 24.500 & 0.123 & 0.7380 \\
\hline & $2 x$ & 6x_35 & 47.000 & 0.008 & 0.0500 & 50.000 & 0.003 & 0.0150 \\
\hline & $4 x$ & $6 x \_29$ & 20.000 & 0.577 & 1.0000 & 22.500 & 0.110 & 0.6600 \\
\hline & $4 x$ & 6x_35 & 47.000 & 0.008 & 0.0490 & 45.000 & 0.003 & 0.0190 \\
\hline & 6x_29 & 6x_35 & 25.000 & 0.012 & 0.0700 & 15.000 & 0.036 & 0.2140 \\
\hline \multirow[t]{6}{*}{100} & $2 x$ & $4 x$ & 66.500 & 0.225 & 1.0000 & 55.500 & 0.412 & 1.0000 \\
\hline & $2 x$ & 6x_29 & 37.500 & 0.140 & 0.8400 & 22.500 & 0.233 & 1.0000 \\
\hline & $2 x$ & $6 x \_35$ & 43.000 & 0.032 & 0.1900 & 46.500 & 0.010 & 0.0590 \\
\hline & $4 x$ & 6x_29 & 24.000 & 0.951 & 1.0000 & 19.500 & 0.303 & 1.0000 \\
\hline & $4 x$ & 6x_35 & 45.500 & 0.014 & 0.0850 & 44.500 & 0.004 & 0.0240 \\
\hline & $6 x \_29$ & 6x_35 & 25.000 & 0.012 & 0.0700 & 15.000 & 0.036 & 0.2140 \\
\hline \multirow[t]{6}{*}{300} & $2 x$ & $4 x$ & 82.000 & 0.017 & 0.1010 & 76.000 & 0.012 & 0.0730 \\
\hline & $2 x$ & $6 x \_29$ & 30.500 & 0.539 & 1.0000 & 17.500 & 0.732 & 1.0000 \\
\hline & $2 x$ & 6x_35 & 40.000 & 0.075 & 0.4500 & 41.000 & 0.054 & 0.3260 \\
\hline & $4 x$ & $6 x \_29$ & 2.000 & 0.006 & 0.0330 & 0.000 & 0.016 & 0.0930 \\
\hline & $4 x$ & 6x_35 & 21.500 & 0.708 & 1.0000 & 22.000 & 1.000 & 1.0000 \\
\hline & 6x_29 & 6x_35 & 24.500 & 0.016 & 0.0940 & 15.000 & 0.035 & 0.2080 \\
\hline \multirow[t]{6}{*}{500} & $2 x$ & $4 x$ & 82.500 & 0.015 & 0.0910 & 78.000 & 0.008 & 0.0460 \\
\hline & $2 x$ & 6x_29 & 25.000 & 1.000 & 1.0000 & 5.500 & 0.117 & 0.7020 \\
\hline & $2 x$ & $6 x \_35$ & 32.500 & 0.387 & 1.0000 & 34.500 & 0.264 & 1.0000 \\
\hline & $4 x$ & 6x_29 & 0.000 & 0.003 & 0.0160 & 0.000 & 0.016 & 0.0940 \\
\hline & $4 x$ & 6x_35 & 3.500 & 0.010 & 0.0580 & 8.500 & 0.070 & 0.4180 \\
\hline & 6x_29 & 6x_35 & 24.000 & 0.019 & 0.1150 & 15.000 & 0.035 & 0.2080 \\
\hline
\end{tabular}


Table S9. Summary statistics and $P$ - values of Wilcoxon-Mann-Whitney-Test for determination of effects of prolonged photoperiod on induction curves parameters between treatments. S-C: proportion of alternation of the mean value, calculated as (Stress - Control/(Stress + Control)*100. Significant results are in bold

\begin{tabular}{|c|c|c|c|c|c|c|c|c|c|}
\hline \multirow{2}{*}{ Parameter } & \multirow{2}{*}{ Ploidy } & \multicolumn{3}{|c|}{ Control treatment $(\mathrm{C})$} & \multicolumn{3}{|c|}{ Stress treatment (C) } & \multirow{2}{*}{ S-C } & \multirow{2}{*}{$\mathbf{p}$} \\
\hline & & $\mathbf{n}$ & mean & sd & $\mathbf{n}$ & mean & sd & & \\
\hline \multirow[t]{4}{*}{$\overline{F v}$} & $2 x$ & 10 & 11770 & 2725.722 & 10 & 12189.4 & 1169.17 & 1.75 & 0.912 \\
\hline & $4 x$ & 10 & 12835.5 & 2900.151 & 10 & 11953.8 & 1696.967 & -3.56 & 0.165 \\
\hline & $6 x \_29$ & 4 & 14050.25 & 1202.819 & & 4311.67 & 779.973 & 0.92 & 1 \\
\hline & $6 x \_35$ & 4 & 9874 & 3098.879 & & 624.778 & 2099.756 & -1.28 & 0.94 \\
\hline \multirow[t]{4}{*}{$\overline{F v^{\prime}}$} & $2 x$ & 10 & 6605.592 & 2261.851 & 105 & 403.539 & 1376.386 & -10.01 & 0.165 \\
\hline & $4 x$ & 10 & 5423.215 & 2000.658 & 104 & 496.728 & 1119.035 & -9.34 & 0.218 \\
\hline & $6 x \_29$ & 4 & 9071.828 & 1417.599 & & 7190.53 & 506.356 & -11.57 & 0.229 \\
\hline & $6 x \_35$ & 4 & 3878.085 & 2756.17 & & 279.352 & 1252.866 & -8.37 & 0.94 \\
\hline \multirow[t]{4}{*}{$\overline{F v "}$} & $2 x$ & 10 & 9854.2 & 2627.07 & 10 & 10056 & 1379.529 & 1.01 & 0.623 \\
\hline & $4 x$ & 10 & 9522.3 & 2394.021 & 10 & 9224.7 & 1420.841 & -1.59 & 0.436 \\
\hline & $6 x \_29$ & 4 & 12358 & 921.55 & 3 & 12436 & 673.143 & 0.31 & 1 \\
\hline & $6 x \_35$ & 4 & 7547 & 3681.889 & & 168.222 & 1717.041 & -2.57 & 0.825 \\
\hline \multirow[t]{4}{*}{$\overline{F(t)}$} & $2 x$ & 10 & 5085.4 & 1422.407 & 10 & 4500.6 & 954.277 & -6.10 & 0.247 \\
\hline & $4 x$ & 10 & 5098.8 & 1740.582 & 10 & 4129.4 & 752.516 & -10.50 & 0.0892 \\
\hline & $6 x \_29$ & 4 & 7709.25 & 1707.153 & & 413.333 & 476.708 & -9.18 & 0.229 \\
\hline & $6 x \_35$ & 4 & 4896.75 & 2058.913 & 9 & 4542 & 741.238 & -3.76 & 0.71 \\
\hline \multirow[t]{4}{*}{$\overline{F 0}$} & $2 x$ & 10 & 2698.9 & 655.178 & 10 & 2718 & 231.407 & 0.35 & 0.705 \\
\hline & $4 x$ & 10 & 2622.1 & 582.257 & 10 & 2693.3 & 467.979 & 1.34 & 1 \\
\hline & 6x_29 & 4 & 2801.75 & 118.829 & & 3278.667 & 44.106 & 7.84 & 0.0571 \\
\hline & $6 x \_35$ & 4 & 4114.75 & 1468.216 & 9 & 4352 & 987.434 & 2.80 & 0.71 \\
\hline \multirow[t]{4}{*}{$\overline{\text { F0' }}$} & $2 x$ & 10 & 2389.208 & 522.211 & 102 & 310.361 & 256.488 & -1.68 & 0.481 \\
\hline & $4 x$ & 10 & 2206.885 & 479.607 & 102 & 180.172 & 331.323 & -0.61 & 0.579 \\
\hline & $6 x \_29$ & 4 & 2605.422 & 132.82 & & 2877.47 & 66.768 & 4.96 & 0.0571 \\
\hline & $6 x \_35$ & 4 & 2920.165 & 486.316 & & 1112.537 & 556.611 & 3.19 & 0.71 \\
\hline \multirow[t]{4}{*}{ F0" } & $2 x$ & 10 & 2959.2 & 776.997 & 10 & 2965.1 & 324.253 & 0.10 & 0.912 \\
\hline & $4 x$ & 10 & 3137.7 & 718.385 & 10 & 3030.5 & 521.163 & -1.74 & 0.307 \\
\hline & $6 x \_29$ & 4 & 3280 & 29.439 & & 3675.333 & 54.501 & 5.68 & 0.0571 \\
\hline & $6 x \_35$ & 4 & 4325 & 804.503 & & 726.222 & 935.938 & 4.43 & 0.604 \\
\hline \multirow[t]{4}{*}{$\overline{F M}$} & $2 x$ & 10 & 14468.9 & 3023.335 & 10 & 14907.4 & 1255.799 & 1.49 & 1 \\
\hline & $4 x$ & 10 & 15457.6 & 3457.08 & 10 & 14647.1 & 1985.781 & -2.69 & 0.241 \\
\hline & $6 x \_29$ & 4 & 16852 & 1295.326 & & 7590.33 & 808.047 & 2.14 & 0.629 \\
\hline & $6 x \_35$ & 4 & 13988.75 & 2801.748 & & 3976.78 & 1935.453 & -0.04 & 0.817 \\
\hline \multirow[t]{4}{*}{ FM' } & $2 x$ & 10 & 8994.8 & 2502.283 & 10 & 7713.9 & 1574.58 & -7.67 & 0.143 \\
\hline & $4 x$ & 10 & 7630.1 & 2339.183 & 10 & 6676.9 & 1275.26 & -6.66 & 0.247 \\
\hline & $6 x \_29$ & 4 & 11677.25 & 1523.304 & 3 & 10068 & 570.071 & -7.40 & 0.229 \\
\hline & $6 x \_35$ & 4 & 6798.25 & 2672.861 & & 391.889 & 1322.82 & -3.08 & 0.825 \\
\hline
\end{tabular}




\begin{tabular}{|c|c|c|c|c|c|c|c|c|c|}
\hline \multirow{2}{*}{ Parameter } & \multirow{2}{*}{ Ploidy } & \multicolumn{3}{|c|}{ Control treatment $(\mathrm{C})$} & \multicolumn{3}{|c|}{ Stress treatment (C) } & \multirow{2}{*}{ S-C } & \multirow{2}{*}{$\mathbf{p}$} \\
\hline & & $\mathbf{n}$ & mean & sd & $\mathbf{n}$ & mean & sd & & \\
\hline \multirow[t]{4}{*}{ FM" } & $2 x$ & 10 & 12813.4 & 3032.888 & 10 & 13021.1 & 1587.794 & 0.80 & 0.796 \\
\hline & $4 x$ & 10 & 12660 & 3036.018 & 10 & 12255.2 & 1678.656 & -1.62 & 0.315 \\
\hline & $6 x \_29$ & 4 & 15638 & 920.906 & & 6111.33 & 681.414 & 1.49 & 0.629 \\
\hline & $6 x \_35$ & 4 & 11872 & 3116.23 & & 1894.44 & 1568.051 & 0.09 & 0.643 \\
\hline \multirow[t]{4}{*}{$\Delta \mathrm{F}$} & $2 x$ & 10 & 3909.4 & 1204.87 & 10 & 3213.3 & 740.585 & -9.77 & 0.112 \\
\hline & $4 x$ & 10 & 2531.3 & 713.406 & 10 & 2547.5 & 643.174 & 0.32 & 1 \\
\hline & $6 x \_29$ & 4 & 3968 & 568.674 & & 3654.667 & 99.485 & -4.11 & 0.857 \\
\hline & $6 x \_35$ & 4 & 1901.5 & 678.819 & & 849.889 & 655.695 & -1.38 & 1 \\
\hline \multirow[t]{4}{*}{ NPQ } & $2 x$ & 10 & 0.654 & 0.213 & 10 & 0.99 & 0.347 & 20.44 & 0.0185 \\
\hline & $4 x$ & 10 & 1.102 & 0.39 & 10 & 1.241 & 0.376 & 5.93 & 0.631 \\
\hline & $6 x \_29$ & 4 & 0.451 & 0.088 & 3 & 0.753 & 0.173 & 25.08 & 0.0571 \\
\hline & $6 x \_35$ & 4 & 1.164 & 0.451 & 9 & 1.237 & 0.381 & 3.04 & 0.71 \\
\hline \multirow[t]{4}{*}{$\overline{P Q}$} & $2 x$ & 10 & 1.263 & 0.238 & 10 & 1.425 & 0.276 & 6.03 & 0.218 \\
\hline & $4 x$ & 10 & 1.093 & 0.296 & 10 & 1.358 & 0.218 & 10.81 & 0.0524 \\
\hline & $6 x \_29$ & 4 & 0.789 & 0.248 & 3 & 1.004 & 0.147 & 11.99 & 0.629 \\
\hline & $6 x \_35$ & 4 & 0.856 & 0.214 & 9 & 0.869 & 0.111 & 0.75 & 0.71 \\
\hline \multirow[t]{4}{*}{$q_{E}$} & $2 x$ & 10 & 0.45 & 0.178 & 10 & 0.728 & 0.289 & 23.60 & 0.0147 \\
\hline & $4 x$ & 10 & 0.698 & 0.244 & 10 & 0.869 & 0.269 & 10.91 & 0.123 \\
\hline & $6 x \_29$ & 4 & 0.35 & 0.108 & 3 & 0.605 & 0.149 & 26.70 & 0.0571 \\
\hline & $6 x \_35$ & 4 & 0.801 & 0.225 & 9 & 0.889 & 0.18 & 5.21 & 0.414 \\
\hline \multirow[t]{4}{*}{$\bar{q}$} & $2 x$ & 10 & 0.172 & 0.057 & 10 & 0.178 & 0.057 & 1.71 & 0.912 \\
\hline & $4 x$ & 10 & 0.263 & 0.06 & 10 & 0.229 & 0.052 & -6.91 & 0.19 \\
\hline & $6 x \_29$ & 4 & 0.12 & 0.015 & 3 & 0.131 & 0.005 & 4.38 & 0.4 \\
\hline & $6 x \_35$ & 4 & 0.258 & 0.171 & 9 & 0.256 & 0.085 & -0.39 & 0.71 \\
\hline \multirow[t]{4}{*}{$\overline{N P Q_{E}}$} & $2 x$ & 10 & 0.512 & 0.198 & 10 & 0.838 & 0.33 & 24.15 & 0.0232 \\
\hline & $4 x$ & 10 & 0.871 & 0.33 & 10 & 1.044 & 0.349 & 9.03 & 0.315 \\
\hline & $6 x \_29$ & 4 & 0.375 & 0.109 & 3 & 0.662 & 0.167 & 27.68 & 0.0571 \\
\hline & $6 x \_35$ & 4 & 0.962 & 0.294 & 9 & 1.06 & 0.289 & 4.85 & 0.604 \\
\hline \multirow[t]{4}{*}{$\overline{N P Q_{1}}$} & $2 x$ & 10 & 0.213 & 0.092 & 10 & 0.222 & 0.093 & 2.07 & 0.912 \\
\hline & $4 x$ & 10 & 0.365 & 0.11 & 10 & 0.302 & 0.086 & -9.45 & 0.19 \\
\hline & $6 x \_29$ & 4 & 0.136 & 0.02 & 3 & 0.151 & 0.006 & 5.23 & 0.4 \\
\hline & $6 x \_35$ & 4 & 0.419 & 0.409 & 9 & 0.359 & 0.156 & -7.71 & 0.71 \\
\hline \multirow[t]{4}{*}{$q P$} & $2 x$ & 10 & 0.601 & 0.065 & 10 & 0.6 & 0.05 & -0.08 & 0.0433 \\
\hline & $4 x$ & 10 & 0.486 & 0.075 & 10 & 0.569 & 0.049 & 7.87 & 0.529 \\
\hline & $6 x \_29$ & 4 & 0.447 & 0.095 & 3 & 0.509 & 0.023 & 6.49 & 0.912 \\
\hline & $6 x \_35$ & 4 & 0.569 & 0.149 & 9 & 0.573 & 0.069 & 0.35 & 0.00893 \\
\hline \multirow[t]{4}{*}{$q N$} & $2 x$ & 10 & 0.481 & 0.117 & 10 & 0.593 & 0.101 & 10.43 & 0.629 \\
\hline & $4 x$ & 10 & 0.611 & 0.139 & 10 & 0.669 & 0.099 & 4.53 & 0.825 \\
\hline & $6 x \_29$ & 4 & 0.371 & 0.055 & 3 & 0.524 & 0.061 & 17.09 & 0.0571 \\
\hline & $6 x \_35$ & 4 & 0.776 & 0.268 & 9 & 0.802 & 0.153 & 1.65 & 0.604 \\
\hline
\end{tabular}




\begin{tabular}{|c|c|c|c|c|c|c|c|c|c|}
\hline \multirow{2}{*}{ Parameter } & \multirow{2}{*}{ Ploidy } & \multicolumn{3}{|c|}{ Control treatment (C) } & \multicolumn{3}{|c|}{ Stress treatment $(\mathrm{C})$} & \multirow{2}{*}{ S-C } & \multirow{2}{*}{$\mathbf{p}$} \\
\hline & & $\mathbf{n}$ & mean & sd & $\mathbf{n}$ & mean & sd & & \\
\hline \multirow[t]{4}{*}{$q L$} & $2 x$ & 10 & 0.299 & 0.097 & 10 & 0.317 & 0.057 & 2.92 & 0.353 \\
\hline & $4 x$ & 10 & 0.226 & 0.069 & 10 & 0.306 & 0.059 & 15.04 & 0.0147 \\
\hline & $6 x \_29$ & 4 & 0.16 & 0.057 & 3 & 0.23 & 0.023 & 17.95 & 0.0571 \\
\hline & $6 x \_35$ & 4 & 0.397 & 0.202 & 9 & 0.402 & 0.101 & 0.63 & 0.71 \\
\hline
\end{tabular}


Table S10. Pairwise comparison of inductive curve parameters in different treatments. Significant results are in bold.

\begin{tabular}{|c|c|c|c|c|c|c|c|c|}
\hline \multirow{2}{*}{ Parameter } & \multirow{2}{*}{ group1 } & \multirow{2}{*}{ group2 } & \multicolumn{3}{|c|}{ Control } & \multicolumn{3}{|c|}{ Stress } \\
\hline & & & statistic & $\mathbf{p}$ & p.adj & statistic & $p$ & p.adj \\
\hline \multirow[t]{6}{*}{ NPQ } & $2 x$ & $4 x$ & 13 & 0.00400 & 0.02300 & 31 & 0.16500 & 0.99000 \\
\hline & $2 x$ & $6 x \_29$ & 32 & 0.10600 & 0.63600 & 21 & 0.37100 & 1.00000 \\
\hline & $2 x$ & $6 x \_35$ & 5 & 0.03600 & 0.21600 & 27 & 0.15600 & 0.93600 \\
\hline & $4 x$ & $6 x \_29$ & 36 & 0.02400 & 0.14400 & 28 & 0.02800 & 0.16800 \\
\hline & $4 x$ & $6 x \_35$ & 18 & 0.83900 & 1.00000 & 44 & 0.96800 & 1.00000 \\
\hline & $6 x+29$ & $6 x \_35$ & 0 & 0.02900 & 0.17200 & 2 & 0.03600 & 0.21800 \\
\hline \multirow[t]{6}{*}{$P Q$} & $2 x$ & $4 x$ & 67 & 0.21800 & 1.00000 & 64 & 0.31500 & 1.00000 \\
\hline & $2 x$ & $6 x \_29$ & 40 & 0.00200 & 0.01200 & 27 & 0.04900 & 0.29400 \\
\hline & $2 x$ & $6 x \_35$ & 38 & 0.00800 & 0.04800 & 84 & 0.00065 & 0.00400 \\
\hline & $4 x$ & $6 x \_29$ & 35 & 0.03600 & 0.21600 & 27 & 0.04900 & 0.29400 \\
\hline & $4 x$ & 6x_35 & 29 & 0.24000 & 1.00000 & 86 & 0.00026 & 0.00200 \\
\hline & $6 x+29$ & $6 x \_35$ & 5 & 0.48600 & 1.00000 & 20 & 0.28200 & 1.00000 \\
\hline \multirow[t]{6}{*}{$q_{E}$} & $2 x$ & $4 x$ & 18 & 0.01500 & 0.08800 & 29 & 0.12300 & 0.73800 \\
\hline & $2 x$ & $6 x \_29$ & 27 & 0.37400 & 1.00000 & 17 & 0.81100 & 1.00000 \\
\hline & $2 x$ & 6x_35 & 3 & 0.01400 & 0.08400 & 25 & 0.11300 & 0.67800 \\
\hline & $4 x$ & $6 x \_29$ & 36 & 0.02400 & 0.14400 & 25 & 0.11200 & 0.67200 \\
\hline & $4 x$ & $6 x \_35$ & 15 & 0.53900 & 1.00000 & 35 & 0.44700 & 1.00000 \\
\hline & $6 x \_29$ & $6 x \_35$ & 0 & 0.02900 & 0.17200 & 2 & 0.03600 & 0.21800 \\
\hline \multirow[t]{6}{*}{$q_{l}$} & $2 x$ & $4 x$ & 14 & 0.00500 & 0.03100 & 22 & 0.03500 & 0.21300 \\
\hline & $2 x$ & $6 x \_29$ & 34 & 0.05400 & 0.32300 & 24 & 0.16100 & 0.96600 \\
\hline & $2 x$ & 6x_35 & 12 & 0.30400 & 1.00000 & 21 & 0.05400 & 0.32100 \\
\hline & $4 x$ & $6 x \_29$ & 40 & 0.00200 & 0.01200 & 30 & 0.00700 & 0.04200 \\
\hline & $4 x$ & $6 x \_35$ & 26 & 0.45400 & 1.00000 & 37 & 0.54900 & 1.00000 \\
\hline & $6 x \_29$ & $6 x \_35$ & 2 & 0.11400 & 0.68400 & 2 & 0.03600 & 0.21800 \\
\hline \multirow[t]{6}{*}{$N P Q_{E}$} & $2 x$ & $4 x$ & 13 & 0.00400 & 0.02300 & 30 & 0.14300 & 0.85800 \\
\hline & $2 x$ & $6 x \_29$ & 28 & 0.30400 & 1.00000 & 20 & 0.46900 & 1.00000 \\
\hline & $2 x$ & $6 x \_35$ & 5 & 0.03600 & 0.21600 & 23 & 0.07900 & 0.47300 \\
\hline & $4 x$ & $6 x \_29$ & 36 & 0.02400 & 0.14400 & 28 & 0.02800 & 0.16800 \\
\hline & $4 x$ & 6x_35 & 16 & 0.63500 & 1.00000 & 39 & 0.66100 & 1.00000 \\
\hline & $6 x+29$ & $6 x \_35$ & 0 & 0.02900 & 0.17200 & 2 & 0.03600 & 0.21800 \\
\hline \multirow[t]{6}{*}{$N P Q_{1}$} & $2 x$ & $4 x$ & 14 & 0.00500 & 0.03100 & 22 & 0.03500 & 0.21300 \\
\hline & $2 x$ & $6 x \_29$ & 34 & 0.05400 & 0.32300 & 24 & 0.16100 & 0.96600 \\
\hline & $2 x$ & $6 x \_35$ & 12 & 0.30400 & 1.00000 & 21 & 0.05400 & 0.32100 \\
\hline & $4 x$ & $6 x \_29$ & 40 & 0.00200 & 0.01200 & 30 & 0.00700 & 0.04200 \\
\hline & $4 x$ & 6x_35 & 26 & 0.45400 & 1.00000 & 37 & 0.54900 & 1.00000 \\
\hline & $6 x \_29$ & $6 x \_35$ & 2 & 0.11400 & 0.68400 & 2 & 0.03600 & 0.21800 \\
\hline \multirow[t]{5}{*}{$q P$} & $2 x$ & $4 x$ & 88 & 0.00300 & 0.01700 & 66 & 0.24700 & 1.00000 \\
\hline & $2 x$ & $6 x \_29$ & 40 & 0.00200 & 0.01200 & 27 & 0.04900 & 0.29400 \\
\hline & $2 x$ & $6 x \_35$ & 21 & 0.94500 & 1.00000 & 54 & 0.49700 & 1.00000 \\
\hline & $4 x$ & $6 x \_29$ & 24 & 0.63500 & 1.00000 & 26 & 0.07700 & 0.46100 \\
\hline & $4 x$ & $6 x \_35$ & 9 & 0.14200 & 0.85200 & 38 & 0.60400 & 1.00000 \\
\hline
\end{tabular}




\begin{tabular}{|c|c|c|c|c|c|c|c|c|}
\hline \multirow{2}{*}{ Parameter } & \multirow{2}{*}{ group1 } & \multirow{2}{*}{ group2 } & \multicolumn{3}{|c|}{ Control } & \multicolumn{3}{|c|}{ Stress } \\
\hline & & & statistic & $\mathbf{p}$ & p.adj & statistic & $p$ & p.adj \\
\hline \multirow[t]{6}{*}{$q N$} & $2 x$ & $4 x$ & 21 & 0.02900 & 0.17300 & 33 & 0.21800 & 1.00000 \\
\hline & $2 x$ & $6 x \_29$ & 31 & 0.14200 & 0.85200 & 22 & 0.28700 & 1.00000 \\
\hline & $2 x$ & $6 x \_35$ & 5 & 0.03600 & 0.21600 & 11 & 0.00400 & 0.02500 \\
\hline & $4 x$ & $6 x \_29$ & 36 & 0.02400 & 0.14400 & 28 & 0.02800 & 0.16800 \\
\hline & $4 x$ & $6 x \_35$ & 10 & 0.18800 & 1.00000 & 24 & 0.09500 & 0.56800 \\
\hline & $6 x \_29$ & $6 \times \_35$ & 0 & 0.02900 & 0.17200 & 1 & 0.01800 & 0.10900 \\
\hline \multirow[t]{6}{*}{$q L$} & $2 x$ & $4 x$ & 68 & 0.19000 & 1.00000 & 60 & 0.48100 & 1.00000 \\
\hline & $2 x$ & $6 x \_29$ & 35 & 0.03600 & 0.21600 & 28 & 0.02800 & 0.16800 \\
\hline & $2 x$ & $6 x \_35$ & 12 & 0.30400 & 1.00000 & 22 & 0.06500 & 0.39200 \\
\hline & $4 x$ & $6 x \_29$ & 32 & 0.10600 & 0.63600 & 27 & 0.04900 & 0.29400 \\
\hline & $4 x$ & $6 x \_35$ & 9 & 0.14200 & 0.85200 & 22 & 0.06500 & 0.39200 \\
\hline & $6 x+29$ & $6 \times \_35$ & 3 & 0.20000 & 1.00000 & 2 & 0.03600 & 0.21800 \\
\hline
\end{tabular}


Table S11. Summary statistics and $P$ - values of Wilcoxon-Mann-Whitney-Test for determination of effects of prolonged photoperiod on JIP-test parameters between treatments. Summary statistic of treatment. S-C: proportion of alternation of the mean value, calculated as (Stress - Control/(Stress + Control)*100. Significant results are in bold.

\begin{tabular}{|c|c|c|c|c|c|c|c|c|c|}
\hline \multirow{2}{*}{ Parameter } & \multirow{2}{*}{ Group } & \multicolumn{3}{|c|}{ Control treatment } & \multicolumn{3}{|c|}{ Stress treatment } & \multirow{2}{*}{ S-C } & \multirow{2}{*}{ p } \\
\hline & & $\mathbf{n}$ & mean & sd & $\mathrm{n}$ & mean & sd & & \\
\hline \multirow[t]{4}{*}{$\mathrm{V}_{\mathrm{i}}$} & $2 x$ & 10 & 0.715 & 0.031 & 10 & 0.764 & 0.021 & 3.313 & 0.00105 \\
\hline & $4 \mathrm{x}$ & 10 & 0.792 & 0.025 & 11 & 0.792 & 0.045 & 0.000 & 0.80500 \\
\hline & $6 x \_29$ & 4 & 0.748 & 0.018 & 3 & 0.759 & 0.041 & 0.730 & 1.00000 \\
\hline & $6 x \_35$ & 7 & 0.741 & 0.077 & 7 & 0.687 & 0.11 & -3.782 & 0.31800 \\
\hline \multirow[t]{4}{*}{$\mathrm{V}_{\mathrm{j}}$} & $2 x$ & 10 & 0.382 & 0.033 & 10 & 0.41 & 0.036 & 3.535 & 0.12300 \\
\hline & $4 x$ & 10 & 0.396 & 0.051 & 11 & 0.412 & 0.085 & 1.980 & 0.83300 \\
\hline & $6 x \_29$ & 4 & 0.418 & 0.036 & 3 & 0.394 & 0.043 & -2.956 & 0.62900 \\
\hline & $6 x \_35$ & 7 & 0.462 & 0.053 & 7 & 0.447 & 0.057 & -1.650 & 0.62000 \\
\hline \multirow[t]{4}{*}{$\mathrm{F}_{\mathrm{M}} / \mathrm{F}_{0}$} & $2 x$ & 10 & 5.71 & 0.425 & 10 & 5.228 & 0.326 & -4.407 & 0.00893 \\
\hline & $4 x$ & 10 & 5.602 & 0.295 & 11 & 5.448 & 0.587 & -1.394 & 0.65400 \\
\hline & $6 x \_29$ & 4 & 5.656 & 0.188 & 3 & 5.597 & 0.497 & -0.524 & 0.85700 \\
\hline & $6 x \_35$ & 7 & 3.848 & 1.03 & 7 & 2.991 & 1.051 & -12.531 & 0.12800 \\
\hline \multirow[t]{4}{*}{$\mathrm{Fv} / \mathrm{F}_{0}$} & $2 x$ & 10 & 4.71 & 0.425 & 10 & 4.228 & 0.326 & -5.393 & 0.00893 \\
\hline & $4 x$ & 10 & 4.602 & 0.295 & 11 & 4.448 & 0.587 & -1.702 & 0.65400 \\
\hline & $6 x \_29$ & 4 & 4.656 & 0.188 & 3 & 4.597 & 0.497 & -0.638 & 0.85700 \\
\hline & $6 x \_35$ & 7 & 2.848 & 1.03 & 7 & 1.991 & 1.051 & -17.710 & 0.12800 \\
\hline \multirow[t]{4}{*}{$\mathrm{Fv} / \mathrm{F}_{\mathrm{M}}$} & $2 x$ & 10 & 0.824 & 0.013 & 10 & 0.808 & 0.012 & -0.980 & 0.01260 \\
\hline & $4 x$ & 10 & 0.821 & 0.009 & 11 & 0.815 & 0.021 & -0.367 & 0.67200 \\
\hline & $6 x \_29$ & 4 & 0.823 & 0.006 & 3 & 0.82 & 0.015 & -0.183 & 0.85700 \\
\hline & $6 x \_35$ & 7 & 0.724 & 0.072 & 7 & 0.633 & 0.111 & -6.706 & 0.11000 \\
\hline \multirow[t]{4}{*}{$\mathrm{M}_{0}$} & $2 x$ & 10 & 0.601 & 0.092 & 10 & 0.695 & 0.095 & 7.253 & 0.03550 \\
\hline & $4 x$ & 10 & 0.65 & 0.125 & 11 & 0.643 & 0.205 & -0.541 & 0.86000 \\
\hline & $6 x \_29$ & 4 & 0.694 & 0.072 & 3 & 0.63 & 0.151 & -4.834 & 0.85700 \\
\hline & $6 x \_35$ & 7 & 0.935 & 0.225 & 7 & 1.043 & 0.229 & 5.460 & 0.45600 \\
\hline \multirow[t]{4}{*}{ Area } & $2 x$ & 10 & 11413795 & 1795993 & 10 & 10500268 & 1472647 & -4.169 & 0.39300 \\
\hline & $4 x$ & 10 & 9861305 & 1895808 & 11 & 7946877 & 3442061 & -10.750 & 0.38700 \\
\hline & $6 x \_29$ & 4 & 10785254 & 2097474 & 3 & 11355959 & 590010.4 & 2.578 & 0.40000 \\
\hline & $6 x \_35$ & 7 & 15959414 & 8015471 & 7 & 24223637 & 14561164 & 20.566 & 0.80500 \\
\hline \multirow[t]{4}{*}{ Fix.Area } & $2 x$ & 10 & 27204510 & 2434725 & 10 & 25168040 & 3171928 & -3.888 & 0.28000 \\
\hline & $4 x$ & 10 & 30394650 & 2801993 & 11 & 22873332 & 9660293 & -14.120 & 0.00479 \\
\hline & $6 x \_29$ & 4 & 26113779 & 3276642 & 3 & 26233125 & 1841156 & 0.228 & 0.62900 \\
\hline & $6 x \_35$ & 7 & 30091663 & 3752652 & 7 & 22856067 & 7499010 & -13.666 & 0.05300 \\
\hline \multirow[t]{4}{*}{$\mathrm{Sm}$} & $2 x$ & 10 & 499.066 & 100.227 & 10 & 504.429 & 50.166 & 0.534 & 0.57900 \\
\hline & $4 x$ & 10 & 391.935 & 96.541 & 11 & 385.584 & 109.357 & -0.817 & 0.65400 \\
\hline & $6 x \_29$ & 4 & 488.675 & 65.249 & 3 & 515.18 & 12.911 & 2.640 & 0.40000 \\
\hline & $6 x \_35$ & 7 & 753.133 & 511.619 & 7 & 2034.586 & 1436.457 & 45.968 & 0.25900 \\
\hline \multirow[t]{4}{*}{ Ss } & $2 x$ & 10 & 0.643 & 0.052 & 10 & 0.594 & 0.035 & -3.961 & 0.01720 \\
\hline & $4 x$ & 10 & 0.616 & 0.042 & 11 & 0.665 & 0.093 & 3.825 & 0.17300 \\
\hline & $6 x \_29$ & 4 & 0.604 & 0.028 & 3 & 0.641 & 0.094 & 2.972 & 0.85700 \\
\hline & $6 x \_35$ & 7 & 0.508 & 0.079 & 7 & 0.444 & 0.096 & -6.723 & 0.25900 \\
\hline
\end{tabular}




\begin{tabular}{|c|c|c|c|c|c|c|c|c|c|}
\hline \multirow{2}{*}{ Parameter } & \multirow{2}{*}{ Group } & \multicolumn{3}{|c|}{ Control treatment } & \multicolumn{3}{|c|}{ Stress treatment } & \multirow{2}{*}{ S-C } & \multirow{2}{*}{$\mathbf{p}$} \\
\hline & & $\mathbf{n}$ & mean & sd & $\mathbf{n}$ & mean & sd & & \\
\hline \multirow[t]{4}{*}{$N$} & $2 x$ & 10 & 777.587 & 151.779 & 10 & 850.901 & 98.376 & 4.502 & 0.07530 \\
\hline & $4 x$ & 10 & 634.705 & 138.84 & 11 & 593.303 & 170.98 & -3.371 & 0.91800 \\
\hline & $6 x \_29$ & 4 & 810.54 & 112.543 & 3 & 813.34 & 99.779 & 0.172 & 1.00000 \\
\hline & $6 x \_35$ & 7 & 1585.056 & 1294.919 & 7 & 5217.227 & 4045.185 & 53.396 & 0.31800 \\
\hline \multirow[t]{4}{*}{ Phi_Po } & $2 x$ & 10 & 0.824 & 0.013 & 10 & 0.808 & 0.012 & -0.980 & 0.01260 \\
\hline & $4 x$ & 10 & 0.821 & 0.009 & 11 & 0.815 & 0.021 & -0.367 & 0.67200 \\
\hline & $6 x \_29$ & 4 & 0.823 & 0.006 & 3 & 0.82 & 0.015 & -0.183 & 0.85700 \\
\hline & $6 x \_35$ & 7 & 0.724 & 0.072 & 7 & 0.633 & 0.111 & -6.706 & 0.11000 \\
\hline \multirow[t]{4}{*}{ Psi_0 } & $2 x$ & 10 & 0.617 & 0.033 & 10 & 0.59 & 0.036 & -2.237 & 0.12300 \\
\hline & $4 x$ & 10 & 0.604 & 0.051 & 11 & 0.588 & 0.085 & -1.342 & 0.83300 \\
\hline & $6 x \_29$ & 4 & 0.582 & 0.036 & 3 & 0.606 & 0.043 & 2.020 & 0.62900 \\
\hline & $6 x \_35$ & 7 & 0.538 & 0.053 & 7 & 0.553 & 0.057 & 1.375 & 0.62000 \\
\hline \multirow[t]{4}{*}{ Phi_E 0} & $2 x$ & 10 & 0.509 & 0.029 & 10 & 0.477 & 0.034 & -3.245 & 0.02560 \\
\hline & $4 x$ & 10 & 0.496 & 0.044 & 11 & 0.48 & 0.08 & -1.639 & 0.69800 \\
\hline & $6 x \_29$ & 4 & 0.479 & 0.032 & 3 & 0.497 & 0.042 & 1.844 & 0.62900 \\
\hline & $6 x+35$ & 7 & 0.392 & 0.068 & 7 & 0.348 & 0.056 & -5.946 & 0.25000 \\
\hline \multirow[t]{4}{*}{ Phi_Pav } & $2 x$ & 10 & 930.326 & 9.949 & 10 & 939.887 & 4.589 & 0.511 & 0.01850 \\
\hline & $4 x$ & 10 & 937.974 & 8.581 & 11 & 928.619 & 32.596 & -0.501 & 0.91800 \\
\hline & $6 x \_29$ & 4 & 936.878 & 9.82 & 3 & 943.21 & 6.593 & 0.337 & 0.40000 \\
\hline & $6 x \_35$ & 7 & 940 & 8.907 & 7 & 948.514 & 12.36 & 0.451 & 0.20900 \\
\hline \multirow[t]{4}{*}{ Phi_Do } & $2 x$ & 10 & 0.176 & 0.013 & 10 & 0.192 & 0.012 & 4.348 & 0.01260 \\
\hline & $4 x$ & 10 & 0.179 & 0.009 & 11 & 0.185 & 0.021 & 1.648 & 0.67200 \\
\hline & $6 x \_29$ & 4 & 0.177 & 0.006 & 3 & 0.18 & 0.015 & 0.840 & 0.85700 \\
\hline & $6 x \_35$ & 7 & 0.276 & 0.072 & 7 & 0.367 & 0.111 & 14.152 & 0.11000 \\
\hline \multirow[t]{4}{*}{$\mathrm{ABS} / \mathrm{RC}$} & $2 x$ & 10 & 1.902 & 0.181 & 10 & 2.09 & 0.154 & 4.709 & 0.01400 \\
\hline & $4 x$ & 10 & 1.987 & 0.162 & 11 & 1.885 & 0.29 & -2.634 & 0.38700 \\
\hline & 6x_29 & 4 & 2.017 & 0.099 & 3 & 1.931 & 0.295 & -2.178 & 0.85700 \\
\hline & $6 \times 35$ & 7 & 2.714 & 0.756 & 7 & 3.866 & 1.399 & 17.508 & 0.10500 \\
\hline \multirow[t]{4}{*}{$\mathrm{Dl} / \mathrm{RC}$} & $2 x$ & 10 & 0.336 & 0.052 & 10 & 0.403 & 0.054 & 9.066 & 0.01150 \\
\hline & $4 x$ & 10 & 0.356 & 0.045 & 11 & 0.354 & 0.087 & -0.282 & 0.86300 \\
\hline & $6 x \_29$ & 4 & 0.357 & 0.028 & 3 & 0.35 & 0.078 & -0.990 & 0.62900 \\
\hline & $6 x \_35$ & 7 & 0.867 & 0.551 & 7 & 1.545 & 0.905 & 28.109 & 0.12800 \\
\hline \multirow[t]{4}{*}{$\mathrm{TR} / \mathrm{RC}$} & $2 x$ & 10 & 1.566 & 0.134 & 10 & 1.688 & 0.102 & 3.749 & 0.01470 \\
\hline & $4 x$ & 10 & 1.631 & 0.12 & 11 & 1.572 & 0.251 & -1.842 & 0.42600 \\
\hline & $6 x \_29$ & 4 & 1.659 & 0.073 & 3 & 1.582 & 0.219 & -2.376 & 0.85700 \\
\hline & $6 x \_35$ & 7 & 2 & 0 & 7 & 2.393 & 0.434 & 8.946 & 0.00372 \\
\hline \multirow[t]{4}{*}{$\mathrm{ET} / \mathrm{RC}$} & $2 x$ & 10 & 0.964 & 0.067 & 10 & 0.992 & 0.047 & 1.431 & 0.21200 \\
\hline & $4 x$ & 10 & 0.981 & 0.057 & 11 & 0.886 & 0.081 & -5.088 & 0.00365 \\
\hline & $6 x \_29$ & 4 & 0.965 & 0.071 & 3 & 0.952 & 0.079 & -0.678 & 1.00000 \\
\hline & $6 \times 35$ & 7 & 0.987 & 0.023 & 7 & 1.301 & 0.331 & 13.724 & 0.01870 \\
\hline \multirow[t]{4}{*}{ Pi_Abs } & $2 x$ & 10 & 4.121 & 1.014 & 10 & 3.005 & 0.786 & -15.661 & 0.02880 \\
\hline & $4 x$ & 10 & 3.696 & 1.044 & 11 & 3.99 & 2.502 & 3.825 & 1.00000 \\
\hline & $6 x \_29$ & 4 & 3.267 & 0.65 & 3 & 3.909 & 1.715 & 8.946 & 0.85700 \\
\hline & $6 \times 35$ & 7 & 1.41 & 0.949 & 7 & 0.787 & 0.645 & -28.357 & 0.12400 \\
\hline
\end{tabular}


Table S12. Pairwise comparison of JIP-test parameters in different treatments. Significant results are in bold.

\begin{tabular}{|c|c|c|c|c|c|c|c|c|}
\hline \multirow{2}{*}{ Parameter } & \multirow{2}{*}{ group1 } & \multirow{2}{*}{ group2 } & \multicolumn{3}{|r|}{ Control } & \multicolumn{3}{|r|}{ Stress } \\
\hline & & & statistic & $p$ & p.adj & statistic & $p$ & p.adj \\
\hline \multirow[t]{6}{*}{$\mathrm{ABS} / \mathrm{RC}$} & $2 x$ & $4 x$ & 36 & 0.31500 & 1.00000 & 81 & 0.07200 & 0.26700 \\
\hline & $2 x$ & $6 x \_29$ & 12 & 0.30400 & 1.00000 & 18 & 0.67200 & 1.00000 \\
\hline & $2 x$ & 6x_35 & 6 & 0.00500 & 0.03100 & 6 & 0.00500 & 0.02700 \\
\hline & $4 \mathrm{x}$ & $6 x \_29$ & 14 & 0.45400 & 1.00000 & 15 & 0.88500 & 1.00000 \\
\hline & $4 x$ & 6x_35 & 12 & 0.02700 & 0.13600 & 3.5 & 0.00200 & 0.01100 \\
\hline & $6 x \_29$ & $6 x \_35$ & 9 & 0.38600 & 1.00000 & 2 & 0.06700 & 0.26700 \\
\hline \multirow[t]{6}{*}{$\mathrm{DI} / \mathrm{RC}$} & $2 x$ & $4 x$ & 39.5 & 0.45000 & 1.00000 & 76 & 0.15200 & 0.45600 \\
\hline & $2 x$ & $6 x \_29$ & 15 & 0.53900 & 1.00000 & 20 & 0.46900 & 0.93800 \\
\hline & $2 x$ & $6 x \_35$ & 3 & 0.00072 & 0.00400 & 0 & 0.00010 & 0.00052 \\
\hline & $4 x$ & $6 x \_29$ & 16 & 0.63500 & 1.00000 & 15 & 0.88500 & 0.93800 \\
\hline & $4 x$ & $6 x \_35$ & 5 & 0.00200 & 0.01000 & 0 & 0.00006 & 0.00038 \\
\hline & $6 x \_29$ & $6 x \_35$ & 3 & 0.04200 & 0.17000 & 0 & 0.01700 & 0.06700 \\
\hline \multirow[t]{6}{*}{$\mathrm{TR}_{0} / \mathrm{RC}$} & $2 x$ & $4 x$ & 33 & 0.21800 & 0.65400 & 77 & 0.13200 & 0.39600 \\
\hline & $2 x$ & $6 x \_29$ & 12 & 0.30400 & 0.65400 & 17 & 0.81100 & 1.00000 \\
\hline & $2 x$ & 6x_35 & 0 & 0.00049 & 0.00300 & 0 & 0.00010 & 0.00062 \\
\hline & $4 x$ & $6 x \_29$ & 14 & 0.45400 & 0.65400 & 16 & 1.00000 & 1.00000 \\
\hline & $4 x$ & $6 x \_35$ & 0 & 0.00049 & 0.00300 & 0.5 & 0.00068 & 0.00300 \\
\hline & $6 x+29$ & $6 x \_35$ & 0 & 0.00300 & 0.01300 & 0 & 0.01700 & 0.06700 \\
\hline \multirow[t]{6}{*}{$\mathrm{ET}_{0} / \mathrm{RC}$} & $2 x$ & $4 x$ & 41.5 & 0.54500 & 1.00000 & 99 & 0.00200 & 0.01100 \\
\hline & $2 x$ & $6 x \_29$ & 22 & 0.83900 & 1.00000 & 19 & 0.55400 & 0.62200 \\
\hline & $2 x$ & $6 x \_35$ & 22 & 0.21700 & 1.00000 & 13.5 & 0.04000 & 0.16100 \\
\hline & $4 x$ & $6 x \_29$ & 23 & 0.73300 & 1.00000 & 9.5 & 0.31100 & 0.62200 \\
\hline & $4 x$ & $6 x \_35$ & 32 & 0.80500 & 1.00000 & 3 & 0.00044 & 0.00300 \\
\hline & $6 x+29$ & $6 x \_35$ & 8 & 0.27600 & 1.00000 & 3 & 0.11700 & 0.35100 \\
\hline \multirow[t]{6}{*}{ Pi_Abs } & $2 x$ & $4 x$ & 59 & 0.52900 & 1.00000 & 44 & 0.46800 & 1.00000 \\
\hline & $2 x$ & $6 x \_29$ & 29 & 0.24000 & 0.72000 & 11 & 0.57300 & 1.00000 \\
\hline & $2 x$ & $6 x \_35$ & 68 & 0.00200 & 0.00900 & 69 & 0.00021 & 0.00100 \\
\hline & $4 x$ & $6 x \_29$ & 25 & 0.53900 & 1.00000 & 16 & 1.00000 & 1.00000 \\
\hline & $4 x$ & $6 x \_35$ & 68 & 0.00200 & 0.00900 & 73 & 0.00075 & 0.00400 \\
\hline & $6 x \_29$ & $6 x \_35$ & 26 & 0.02900 & 0.11800 & 21 & 0.01700 & 0.06700 \\
\hline
\end{tabular}




\section{Chapter 4: Discussion}

In this thesis, I had obtained better insights into the role of polyploidy in the complex environmental stress of the photoperiod alternation that contributed to the physiological process of photosynthesis, hence concomitant to reproductive mode. Even though the present study is not complete in any way to deal with the various issues of plant evolution related to polyploidy, the model plants of facultative apomictic $R$. auricomus complex with three cytotype levels (diploid, tetraploid, and hexaploid) presented the role of polyploidy in buffering light stress. Results indicated a higher stress sensitivity of diploids as observed in ovule development and photosynthetics organ but a lesser stress effect in tetraploid and a stronger stress-tolerance in hexaploids. The establishment of apomixis seed development is complex. It involves three components: apomeiosis (formation of unreduced embryo sac), parthenogenesis (embryo development without fertilization of egg cell), and functional endosperm development (Nogler, 1984a; Kaushal et al., 2018). The photoperiod stress only affected the apomeiosis but not the later stages.

\subsection{Mode of ovule formation}

The comprehensive investigation of ovule development with cytological analysis of a total of 6505 ovules from the three cytotypes $R$. auricomus complex has shown the typical Polygonum type embryo sac and at a similar timing. In the sexual ovule, during megasporogenesis, the meiotic division of a megaspore mother cell produced four cells (megaspore tetrad), in which only one remains to develop into a functional megaspore, while the other three aborted. At megagametogenesis, the functional megaspore enlarged with the presence of vacuoles and continued with three times of nuclear division, producing a total of eight nuclei of embryo sac structure. On the other hand, in the asexual ovule, at megasporogenesis, one or more aposporous initial cell/s developed from an enlarged somatic cell near to functional megaspore. The aposporous cell grows faster than the megaspore and takes over the embryo sac development (Hojsgaard et al., 2014a). Under microscopic analysis, the hexaploids ovule presented a bigger cell size compared to tetraploids, while diploids presented the smaller size of the structure. Having a bigger cell size confers at least three consequences, i.e., first, increasing volume decreases surface area, which could impact cell signaling on limiting oxidative damage; second, impact on mechanical properties; and third, pertaining to the size of intracellular structure, e.g., spindle which contributes to cell division (Schoenfelder and Fox, 2015). The alternation of spindle structure in polyploid yeast leads to the increase of errors of chromosome segregation during the cell division process (Storchová et al., 2006). In reproductive tissues, polyploids release apomeiosis as a strategy to circumvent the unbalanced chromosomal segregation (Hojsgaard, 2018). Another study on disoriented 
chromosomes during microsporogenesis in diploid and allopolyploid $R$. auricomus among cytotype is presented in (Barke et al., 2020). Further study on megasporogenesis will provide a clearer understanding of factors triggering apomixis.

The cytological analysis also discriminated the alternation of the proportions of sexual and asexual ovule between the treatments and showed the sign of buffering stress in the establishment of apomixis in polyploids. The prolonged photoperiod might cause the accumulation of reactive oxygen species (ROS) in the flower buds, which trigger oxidative stress (Klatt et al., 2016; Abuelsoud et al., 2020) e.g., as indicated by the accumulation of lipid peroxidation (Nitschke et al., 2016). In facultative apomixis, apomeiosis and meiotic cell division are regulated by metabolites that are related to ROS scavenging (Mateo de Arias et al., 2020). In diploids of $R$. auricomus, the $16.5 \mathrm{~h}$ photoperiod was categorized as a mild abiotic stressor that mobilized the meiotic DNA repair system in the megaspore mother cell and triggered meiosis and megasporogenesis (Hörandl and Hadacek, 2013). The proportion of sexual ovules was $80.4 \%$ or c.a. $19.6 \%$ apospory were observed in diploids synthetic hybrid, and this related to the low frequency of natural diploid apomixis. A recent flow cytometric seed screening study reported the frequency of $1 \%$ of diploid apomictic seed in $R$. auricomus from temperate Europe (Karbstein et al., 2021).

On the other hand, in tetraploids and hexaploids, probably due to the better ROS scavenging (Deng et al., 2012; Wei et al., 2019), the oxidative stress from the same photoperiod treatment might be less strong to induce the DNA double strand breaks (Hörandl and Hadacek, 2013), and consequently meiosis and megasporogenesis might be disturbed and released apomeiosis that occupies embryo sac development. An example study on allopolyploids of Glycine dolichocarpa addressed the better light stress-tolerance compared to the diploid relatives indicated by the alternation of non-photochemical quenching followed by ROS scavenging (Coate et al., 2013). This result is correlated to our finding that polyploids regulated a stress buffer on NPQ parameters (Chapter 2). Nevertheless, metabolites analysis addressing the ROS scavenging among cytotypes will be immensely supporting our assumption.

The advantages of gene redundancy (more DNA repair templates) of polyploids protect the DNA from stress (Schoenfelder and Fox, 2015); hence a higher dosage of stress would be required to break the DNA as a consequence of buffering stress in polyploids (Hörandl and Hadacek, 2013). Polyploidy, on the other hand, challenges proper gene regulation by epigenetic disability due to the interaction between parental genomes (Comai, 2005). The high variation of the proportions of sexual ovules among individual of polyploids compared to diploids follow the assumption of the epigenetic mechanism as the background of phenotypic 
expression (Grimanelli, 2012; Schmidt et al., 2014). A similar pattern was reported from the same genus in Ranunculus kuepferi (Schinkel et al., 2020). Overall, the result of cytological analysis of ovule development supports the hypothesis of ploidy dependent-effects on the phenotypic feature of apomixis in angiosperms (Delgado et al., 2016; Kaushal et al., 2018) and under epigenetic regulation (Rodrigo et al., 2017).

\subsection{Mode of seed formation}

Mode of seed formation was analyzed with flow cytometry seed screening (FCSS) to a total of 14068 viable seeds from 83 individuals among three cytotypes growing in control and stress photoperiod. Hexaploids developed a bigger seed size, but diploids produced a higher quantity of seed set compared to polyploids. Nevertheless, high abortion rate of seeds compared to the rate of ovule formation was observed in all cytotypes and was still within the range (30 $60 \%$ ) of similar studies in R. auricomus (Izmaiłow, 1996; Hörandl, 2008; Hörandl and Temsch, 2009; Klatt et al., 2016; Barke et al., 2018). The seed quantity is also related to the pollen quality. In Apomictic seed, the embryo development was independent of the male genome (parthenogenesis). But for endosperm development, the contribution of male gamete is essential (Pseuodgamy) (Nogler, 1984a). The better quality of pollen of diploids and hexaploids was followed by a higher seed set, while in tetraploids, the lower pollen quality was also followed by a lower seed set. However, a high variation of pollen quality was reported in apomictic plants (Asker and Jerling, 1992). Our result revealed a neutral effect of photoperiod on the pollen quality and concomitant with the seed quality.

The formation of the viable seeds of $R$. auricomus requires the contribution of male gamete to fertilize the central nuclei for the development of endosperm. The FCSS results revealed that a low number of autonomous (poolen-independent) seeds (less than 10 seed per cytotype) were formed in all cytotypes, and that means almost all $R$. auricomus's seeds developed pseudogamously. The lower number of apomictic seeds in diploids was related to their sensitivity to the genomic imprinting; a constant ratio 2:1 for maternal $(m)$ and paternal $(p)$ genome contributed to the endosperm (Spielman et al., 2003; Vinkenoog et al., 2003). This imprinting factor contributed to the reduction of the number of putative apomictic seeds in diploids growing under control treatment. In contrast, polyploids presented a lower number of sexual seeds but a higher number of apomictic pseudogamous seeds, and a conspicuous number of $B_{I I I}$ hybrids in tetraploids. The formation of pseudogamous endosperm is predominant in apomictic plants (Mogie, 1992). The relaxation of genomic imprinting in polyploids was suggested due to the epigenetic mutation during endosperm development (Kaushal et al., 2018) and addressing the better seed set of hexaploids compared to tetraploid as observed in this study and another study of Potentilla puberula (Dobeš et al., 2018). The 
presence of $B_{\| I}$ hybrids was suggested either by the longer receptivity of egg cell in tetraploids as reported in the synthetic hybrid of $R$. auricomus (Barke et al., 2018) or by the hybridity contribution on the establishment of apomixis (Schinkel, 2019).

The peak index (PI) of FCSS indicated the DNA content in embryo and endosperm and represented the mode of seed development (Klatt et al., 2016; Schinkel et al., 2016; Barke et al., 2018). In the sexual seed of diploids, the reduced egg cell $(1 \mathrm{~m})$ is fertilized by a reduced sperm (1p) producing embryo with a set of the chromosome $[1(m)+1(p)]$, whereas in endosperm, the two reduced polar nuclei $(1 \mathrm{~m}+1 \mathrm{~m})$ are fertilized by a reduced sperm cell $(1 \mathrm{p})$ producing endosperm $[1(m)+1(m)+1(p)]$. Within these set chromosomes in a seed, the PI value 1.5 indicated that the seed developed sexually. While in apomictic seed, PI value is 2.0, 2.5, 3.0 depending on the contribution of pollen nuclei to endosperm. The FCSS revealed that photoperiod treatments did not alter the development of the seeds of $R$. auricomus. The seeds of diploid plants generally developed sexually with a lower amount of apomictic seeds (3 seeds in total). In contrast, polyploids developed their seeds asexually (>90\% seeds). The lower sexual seed formation in polyploids indicated a higher aborting rate of meiotic megaspores and replacement by apomeiosis that completed the megagametogenesis. The development of sexual ovules was less competitive compared to the development of aposporous embryo sac (AES) during seed development (Hojsgaard et al., 2013; Klatt et al., 2016; Hodač et al., 2019). In aposporous embryo sac, the unreduced egg cell develops parthenogenetically (without fertilization with sperm nucleus) into an embryo, while the unreduced polar nuclei develop pseudogamously (fertilized by a sperm cell/s) into endosperm. The dominance of apomictic seed formation in polyploids in this study is in line with the general observation that apomixis mostly occurs in polyploid plants with the presence of a lower number in diploids, supporting the hypothesis that polyploidy facilitates the establishment of apomixis (Hojsgaard and Hörandl, 2019).

\subsection{Photoprotective mechanisms}

Alternation on the mode of ovule formation was influenced by mid stress effect from prolonged photoperiod. The presence of light stress effects in $R$. auricomus was observed by measurement of photosynthesis performance with Chlorophyll a fluorescent as a proxy of photoprotection mechanisms. Measurements were conducted in the first basal leaf that appeared with flower buds. The plant generates mechanism to avoid damage from light stress via movement of chloroplasts, change in leaf area and leaf angle, adjustment of antenna size, regulation of thermal dissipation, and scavenging reactive oxygen species to prevent oxidative stress (Takahashi and Murata, 2008; Murchie and Niyogi, 2011). The thermal dissipation can be measured as quenching of chlorophyll fluorescence with the common term of non- 
photochemical quenching (NPQ) (Baker, 2008). From NPQ measurements, several parameters can be extracted to get the insight of energy dependence quenching $\left(q_{E}\right)$, photoinhibition of photosynthesis $\left(q_{l}\right)$, quenching coefficient by non-photochemical process $(q M)$, photochemical coefficient quenching $(q P)$, and quenching of NPQ during illumination (qL) (Lazár, 2015).

Prolonged photoperiod $(16.5 \mathrm{~h}$ ) led to light excess and influenced the photosynthesis performance of diploids and tetraploids. Diploids were more sensitive to stress compared to polyploids, as indicated by the alternation of non-photochemical quenching parameters ( $q_{1}$ and $q M$. Tetraploids experienced a lesser stress effect as indicated by an alternation of $q P$ and $q L$ parameters which means the light excess is quenched by photochemistry, and the fraction of light is dissipated into heat (Lazár, 2015).

The photosynthesis performance also observed by fast chlorophyll a fluorescence transient which provides that the fluorescence rise is related to the closure of some PSIl reaction centers (Kalaji et al., 2016); the parameters of performance index on absorption basis (PI_Abs) represent the sum of structural and functional criteria of absorption flux per reaction center $(A B S / R C)$, trapped energy flux per $R C\left(T R_{0} / R C\right)$, electron transport flux per reaction center $(\mathrm{ET} / \mathrm{RC})$, and Dissipated energy flux per reaction center ( $\left.\mathrm{DI}_{0} / \mathrm{RC}\right)$ (Brestic and Zivcak, 2013; Stirbet et al., 2018). The stress altered the performance index on an absorption basis in diploids. In diploids, the light stress influenced the antenna membrane resulted in a massive closure of the PSIl reaction centers (ABS/RC), reduced the capacity of photon trapping $\left(E T_{0} / R C\right)$, and increased the photon dissipation ( $\left.\mathrm{DI}_{0} / \mathrm{RC}\right)$. In tetraploids, the lesser stress level might not have affected the antenna molecule in the photosynthetic membrane, but the fraction of light exceeding the capacity of photon trapping $\left(E T_{0} / R C\right)$, hence dissipated into nonphotochemical quenching $(q L)$.

The three cytotypes of $R$. auricomus had been raised from seedling in garden conditions and kept in cultivated for several years (Horandl et al., 1997; Paun et al., 2006; Hodač et al., 2014; Hojsgaard et al., 2014a; Barke et al., 2018), but their photosynthesis activity still followed the pre-adaptation to habitats of their mother plants from natural sites as observed in relative electron transport rate curves and photosynthesis efficiency parameters. The light intensity of our climatic chamber, i.e., c.a. $250 \mu \mathrm{mol}$ photons $\mathrm{m}^{-2} \mathrm{~s}^{-1}$ provided optimal condition to the plants as indicated by the absence of photoinhibition in all three cytotypes, except for hexaploid clone 35 as indicated by parameter PSII maximum efficiency ( $\phi P S I I)$ and maximum quantum efficiency of PSII photochemistry (QY_max). Measurement of relative electron curves revealed that diploids and hexaploid clone 29 were more adaptive to a wide range of light intensity. On the other hand, tetraploids performed better photosynthesis under lower 
light intensity (below $300 \mu \mathrm{mol}$ photons $\mathrm{m}^{-2} \mathrm{~s}^{-1}$ at PPFD), whereas hexaploid clone 35 performed better photosynthesis under higher light intensity (more than $300 \mu \mathrm{mol}$ photons $\mathrm{m}^{-}$ ${ }^{2} \mathrm{~s}^{-1}$ at PPFD). The contrasts of photosynthesis capacity of hexaploid clones might be related to the high level of heterozygosity in polyploidy, as reported recently (Karbstein et al., 2021). The advantage of heterozygosity is the potential for diversifying the gene function by altering redundant copies of important or essential genes (Comai, 2005). An example was presented in tetraploid Glycine max with overexpression of oxidative stress-regulating genes compared to the diploid progenitors, which correlated to differential photosynthetic performance and adaptation to higher light intensities (Coate et al., 2012). Epigenetic studies on polyploids of $R$. auricomus will be beneficial to combine the current result with the phenotypic variation among hexaploid clones and among cytotypes. In diploid and tetraploid $R$. kuepferi a study on cytosin-methylation indicated different epigenetic profiles within cytotype and between habitats (Schinkel et al., 2020). 


\section{References}

Abuelsoud, W., Cortleven, A., and Schmulling, T. (2020). Photoperiod stress alters the cellular redox status and is associated with an increased peroxidase and decreased catalase activity. bioRxiv. doi: 10.1101/2020.03.05.978270.

Acuña-Rodríguez, I.S., Newsham, K.K., Gundel, P.E., Torres-Díaz, C., and MolinaMontenegro, M.A. (2020). Functional roles of microbial symbionts in plant cold tolerance. Ecology Letters 23(6), 1034-1048. doi: https://doi.org/10.1111/ele.13502.

Alix, K., Gérard, P.R., Schwarzacher, T., and Heslop-Harrison, J.S. (2017). Polyploidy and interspecific hybridization: partners for adaptation, speciation and evolution in plants. Annals of Botany 120(2), 183-194. doi: 10.1093/aob/mcx079.

Aliyu, O.M., Schranz, M.E., and Sharbel, T.F. (2010). Quantitative variation for apomictic reproduction in the genus Boechera (Brassicaceae). American Journal of Botany 97(10), 1719-1731.

Anneberg, T.J., and Segraves, K.A. (2019). Intraspecific polyploidy correlates with colonization by arbuscular mycorrhizal fungi in Heuchera cylindrica. American Journal of Botany 106(6), 894-900. doi: https://doi.org/10.1002/ajb2.1294.

Asker, S.E., and Jerling, L. (1992). Apomixis in plants. CRC Press.

Baker, N.R. (2008). Chlorophyll fluorescence: a probe of photosynthesis in vivo. Annu. Rev. Plant Biol. 59, 89-113. doi: 10.1146/annurev.arplant.59.032607.092759.

Baniaga, A.E., Marx, H.E., Arrigo, N., and Barker, M.S. (2020). Polyploid plants have faster rates of multivariate niche differentiation than their diploid relatives. Ecology Letters 23(1), 68-78. doi: https://doi.org/10.1111/ele.13402.

Barke, B.H., Daubert, M., and Hörandl, E. (2018). Establishment of Apomixis in Diploid F2 Hybrids and Inheritance of Apospory From F1 to F2 Hybrids of the Ranunculus auricomus Complex. Frontiers in Plant Science 9(1111). doi: 10.3389/fpls.2018.01111.

Barke, B.H., Karbstein, K., Daubert, M., and Hörandl, E. (2020). The relation of meiotic behaviour to hybridity, polyploidy and apomixis in the Ranunculus auricomus complex (Ranunculaceae). BMC Plant Biology 20(1), 523. doi: 10.1186/s12870-020-02654-3.

Bauerle, W.L., Oren, R., Way, D.A., Qian, S.S., Stoy, P.C., Thornton, P.E., et al. (2012). Photoperiodic regulation of the seasonal pattern of photosynthetic capacity and the implications for carbon cycling. Proceedings of the National Academy of Sciences 109(22), 8612-8617. doi: 10.1073/pnas.1119131109.

Bicknell, R.A., and Koltunow, A.M. (2004). Understanding apomixis: recent advances and remaining conundrums. The Plant Cell 16(suppl 1), S228-S245. doi: 10.1105/tpc.017921.

Bicknell, R.A., Lambie, S.C., and Butler, R.C. (2003). Quantification of progeny classes in two facultatively apomictic accessions of Hieracium. Hereditas 138(1), 11-20. doi: 10.1034/j.1601-5223.2003.01624.x.

Björkman, O., and Demmig, B. (1987). Photon yield of $\mathrm{O}_{2}$ evolution and chlorophyll fluorescence characteristics at $77 \mathrm{~K}$ among vascular plants of diverse origins. Planta 170(4), 489-504. doi: 10.1007/BF00402983.

Bolker, B., and Bolker, M.B. (2020). " Package'bbmle'.Tools for General Maximum Likelihood Estimation,641

Bordenave, C.D., Rocco, R., Maiale, S.J., Campestre, M.P., Ruiz, O.A., Rodríguez, A.A., et al. (2019). Chlorophyll a fluorescence analysis reveals divergent photosystem II responses to saline, alkaline and saline-alkaline stresses in the two Lotus japonicus 
model ecotypes MG20 and Gifu-129. Acta Physiologiae Plantarum 41(9), 1-13. doi: 10.1007/s11738-019-2956-0.

Brestic, M., and Zivcak, M. (2013). PSIl fluorescence techniques for measurement of drought and high temperature stress signal in crop plants: protocols and applications. Molecular stress physiology of plants, 87-131. doi: 10.1007/978-81-322-0807-5.

Brochmann, C., Brysting, A.K., Alsos, I.G., Borgen, L., Grundt, H.H., Scheen, A.-C., et al. (2004). Polyploidy in arctic plants. Biological Journal of the Linnean Society 82(4), 521 536. doi: 10.1111/j.1095-8312.2004.00337.x.

Brooks, M.E., Kristensen, K., van Benthem, K.J., Magnusson, A., Berg, C.W., Nielsen, A., et al. (2017). glmmTMB balances speed and flexibility among packages for zero-inflated generalized linear mixed modeling. The $R$ journal 9(2), 378-400. doi: 10.32614/RJ2017-066.

Carillo, P., Arena, C., Modarelli, G.C., De Pascale, S., and Paradiso, R. (2019). Photosynthesis in Ranunculus asiaticus L.: the influence of the hybrid and the preparation procedure of tuberous roots. Frontiers in plant science 10, 241. doi: 10.3389/fpls.2019.00241.

Carman, J.G. (1997). Asynchronous expression of duplicate genes in angiosperms may cause apomixis, bispory, tetraspory, and polyembryony. Biological Journal of the Linnean Society 61(1), 51-94.

Carman, J.G., Mateo de Arias, M., Gao, L., Zhao, X., Kowallis, B.M., Sherwood, D.A., et al. (2019). Apospory and Diplospory in Diploid Boechera (Brassicaceae) May Facilitate Speciation by Recombination-Driven Apomixis-to-Sex Reversals. Frontiers in Plant Science 10(724). doi: 10.3389/fpls.2019.00724.

Chao, D.-Y., Dilkes, B., Luo, H., Douglas, A., Yakubova, E., Lahner, B., et al. (2013). Polyploids exhibit higher potassium uptake and salinity tolerance in Arabidopsis. Science 341(6146), 658-659.

Çiçek, N., Kalaji, H.M., and Ekmekçi, Y. (2020). Special issue in honour of Prof. Reto J. Strasser-Probing the photosynthetic efficiency of some European and Anatolian Scots pine populations under UV-B radiation using polyphasic chlorophyll a fluorescence transient. Photosynthetica 58(SPECIAL ISSUE), 468-478. doi: 10.32615/ps.2019.151.

Coate, J.E., Luciano, A.K., Seralathan, V., Minchew, K.J., Owens, T.G., and Doyle, J.J. (2012). Anatomical, biochemical, and photosynthetic responses to recent allopolyploidy in Glycine dolichocarpa (Fabaceae). American Journal of Botany 99(1), 55-67. doi: doi.org/10.3732/ajb.1100465.

Coate, J.E., Powell, A.F., Owens, T.G., and Doyle, J.J. (2013). Transgressive physiological and transcriptomic responses to light stress in allopolyploid Glycine dolichocarpa (Leguminosae). Heredity 110(2), 160-170. doi: 10.1038/hdy.2012.77.

Comai, L. (2005). The advantages and disadvantages of being polyploid. Nature reviews genetics 6(11), 836.

Cosendai, A.-C., and Hörandl, E. (2010). Cytotype stability, facultative apomixis and geographical parthenogenesis in Ranunculus kuepferi (Ranunculaceae). Annals of Botany 105(3), 457-470.

D'Ambrosio, N., Guadagno, C., and De Santo, A.V. (2008). Is qE always the major component of non-photochemical quenching? Photosynthesis. Energy from the sun, 1001-1004. doi: 10.1007/978-1-4020-6709-9_218.

de Castro, K.M., Batista, D.S., Fortini, E.A., Silva, T.D., Felipe, S.H.S., Fernandes, A.M., et al. (2019). Photoperiod modulates growth, morphoanatomy, and linalool content in Lippia 
alba L.(Verbenaceae) cultured in vitro. Plant Cell, Tissue and Organ Culture (PCTOC) 139(1), 139-153. doi: 10.1007/s11240-019-01672-w.

De Storme, N., and Geelen, D. (2014). The impact of environmental stress on male reproductive development in plants: biological processes and molecular mechanisms. Plant, Cell \& Environment 37(1), 1-18. doi: https://doi.org/10.1111/pce.12142.

del Pozo, J.C., and Ramirez-Parra, E. (2014). Deciphering the molecular bases for drought tolerance in Arabidopsis autotetraploids. Plant, Cell \& Environment 37(12), 2722-2737.

Delgado, L., Sartor, M.E., Espinoza, F., Soliman, M., Galdeano, F., and Ortiz, J.P.A. (2016). Hybridity and autopolyploidy increase the expressivity of apospory in diploid Paspalum rufum. Plant Systematics and Evolution 302(10), 1471-1481. doi: 10.1007/s00606016-1345-z.

Demmig-Adams, B., and Adams III, W.W. (2006). Photoprotection in an ecological context: the remarkable complexity of thermal energy dissipation. New Phytologist 172(1), 1121. doi: 10.1111/j.1469-8137.2006.01835.x.

Deng, B., Du, W., Liu, C., Sun, W., Tian, S., and Dong, H. (2012). Antioxidant response to drought, cold and nutrient stress in two ploidy levels of tobacco plants: low resource requirement confers polytolerance in polyploids? Plant Growth Regulation 66(1), 3747. doi: 10.1007/s10725-011-9626-6.

Dobeš, C., Scheffknecht, S., Fenko, Y., Prohaska, D., Sykora, C., and Hülber, K. (2018). Asymmetric reproductive interference: The consequences of cross-pollination on reproductive success in sexual-apomictic populations of Potentilla puberula (Rosaceae). Ecology and evolution 8(1), 365-381.

Doyle, J.J., and Coate, J.E. (2019). Polyploidy, the Nucleotype, and Novelty: The Impact of Genome Doubling on the Biology of the Cell. International Journal of Plant Sciences 180(1), 1-52. doi: 10.1086/700636.

Duarte, B., Gössling, J.W., Marques, J., and Caçador, I. (2015). Ecophysiological constraints of Aster tripolium under extreme thermal events impacts: merging biophysical, biochemical and genetic insights. Plant Physiology and Biochemistry 97, 217-228. doi: 10.1016/j.plaphy.2015.10.015.

Elkins, C., and van lersel, M.W. (2020). Longer photoperiods with the same daily light integral improve growth of Rudbeckia seedlings in a greenhouse. HortScience 1(aop), 1-7. doi: 10.21273/HORTSCI15200-20.

Evans, L., and Knox, R. (1969). Environmental control of reproduction in Themeda australis. Australian Journal of Botany 17(3), 375-389.

Ferrari, S., and Cribari-Neto, F. (2004). Beta regression for modelling rates and proportions. Journal of applied statistics 31(7), 799-815. doi: 10.1080/0266476042000214501.

Fortini, E.A., Batista, D.S., de Castro, K.M., Silva, T.D., Felipe, S.H.S., Correia, L.N.F., et al. (2020). Photoperiod modulates growth and pigments and 20-hydroxyecdysone accumulation in Brazilian ginseng Pfaffia glomerata (Spreng.) Pedersen] grown in vitro. Plant Cell, Tissue and Organ Culture (PCTOC) 142(3), 595-611. doi: 10.1007/s11240-020-01886-3.

Foyer, C.H., and Noctor, G. (2009). Redox regulation in photosynthetic organisms: signaling, acclimation, and practical implications. Antioxidants \& redox signaling 11(4), 861-905.

Fréchette, E., Chang, C.Y.-Y., and Ensminger, I. (2016). Photoperiod and temperature constraints on the relationship between the photochemical reflectance index and the light use efficiency of photosynthesis in Pinus strobus. Tree physiology 36(3), 311-324. doi: $10.1093 /$ treephys/tpv143. 
Gao, L. (2018). Pharmacologically Induced Meiosis Apomeiosis Interconversions in Boechera, Arabidopsis and Vigna. Doctor of Philosophy (PhD) Dissertation, Utah State University.

Genty, B., Briantais, J.-M., and Baker, N.R. (1989). The relationship between the quantum yield of photosynthetic electron transport and quenching of chlorophyll fluorescence. Biochimica et Biophysica Acta (BBA)-General Subjects 990(1), 87-92. doi: 10.1016/S0304-4165(89)80016-9.

Ghashghaie, G.C.J., Genty, B., and Briantais, J. (1992). Leaf photosynthesis is resistant to a mild drought stress. Photosynthetica 27(3), 295-309. doi: 10.1007/0-306-48135-9_14.

Godfree, R.C., Marshall, D.J., Young, A.G., Miller, C.H., and Mathews, S. (2017). Empirical evidence of fixed and homeostatic patterns of polyploid advantage in a keystone grass exposed to drought and heat stress. Royal Society open science 4(11), 170934.

Gounaris, E., Sherwood, R., Gounaris, I., Hamilton, R., and Gustine, D. (1991). Inorganic salts modify embryo sac development in sexual and aposporous Cenchrus ciliaris. Sexual Plant Reproduction 4(3), 188-192.

Grant, V. (1981). "Plant speciation". Columbia University Press).

Grimanelli, D. (2012). Epigenetic regulation of reproductive development and the emergence of apomixis in angiosperms. Current Opinion in Plant Biology 15(1), 57-62. doi: https://doi.org/10.1016/j.pbi.2011.10.002.

Grimanelli, D., Hernández, M., Perotti, E., and Savidan, Y. (1997). Dosage effects in the endosperm of diplosporous apomictic Tripsacum (Poaceae). Sexual Plant Reproduction 10(5), 279-282.

Gururani, M.A., Venkatesh, J., and Tran, L.S.P. (2015). Regulation of photosynthesis during abiotic stress-induced photoinhibition. Molecular plant 8(9), 1304-1320. doi: 10.1016/j.molp.2015.05.005.

Halliwell, B. (2006). Reactive species and antioxidants. Redox biology is a fundamental theme of aerobic life. Plant physiology 141(2), 312-322.

Hand, M.L., and Koltunow, A.M. (2014). The genetic control of apomixis: asexual seed formation. Genetics 197(2), 441-450.

Hannweg, K., Steyn, W., and Bertling, I. (2016). In vitro-induced tetraploids of Plectranthus esculentus are nematode-tolerant and have enhanced nutritional value. Euphytica 207(2), 343-351. doi: 10.1007/s10681-015-1547-4.

Harms, N., Shearer, J., Cronin, J.T., and Gaskin, J.F. (2020). Geographic and genetic variation in susceptibility of Butomus umbellatus to foliar fungal pathogens. Biological Invasions 22(2), 535-548. doi: 10.1007/s10530-019-02109-3.

Hartig, F., and Hartig, M.F. (2017). "Package 'DHARMa'". 0.4.1. https://cran.rproject.org/web/packages/DHARMa/vignettes/DHARMa.html

Hias, N., Leus, L., Davey, M.W., Vanderzande, S., Van Huylenbroeck, J., and Keulemans, J. (2017). Effect of polyploidization on morphology in two apple (Malusx domestica) genotypes. Horticultural Science 44(2), 55-63.

Hodač, L., Klatt, S., Hojsgaard, D., Sharbel, T.F., and Hörandl, E. (2019). A little bit of sex prevents mutation accumulation even in apomictic polyploid plants. BMC evolutionary biology 19(1), 1-11.

Hodač, L., Scheben, A.P., Hojsgaard, D., Paun, O., and Hörandl, E. (2014). ITS Polymorphisms Shed Light on Hybrid Evolution in Apomictic Plants: A Case Study on the Ranunculus auricomus Complex. PLOS ONE 9(7), e103003. doi: 10.1371/journal.pone.0103003. 
Hojsgaard, D. (2018). Transient Activation of Apomixis in Sexual Neotriploids May Retain Genomically Altered States and Enhance Polyploid Establishment. Frontiers in Plant Science 9(230). doi: 10.3389/fpls.2018.00230.

Hojsgaard, D., Greilhuber, J., Pellino, M., Paun, O., Sharbel, T.F., and Hörandl, E. (2014a). Emergence of apospory and bypass of meiosis via apomixis after sexual hybridisation and polyploidisation. New Phytologist 204(4), 1000-1012. doi: 10.1111/nph.12954.

Hojsgaard, D., and Hörandl, E. (2019). The Rise of Apomixis in Natural Plant Populations. Frontiers in plant science 10, 358-358. doi: 10.3389/fpls.2019.00358.

Hojsgaard, D., Klatt, S., Baier, R., Carman, J.G., and Hörandl, E. (2014b). Taxonomy and biogeography of apomixis in angiosperms and associated biodiversity characteristics. Critical Reviews in Plant Sciences 33(5), 414-427.

Hojsgaard, D.H., Martínez, E.J., and Quarin, C.L. (2013). Competition between meiotic and apomictic pathways during ovule and seed development results in clonality. New Phytologist 197(1), 336-347.

Hörandl, E. (1998). Species concepts in agamic complexes: Applications in the Ranunculus auricomus complex and general perspectives. Folia Geobotanica 33(3), 335-348. doi: 10.1007/BF03216210.

Hörandl, E. (2008). Evolutionary implications of self-compatibility and reproductive fitness in the apomictic Ranunculus auricomus polyploid complex (ranunculaceae). International journal of plant sciences 169(9), 1219-1228. doi: 10.1086/591980.

Horandl, E., Dobes, C., and Lambrou, M. (1997). Chromosome and pollen studies on Austrian species of the apomictic Ranunculus auricomus complex. Botanica Helvetica 107(2), 195-209.

Hörandl, E., Greilhuber, J., Klímová, K., Paun, O., Temsch, E., Emadzade, K., et al. (2009). Reticulate evolution and taxonomic concepts in the Ranunculus auricomus complex (Ranunculaceae): insights from analysis of morphological, karyological and molecular data. Taxon 58(4), 1194-1216. doi: 10.1002/tax.584012.

Hörandl, E., and Hadacek, F. (2013). The oxidative damage initiation hypothesis for meiosis. Plant reproduction 26(4), 351-367.

Hörandl, E., and Temsch, E.M. (2009). Introgression of apomixis into sexual species is inhibited by mentor effects and ploidy barriers in the Ranunculus auricomus complex. Annals of Botany 104(1), 81-89.

Huang, H., Ullah, F., Zhou, D.-X., Yi, M., and Zhao, Y. (2019). Mechanisms of ROS regulation of plant development and stress responses. Frontiers in Plant Science 10.

lannicelli, J., Guariniello, J., Tossi, V.E., Regalado, J.J., Di Ciaccio, L., Van Baren, C.M., et al. (2020). The "polyploid effect" in the breeding of aromatic and medicinal species. Scientia Horticulturae 260, 108854. doi: 10.1016/j.scienta.2019.108854.

Izmaiłow, R. (1996). Reproductive strategy in the Ranunculus auricomus complex (Ranunculaceae). Acta Societatis Botanicorum Poloniae 65(1-2), 167-170.

Jackson, S.D. (2009). Plant responses to photoperiod. New Phytologist 181(3), 517-531. doi: 10.1111/j.1469-8137.2008.02681.x.

Jeong, S., and Clark, S.E. (2005). Photoperiod regulates flower meristem development in Arabidopsis thaliana. Genetics 169(2), 907-915. doi: 10.1534/genetics.104.033357.

Jiang, J., Yuan, Y., Zhu, S., Fang, T., Ran, L., Wu, J., et al. (2019). Comparison of physiological and methylational changes in resynthesized Brassica napus and diploid progenitors under drought stress. Acta Physiologiae Plantarum 41(4), 45. 
Juranić, M., Tucker, M.R., Schultz, C.J., Shirley, N.J., Taylor, J.M., Spriggs, A., et al. (2018). Asexual female gametogenesis involves contact with a sexually-fated megaspore in apomictic Hieracium. Plant physiology 177(3), 1027-1049. doi: 10.1104/pp.18.00342.

Kalaji, H.M., Jajoo, A., Oukarroum, A., Brestic, M., Zivcak, M., Samborska, I.A., et al. (2016). Chlorophyll a fluorescence as a tool to monitor physiological status of plants under abiotic stress conditions. Acta Physiologiae Plantarum 38(4), 102. doi: 10.1007/s11738-016-2113-y.

Karbstein, K., Tomasello, S., Hodač, L., Lorberg, E., Daubert, M., and Hörandl, E. (2021). Moving beyond assumptions: polyploidy and environmental effects explain a geographical parthenogenesis scenario in European plants. Molecular Ecology. doi: 10.1111/mec.15919.

Karunarathne, P., Reutemann, A.V., Schedler, M., Glücksberg, A., Martínez, E.J., Honfi, A.I., et al. (2020). Sexual modulation in a polyploid grass: a reproductive contest between environmentally inducible sexual and genetically dominant apomictic pathways. Scientific reports 10(1), 1-14. doi: 10.1038/s41598-020-64982-6.

Kaushal, P., Dwivedi, K.K., Radhakrishna, A., Saxena, S., Paul, S., Srivastava, M.K., et al. (2018). Ploidy dependent expression of apomixis and its components in guinea grass (Panicum maximum Jacq.). Euphytica 214(9), 152. doi: 10.1007/s10681-018-2232-1.

Kautsky, H., and Hirsch, A. (1931). Neue Versuche zur Kohlensäureassimilation. Naturwissenschaften 19(48), 964-964. doi: 10.1007/BF01516164.

Keane, O.M., Toft, C., Carretero-Paulet, L., Jones, G.W., and Fares, M.A. (2014). Preservation of genetic and regulatory robustness in ancient gene duplicates of Saccharomyces cerevisiae. Genome Research 24(11), 1830-1841.

Keeney, S., Lange, J., and Mohibullah, N. (2014). Self-organization of meiotic recombination initiation: general principles and molecular pathways. Annual review of genetics 48, 187-214.

Kinoshita, T., Kume, A., and Hanba, Y.T. (2020). Seasonal variations in photosynthetic functions of the urban landscape tree species Gingko biloba: photoperiod is a key trait. Trees, 1-13. doi: 10.1007/s00468-020-02033-3.

Klatt, S., Hadacek, F., Hodač, L., Brinkmann, G., Eilerts, M., Hojsgaard, D., et al. (2016). Photoperiod extension enhances sexual megaspore formation and triggers metabolic reprogramming in facultative apomictic Ranunculus auricomus. Frontiers in plant science 7, 278.

Klatt, S., Schinkel, C.C., Kirchheimer, B., Dullinger, S., and Hörandl, E. (2018). Effects of cold treatments on fitness and mode of reproduction in the diploid and polyploid alpine plant Ranunculus kuepferi (Ranunculaceae). Annals of botany 121(7), 1287-1298.

Knox, R. (1967). Apomixis: seasonal and population differences in a grass. Science 157(3786), 325-326.

Koltunow, A.M. (1993). Apomixis: embryo sacs and embryos formed without meiosis or fertilization in ovules. The plant cell 5(10), 1425. doi: 10.1105/tpc.5.10.1425.

Koltunow, A.M., and Grossniklaus, U. (2003). Apomixis: a developmental perspective. Annual review of plant biology 54(1), 547-574.

Kramer, D.M., Johnson, G., Kiirats, O., and Edwards, G.E. (2004). New fluorescence parameters for the determination of $Q_{A}$ redox state and excitation energy fluxes. Photosynthesis research 79(2), 209-218. doi: 10.1023/B:PRES.0000015391.99477.0d. 
Krause, G., and Weis, E. (1991). Chlorophyll fluorescence and photosynthesis: the basics. Annual review of plant biology 42(1), 313-349. doi: 10.1146/annurev.pp.42.060191.001525.

Landis, J.B., Soltis, D.E., Li, Z., Marx, H.E., Barker, M.S., Tank, D.C., et al. (2018). Impact of whole-genome duplication events on diversification rates in angiosperms. American journal of botany 105(3), 348-363. doi: 10.1002/ajb2.1060.

Lavaud, J. (2007). Fast regulation of photosynthesis in diatoms: mechanisms, evolution and ecophysiology. Functional Plant Science and Biotechonology, 21, 267-287.

Lazár, D. (2015). Parameters of photosynthetic energy partitioning. Journal of Plant Physiology 175, 131-147. doi: 10.1016/j.jplph.2014.10.021.

Liu, B., and Sun, G. (2019). Transcriptome and miRNAs analyses enhance our understanding of the evolutionary advantages of polyploidy. Critical reviews in biotechnology 39(2), 173-180.

Lourkisti, R., Froelicher, Y., Herbette, S., Morillon, R., Tomi, F., Gibernau, M., et al. (2020). Triploid citrus genotypes have a better tolerance to natural chilling conditions of photosynthetic capacities and specific leaf volatile organic compounds. Frontiers in plant science 11, 330. doi: 10.3389/fpls.2020.00330.

Ma, X., Su, Z., and Ma, H. (2020). Molecular genetic analyses of abiotic stress responses during plant reproductive development. Journal of Experimental Botany 71(10), 28702885. doi: 10.1093/jxb/eraa089.

Martínez, L.M., Fernández-Ocaña, A., Rey, P.J., Salido, T., Amil-Ruiz, F., and Manzaneda, A.J. (2018). Variation in functional responses to water stress and differentiation between natural allopolyploid populations in the Brachypodium distachyon species complex. Annals of Botany 121(7), 1369-1382. doi: 10.1093/aob/mcy037.

Masterson, J. (1994). Stomatal Size in Fossil Plants: Evidence for Polyploidy in Majority of Angiosperms. Science 264(5157), 421-424. doi: 10.1126/science.264.5157.421.

Mateo de Arias, M. (2015). Effects of plant stress on facultative apomixis in Boechera (Brassicaceae). Doctor of Philosophy (PhD) Dissertation, Utah State University.

Mateo de Arias, M., Gao, L., Sherwood, D.A., Dwivedi, K.K., Price, B.J., Jamison, M., et al. (2020). Whether gametophytes are reduced or unreduced in angiosperms might be determined metabolically. Genes 11(12), 1449. doi: doi.org/10.3390/genes11121449.

Maxwell, K., and Johnson, G.N. (2000). Chlorophyll fluorescence-a practical guide. Journal of experimental botany 51(345), 659-668. doi: 10.1093/jexbot/51.345.659.

Milev, N.B., and Reddy, A.B. (2015). Circadian redox oscillations and metabolism. Trends in Endocrinology \& Metabolism 26(8), 430-437. doi: 10.1016/j.tem.2015.05.012.

Mogie, M. (1992). The evolution of asexual reproduction in plants. London: Chapman and Hall.

Mráz, P., Chrtek, J., and Šingliarová, B. (2009). Geographical parthenogenesis, genome size variation and pollen production in the arctic-alpine species Hieracium alpinum. Botanica Helvetica 119(1), 41-51.

Müller, P., Li, X.-P., and Niyogi, K.K. (2001). Non-photochemical quenching. A response to excess light energy. Plant physiology 125(4), 1558-1566. doi: 10.1104/pp.125.4.1558.

Mungoma, C. (1988). Photoperiod sensitivity in tropical maize accessions, early inbreds, and their crosses. lowa State University.

Münzbergová, Z., and Haisel, D. (2019). Effects of polyploidization on the contents of photosynthetic pigments are largely population-specific. Photosynthesis research 140(3), 289-299. doi: 10.1007/s11120-018-0604-y. 
Murchie, E.H., and Lawson, T. (2013). Chlorophyll fluorescence analysis: a guide to good practice and understanding some new applications. Journal of experimental botany 64(13), 3983-3998. doi: 10.1093/jxb/ert208.

Murchie, E.H., and Niyogi, K.K. (2011). Manipulation of photoprotection to improve plant photosynthesis. Plant physiology 155(1), 86-92. doi: 10.1104/pp.110.168831.

Nitschke, S., Cortleven, A., Iven, T., Feussner, I., Havaux, M., Riefler, M., et al. (2016). Circadian Stress Regimes Affect the Circadian Clock and Cause Jasmonic AcidDependent Cell Death in Cytokinin-Deficient Arabidopsis Plants. The Plant Cell 28(7), 1616-1639. doi: 10.1105/tpc. 16.00016.

Nogler, G. (1984a). "Gametophytic apomixis," in Embryology of angiosperms. Springer, 475518.

Nogler, G. (1984b). Genetics of apospory in Ranunculus auricomus V, Conclusions. Bot. Helv. 94, 411-422.

Obata, T., and Fernie, A.R. (2012). The use of metabolomics to dissect plant responses to abiotic stresses. Cellular and Molecular Life Sciences 69(19), 3225-3243. doi: 10.1007/s00018-012-1091-5.

Orians, C.M. (2000). The effects of hybridization in plants on secondary chemistry: implications for the ecology and evolution of plant-herbivore interactions. American Journal of Botany 87(12), 1749-1756. doi: 10.2307/2656824.

Otto, F. (1990). "Chapter 11 DAPI Staining of Fixed Cells for High-Resolution Flow Cytometry of Nuclear DNA," in Methods in Cell Biology, eds. Z. Darzynkiewicz \& H.A. Crissman. Academic Press), 105-110.

Otto, S.P. (2007). The Evolutionary Consequences of Polyploidy. Cell 131(3), 452-462. doi: https://doi.org/10.1016/j.cell.2007.10.022.

Oustric, J., Morillon, R., Ollitrault, P., Herbette, S., Luro, F., Froelicher, Y., et al. (2018). Somatic hybridization between diploid Poncirus and Citrus improves natural chilling and light stress tolerances compared with equivalent doubled-diploid genotypes. Trees 32(3), 883-895. doi: 10.1007/s00468-018-1682-3.

Ozias-Akins, P., and van Dijk, P.J. (2007). Mendelian genetics of apomixis in plants. Annu. Rev. Genet. 41, 509-537.

Paun, O., Greilhuber, J., Temsch, E., and Hörandl, E. (2006). Patterns, sources and ecological implications of clonal diversity in apomictic Ranunculus carpaticola (Ranunculus auricomus complex, Ranunculaceae). Molecular ecology 15(4), 897-910. doi: 10.1111/j.1365-294X.2006.02800.x.

Paupière, M.J., Müller, F., Li, H., Rieu, I., Tikunov, Y.M., Visser, R.G., et al. (2017). Untargeted metabolomic analysis of tomato pollen development and heat stress response. Plant reproduction 30(2), 81-94. doi: 10.1007/s00497-017-0301-6.

Porcar-Castell, A., Tyystjärvi, E., Atherton, J., Van der Tol, C., Flexas, J., Pfündel, E.E., et al. (2014). Linking chlorophyll a fluorescence to photosynthesis for remote sensing applications: mechanisms and challenges. Journal of experimental botany 65(15), 4065-4095. doi: 10.1093/jxb/eru191.

Ptushenko, V.V., Ptushenko, E.A., Samoilova, O.P., and Tikhonov, A.N. (2013). Chlorophyll fluorescence in the leaves of Tradescantia species of different ecological groups: induction events at different intensities of actinic light. Biosystems 114(2), 85-97. doi: 10.1016/j.biosystems.2013.08.001.

Quarin, C.L. (1986). Seasonal changes in the incidence of apomixis of diploid, triploid, and tetraploid plants of Paspalum cromyorrhizon. Euphytica 35(2), 515-522. doi: 10.1007/bf00021860. 
Quarin, C.L. (1999). Effect of pollen source and pollen ploidy on endosperm formation and seed set in pseudogamous apomictic Paspalum notatum. Sexual Plant Reproduction 11(6), 331-335.

Ralph, P.J., and Gademann, R. (2005). Rapid light curves: a powerful tool to assess photosynthetic activity. Aquatic botany 82(3), 222-237. doi: 10.1016/j.aquabot.2005.02.006.

Ram, Y., and Hadany, L. (2016). Condition-dependent sex: who does it, when and why? Philosophical Transactions of the Royal Society B: Biological Sciences 371(1706), 20150539.

Rambaut, A. (2007). "FigTree". v1.3.1. Available: http://tree.bio.ed.ac.uk/software/figtree/ [Accessed 2017]

Ramsey, J., and Schemske, D.W. (1998). Pathways, mechanisms, and rates of polyploid formation in flowering plants. Annual Review of Ecology and Systematics 29(1), 467501. doi: 10.1146/annurev.ecolsys.29.1.467.

Rao, S., Tian, Y., Xia, X., Li, Y., and Chen, J. (2020). Chromosome doubling mediates superior drought tolerance in Lycium ruthenicum via abscisic acid signaling. Horticulture research 7(1), 1-18. doi: 10.1038/s41438-020-0260-1.

Rice, A., Šmarda, P., Novosolov, M., Drori, M., Glick, L., Sabath, N., et al. (2019). The global biogeography of polyploid plants. Nature Ecology \& Evolution 3(2), 265-273. doi: 10.1038/s41559-018-0787-9.

Ripoll, J., Bertin, N., Bidel, L.P., and Urban, L. (2016). A user's view of the parameters derived from the induction curves of maximal chlorophyll a fluorescence: perspectives for analyzing stress. Frontiers in plant science 7, 1679. doi: 10.3389/fpls.2016.01679.

Rodrigo, J.M., Zappacosta, D.C., Selva, J.P., Garbus, I., Albertini, E., and Echenique, V. (2017). Apomixis frequency under stress conditions in weeping lovegrass (Eragrostis curvula). PLOS ONE 12(4), e0175852. doi: 10.1371/journal.pone.0175852.

Roeber, V.M., Bajaj, I., Rohde, M., Schmülling, T., and Cortleven, A. (2021). Light acts as a stressor and influences abiotic and biotic stress responses in plants. Plant, Cell \& Environment 44(3), 645-664. doi: https://doi.org/10.1111/pce.13948.

Roháček, K., Soukupová, J., and Barták, M. (2008). Chlorophyll fluorescence: a wonderful tool to study plant physiology and plant stress. Plant Cell Compartments-Selected Topics. Research Signpost, Kerala, India, 41-104. doi: 10.1007/s13580-014-0006-9.

Rusaczonek, A., Czarnocka, W., Kacprzak, S., Witoń, D., Ślesak, I., Szechyńska-Hebda, M., et al. (2015). Role of phytochromes A and B in the regulation of cell death and acclimatory responses to UV stress in Arabidopsis thaliana. Journal of experimental botany 66(21), 6679-6695. doi: 10.1093/jxb/erv375

Saran, S., and de Wet, J. (1976). Environmental-control of reproduction in Dichanthiumintermedium. Journal of Cytology and Genetics 11, 22-28.

Šarhanová, P., Vašut, R.J., Dančák, M., Bureš, P., and Trávníček, B. (2012). New insights into the variability of reproduction modes in European populations of Rubus subgen. Rubus: how sexual are polyploid brambles? Sexual Plant Reproduction 25(4), 319335. doi: 10.1007/s00497-012-0200-9.

Sato, S., Kamiyama, M., Iwata, T., Makita, N., Furukawa, H., and Ikeda, H. (2006). Moderate increase of mean daily temperature adversely affects fruit set of Lycopersicon esculentum by disrupting specific physiological processes in male reproductive development. Annals of Botany 97(5), 731-738. doi: 10.1093/aob/mcl037 
Schinkel, C.C.-F. (2019). Reproductive strategies of alpine apomictic plants under different ecological conditions. (Doctoral dissertation, Niedersächsische Staats-und Universitätsbibliothek Göttingen)

Schinkel, C.C., Syngelaki, E., Kirchheimer, B., Dullinger, S., Klatt, S., and Hörandl, E. (2020). Epigenetic patterns and geographical parthenogenesis in the alpine plant species Ranunculus kuepferi (Ranunculaceae). International journal of molecular sciences 21(9), 3318. doi: 10.3390/ijms21093318

Schinkel, C.C.F., Kirchheimer, B., Dellinger, A.S., Klatt, S., Winkler, M., Dullinger, S., et al. (2016). Correlations of polyploidy and apomixis with elevation and associated environmental gradients in an alpine plant. $A \circ B$ Plants 8.

Schinkel, C.C.F., Kirchheimer, B., Dullinger, S., Geelen, D., De Storme, N., and Hörandl, E. (2017). Pathways to polyploidy: indications of a female triploid bridge in the alpine species Ranunculus kuepferi (Ranunculaceae). Plant Systematics and Evolution 303(8), 1093-1108. doi: 10.1007/s00606-017-1435-6.

Schlüter, P.M., and Harris, S.A. (2006). Analysis of multilocus fingerprinting data sets containing missing data. Molecular Ecology Notes 6(2), 569-572. doi: 10.1111/j.14718286.2006.01225.x.

Schmidt, A., Schmid, M.W., and Grossniklaus, U. (2015). Plant germline formation: common concepts and developmental flexibility in sexual and asexual reproduction. Development 142(2), 229-241. doi: 10.1242/dev.102103.

Schmidt, A., Schmid, M.W., Klostermeier, U.C., Qi, W., Guthörl, D., Sailer, C., et al. (2014). Apomictic and sexual germline development differ with respect to cell cycle, transcriptional, hormonal and epigenetic regulation. PLOS Genetics 10(7), e1004476. doi: 10.1371/journal.pgen.1004476.

Schoenfelder, K.P., and Fox, D.T. (2015). The expanding implications of polyploidy. The Journal of Cell Biology 209(4), 485-491. doi: 10.1083/jcb.201502016.

Schreiber, U., and Berry, J.A. (1977). Heat-induced changes of chlorophyll fluorescence in intact leaves correlated with damage of the photosynthetic apparatus. Planta 136(3), 233-238. doi: 10.1007/BF00385990

Selva, J.P., Zappacosta, D., Carballo, J., Rodrigo, J.M., Bellido, A., Gallo, C.A., et al. (2020). Genes Modulating the Increase in Sexuality in the Facultative Diplosporous Grass Eragrostis curvula under Water Stress Conditions. Genes 11(9), 969. doi: 10.3390/genes11090969.

Shah, J.N., Kirioukhova, O., Pawar, P., Tayyab, M., Mateo, J.L., and Johnston, A.J. (2016). Depletion of key meiotic genes and transcriptome-wide abiotic stress reprogramming mark early preparatory events ahead of apomeiotic transition. Frontiers in Plant Science 7(1539). doi: 10.3389/fpls.2016.01539.

Soltis, D.E., and Soltis, P.S. (1999). Polyploidy: recurrent formation and genome evolution. Trends in Ecology \& Evolution 14(9), 348-352. doi: https://doi.org/10.1016/S01695347(99)01638-9.

Soltis, D.E., Visger, C.J., and Soltis, P.S. (2014). The polyploidy revolution then... and now: Stebbins revisited. American Journal of Botany 101(7), 1057-1078.

Soltis, P.S., Marchant, D.B., Van de Peer, Y., and Soltis, D.E. (2015). Polyploidy and genome evolution in plants. Current opinion in genetics \& development 35, 119-125.

Soltis, P.S., and Soltis, D.E. (2000). The role of genetic and genomic attributes in the success of polyploids. Proceedings of the National Academy of Sciences 97(13), 7051-7057. doi: 10.1073/pnas.97.13.7051. 
Spielman, M., Vinkenoog, R., and Scott, R.J. (2003). Genetic mechanisms of apomixis. Philosophical Transactions of the Royal Society of London. Series B: Biological Sciences 358(1434), 1095-1103.

Stangherlin, A., and Reddy, A.B. (2013). Regulation of circadian clocks by redox homeostasis. Journal of Biological Chemistry 288(37), 26505-26511. doi: 10.1074/jbc.R113.457564

Stirbet, A., Lazár, D., and Kromdijk, J. (2018). Chlorophyll a fluorescence induction: can just a one-second measurement be used to quantify abiotic stress responses? Photosynthetica 56(1), 86-104. doi: 10.1007/s11099-018-0770-3.

Storchová, Z., Breneman, A., Cande, J., Dunn, J., Burbank, K., O'Toole, E., et al. (2006). Genome-wide genetic analysis of polyploidy in yeast. Nature 443(7111), 541-547. doi: 10.1038/nature05178.

Strasser, R.J., Srivastava, A., and Tsimilli-Michael, M. (2000). The fluorescence transient as a tool to characterize and screen photosynthetic samples. Probing photosynthesis: mechanisms, regulation and adaptation, 445-483. doi: 10.1016/s02697491(03)00094-0

Strasser, R.J., Tsimilli-Michael, M., and Srivastava, A. (2004). "Analysis of the chlorophyll a fluorescence transient," in Chlorophyll a fluorescence. Springer., 321-362.

Sulpice, R., Flis, A., Ivakov, A.A., Apelt, F., Krohn, N., Encke, B., et al. (2014). Arabidopsis coordinates the diurnal regulation of carbon allocation and growth across a wide range of photoperiods. Molecular Plant 7(1), 137-155. doi: 10.1093/mp/sst127

Takahashi, S., and Badger, M.R. (2011). Photoprotection in plants: a new light on photosystem II damage. Trends in Plant Science 16(1), 53-60. doi: https://doi.org/10.1016/j.tplants.2010.10.001.

Takahashi, S., and Murata, N. (2008). How do environmental stresses accelerate photoinhibition? Trends in plant science 13(4), 178-182. doi: 10.1016/j.tplants.2008.01.005

Torres, R., Romero, J., and Lagorio, M. (2021). Effects of sub-optimal illumination in plants. Comprehensive chlorophyll fluorescence analysis. Journal of Photochemistry and Photobiology B: Biology 218, 112182. doi: 10.1016/j.jphotobiol.2021.112182

Tucker, M.R., and Koltunow, A.M. (2009). Sexual and asexual (apomictic) seed development in flowering plants: molecular, morphological and evolutionary relationships. Functional Plant Biology 36(6), 490-504. doi: 10.1071/FP09078.

Ulum, F.B., Costa Castro, C., and Hörandl, E. (2020). Ploidy-dependent effects of light stress on the mode of reproduction in the Ranunculus auricomus complex (Ranunculaceae). Frontiers in Plant Science 11, 104. doi: 10.3389/fpls.2020.00104

van Baarlen, P., de Jong, H.J., and van Dijk, P.J. (2002). Comparative cyto-embryological investigations of sexual and apomictic dandelions (Taraxacum) and their apomictic hybrids. Sexual Plant Reproduction 15(1), 31-38. doi: 10.1007/s00497-002-0132-x.

Van de Peer, Y., Ashman, T.-L., Soltis, P.S., and Soltis, D.E. (2020). Polyploidy: an evolutionary and ecological force in stressful times. The Plant Cell, 11-26. doi: 10.1093/plcell/koaa015

Van de Peer, Y., Maere, S., and Meyer, A. (2009). The evolutionary significance of ancient genome duplications. Nat Rev Genet 10(10), 725-732. doi: 10.1038/nrg2600.

Van de Peer, Y., Mizrachi, E., and Marchal, K. (2017). The evolutionary significance of polyploidy. Nat Rev Genet 18(7), 411-424. doi: 10.1038/nrg.2017.26. 
Vass, I. (2012). Molecular mechanisms of photodamage in the Photosystem II complex. Biochimica et Biophysica Acta (BBA)-Bioenergetics 1817(1), 209-217. doi: 10.1016/j.bbabio.2011.04.014

Vinkenoog, R., Bushell, C., Spielman, M., Adams, S., Dickinson, H.G., and Scott, R.J. (2003). Genomic imprinting and endosperm development in flowering plants. Molecular biotechnology 25(2), 149-184.

Walker, D. (1987). The use of the oxygen electrode and fluorescence probes in simple measurements of photosynthesis. (p. 212). Sheffield: Research Institute for Photosynthesis, University of Sheffield.

Warner, D.A., and Edwards, G.E. (1993). Effects of polyploidy on photosynthesis. Photosynthesis Research 35(2), 135-147. doi: 10.1007/BF00014744

Weaver, G., and van lersel, M.W. (2020). Longer photoperiods with adaptive lighting control can improve growth of greenhouse-grown 'Little Gem'lettuce (Lactuca sativa). HortScience 55(4), 573-580. doi: 10.21273/HORTSCI14721-19.

Wei, T., Wang, Y., Xie, Z., Guo, D., Chen, C., Fan, Q., et al. (2019). Enhanced ROS scavenging and sugar accumulation contribute to drought tolerance of naturally occurring autotetraploids in Poncirus trifoliata. Plant Biotechnology Journal 17(7), 1394-1407. doi: https://doi.org/10.1111/pbi.13064.

Wu, S., Cheng, J., Xu, X., Zhang, Y., Zhao, Y., Li, H., et al. (2019). Polyploidy in invasive Solidago canadensis increased plant nitrogen uptake, and abundance and activity of microbes and nematodes in soil. Soil Biology and Biochemistry 138, 107594. doi: https://doi.org/10.1016/j.soilbio.2019.107594.

Wu, Z., Skjelvåg, A., and Baadshaug, O. (2004). Quantification of photoperiodic effects on growth of Phleum pratense. Annals of botany 94(4), 535-543. doi: 10.1093/aob/mch170

Young, B.A., Sherwood, R.T., and Bashaw, E.C. (1979). Cleared-pistil and thick-sectioning techniques for detecting aposporous apomixis in grasses. Canadian Journal of Botany 57(15), 1668-1672. doi: 10.1139/b79-204.

Yusuf, M.A., Kumar, D., Rajwanshi, R., Strasser, R.J., Tsimilli-Michael, M., and Sarin, N.B. (2010). Overexpression of $\mathrm{Y}$-tocopherol methyl transferase gene in transgenic Brassica juncea plants alleviates abiotic stress: physiological and chlorophyll a fluorescence measurements. Biochimica et Biophysica Acta (BBA)-Bioenergetics 1797(8), 1428-1438. doi: 10.1016/j.bbabio.2010.02.002.

Zappacosta, D.C., Ochogavía, A.C., Rodrigo, J.M., Romero, J.R., Meier, M.S., Garbus, I., et al. (2014). Increased apomixis expression concurrent with genetic and epigenetic variation in a newly synthesized Eragrostis curvula polyploid. Scientific Reports 4, 4423. doi: $10.1038 /$ srep04423. 


\section{List of Publications}

Ulum, F.B., Costa Castro, C., and Hörandl, E. (2020). Ploidy-dependent effects of light stress on the mode of reproduction in the Ranunculus auricomus complex (Ranunculaceae). Frontiers in Plant Science 11, 104

Ulum, F.B., Hadacek, F., and Hörandl, E. (2021). Ploidy-dependent effects of prolonged photoperiod on photosynthesis of the Ranunculus auricomus complex (Ranunculaceae). In prep

\section{Thesis declarations}

Declaration of the author's own contribution to the manuscript with multiple authors

Chapter 2:

Ulum, F.B., Costa Castro, C., and Hörandl, E. (2020). Ploidy-dependent effects of light stress on the mode of reproduction in the Ranunculus auricomus complex (Ranunculaceae). Frontiers in Plant Science 11, 104.

FU and EH designed research. FU performed research, analyzed and interpreted data. CC contributed to FCSS and microsatellite analysis. FU wrote the manuscript with the contributions of $\mathrm{EH}$.

Chapter 3:

Ulum, F.B., Hadacek, F., and Hörandl, E. (2021). Ploidy-dependent effects of prolonged photoperiod on photosynthesis of the Ranunculus auricomus complex (Ranunculaceae). In prep.

$\mathrm{EH}$ and $\mathrm{FH}$ conceived and designed the study; FU performed the photosynthesis. FU and $\mathrm{FH}$ analyzed data, and $\mathrm{FU}, \mathrm{EH}$, and $\mathrm{FH}$ wrote and consented to the manuscript 


\section{Acknowledgment}

I would like to thanks Prof. Elvira Hörandl for placing her trust in me to carry out this research, the insightful advice, and the support during the research and writing manuscript.

My thanks to Prof. Dr. Hermann Behling for very supportive of a committee member and reviewing my dissertation.

I further thank Franz Hadacek for his advice in measurement and in data analysis of photosynthesis, and contribution on the metabolite analysis for further publication.

Thank you to Camila Castro for contributing during the work of flow cytometry analysis seed screening and microsatellite analysis. Thank you to Ladislav Hodač and Salvatore Tomasello for helping during my work in molecular Lab.

The work was supported by The German Research Fund "Deutsche Forschungsgemeinschaft" DFG (project Hörandl Ho 4395 4-1) to Prof. Elvira Hörandl and my Scholarship funding was supported by Indonesia endowment fund for education (LPDP).

I express many thanks to the colleague in the Department of Systematics, Biodiversity, and Evolution of Plants, Albrecht-von-Haller Institute for Plant Sciences, University of Göttingen, for warm friendship.

Thank you also to Silvia for taking care of the plant in the climatic chamber.

My special thank you to my parents for their pray and love and to my beloved wife and my sweet kids for all love and full of happiness in accompanying me in Germany.

Finally, I would like to express my gratitude to all people that help me in many ways to complete this work. 


\section{Curriculum vitae}

Fuad Bahrul Ulum

3/2017 - 7/2021 Ph.D. Student at Albrecht-von-Haller Institute for Plant Sciences

Department of Systematics, Biodiversity and Evolution of Plants (with Herbarium), Georg-August-University Göttingen

Thesis: Ploidy-dependent effect of prolonged photoperiod to the mode of reproduction and photosynthesis of Ranunculus auricomus complex (Ranunculaceae)

11/2012 - 7/2014 Master Student of Tropical and International Forestry of Faculty of Forest Science and Forest Ecology, Georg-August-University Göttingen

Thesis: Ploidy levels and reproductive behavior in natural populations of Centotheca lappacea from Jambi, Indonesia

12/2008 - Present Lecturer at Biology Department, Faculty of mathematics and science, Jember University, Indonesia

8/2003 - 10/2007 Bachelor of Science. Department of Biology. Faculty of Mathematics and Natural Science. Jember University. Indonesia

Thesis: Production of antibiotic against Candida albicans from Actinomycetes isolates in corn extract media 